\title{
Neural dynamics of food reward : the influence of body weight, cue exposure and attention
}

Citation for published version (APA):

Frankort, A. A. C. (2013). Neural dynamics of food reward : the influence of body weight, cue exposure and attention. [Doctoral Thesis, Maastricht University]. Maastricht University. https://doi.org/10.26481/dis.20130624af

Document status and date:

Published: 01/01/2013

DOI:

10.26481/dis.20130624af

Document Version:

Publisher's PDF, also known as Version of record

\section{Please check the document version of this publication:}

- A submitted manuscript is the version of the article upon submission and before peer-review. There can be important differences between the submitted version and the official published version of record.

People interested in the research are advised to contact the author for the final version of the publication, or visit the DOI to the publisher's website.

- The final author version and the galley proof are versions of the publication after peer review.

- The final published version features the final layout of the paper including the volume, issue and page numbers.

Link to publication

\footnotetext{
General rights rights.

- You may freely distribute the URL identifying the publication in the public portal. please follow below link for the End User Agreement:

www.umlib.nl/taverne-license

Take down policy

If you believe that this document breaches copyright please contact us at:

repository@maastrichtuniversity.nl

providing details and we will investigate your claim.
}

Copyright and moral rights for the publications made accessible in the public portal are retained by the authors and/or other copyright owners and it is a condition of accessing publications that users recognise and abide by the legal requirements associated with these

- Users may download and print one copy of any publication from the public portal for the purpose of private study or research.

- You may not further distribute the material or use it for any profit-making activity or commercial gain

If the publication is distributed under the terms of Article $25 \mathrm{fa}$ of the Dutch Copyright Act, indicated by the "Taverne" license above, 
NeUral DYNAMics OF FOOD REWARD

THE INFLUENCE OF BODY WEIGHT, CUE EXPOSURE AND ATTENTION 
(C) Astrid Anna Catharina Frankort, Maastricht 2013

ISBN 978-90-9027621-2

Cover concept \& image: Astrid Frankort

Cover layout: Michał Sławiński

Layout and typesetting: * ${ }^{\star}$ studio Michał Sławiński, thesisprint.eu

All rights reserved. No parts of this thesis may be reproduced or transmitted in any form or by any means, electronic or mechanical, including photocopy, recording or any other information storage or retrieval system, without permission in writing from the author, or, when appropriate, from the publishers holding the copyright of the published articles. 


\title{
NeURAL DYNAMICS OF FOOD REWARD The Influence of BODY Weight, CUE EXPOSURE AND ATTENTION
}

\author{
Proefschrift \\ ter verkrijging van de graad van doctor aan de Universiteit Maastricht, \\ op gezag van de Rector Magnificus, Prof. dr. L.L.G. Soete, \\ volgens het besluit van het College van Decanen, \\ in het openbaar te verdedigen op maandag 24 juni 2013 om 16:00 door
} Astrid Anna Catharina Frankort 


\section{Promotor}

Prof. dr. A.T.M. Jansen

\section{Copromotoren}

Dr. A. Roefs

Dr. A. Roebroeck

\section{BeOORDELINGSCOMMISSIE}

Prof. dr. B. Jansma (voorzitter)

Prof. dr. C. de Graaf (Wageningen UR)

Dr. S. Higgs (University of Birmingham, United Kingdom)

Dr. R. Ruiter

Prof. dr. A. Schols

Dit proefschrift is tot stand gekomen met behulp van financiering door de Nederlandse Organisatie voor Wetenschappelijk Onderzoek (NWO), subsidie nummer 400.06.148 aan Prof. dr. A.T.M. Jansen. 


\section{Contents}

Chapter 1

Introduction

Chapter 2 Reward activity in satiated overweight women is decreased during unbiased viewing but increased when imagining taste: an event-related fMRI study

CHAPTER 3 Reward or inhibition when seeing palatable food?

It all depends on your weight and your focus of attention

Chapter 4

The craving stops before you feel it: neural correlates of chocolate craving during cue exposure with response prevention

Chapter 5

Neural predictors of chocolate intake

following chocolate exposure

Chapter 6 Summary and general discussion

\section{Appendix}

References

Dankwoord

Curriculum Vitae 

Chapter 1

INTRODUCTION 


\section{INTRODUCTION}

The invention of functional magnetic resonance imaging (fMRI) in 1991 (Bandettini, 2012; Belliveau et al., 1991) made it possible to study processes in the brain in a non-invasive manner. Since then, the number of studies in the field of cognitive neuroscience has increased exponentially, a growing number of which employ fMRI as a research technology (Rosen \& Savoy, 2012). This is also the case in the field of food reward and food cue processing studies, but the neural processes of normal and abnormal eating behaviour (i.e., eating disorders and obesity) are still not fully understood.

Research in this area is complicated because many factors influence eating behaviour. For example, body weight status and personal trait characteristics, like eating restraint, influence the amount and quality (e.g., high-fat, low-nutrient) of food that is eaten (Rotenberg et al., 2005). The same is true for gender (Smeets et al., 2006) and for personal state characteristics like degree of hunger, mood, sleepiness, hormone levels and stress (Asterholm \& Scherer, 2012; Hepworth, Mogg, Brignell, \& Bradley, 2010; Killgore et al., 2013; Rutters, Nieuwenhuizen, Lemmens, Born, \& Westerterp-Plantenga, 2009; Schloegl, Percik, Horstmann, Villringer, \& Stumvoll, 2011). Also food characteristics, like its propensity to be ingested more or less quickly (Higgs \& Jones, 2013; Viskaal-van Dongen, Kok, \& de Graaf, 2011) and the environment while eating are of relevance, like the presence of distractive elements (Higgs \& Woodward, 2009) and social context (Herman, Roth, \& Polivy, 2003). Furthermore, the duration during which the participant has been exposed to the food without eating it has an effect (Coelho, Nederkoorn, \& Jansen, 2013; Ferriday \& Brunstrom, 2011). These examples show that there are many influencing factors in the research of eating behaviour, which makes it difficult to establish a parsimonious model.

Within the study of neural correlates of food reward, several personal, task-related and stimulus-related factors are also of relevance. For example, trait reward sensitivity was highly correlated with activation to images of appetizing foods in a fronto-striatalamygdala-midbrain network (Beaver et al., 2006). Further, the type of attention that is paid to the food stimulus plays a large role. In a study in which participants had to process the food stimuli in different ways according to instructions (passive viewing, focus on taste, focus on health or suppression of craving), it was found that brain reward regions were more active when participants focused on taste than when they did not (Siep et al., 2012), while regions involved in executive control were most active during suppression of craving as compared to the remaining conditions (Scharmüller, Übel, Ebner, \& Schienle, 2012; Siep et al., 2012). Further influence is exerted by the characteristics of the food stimulus, like calorific value and palatability (Coletta et al., 2009; Nummenmaa et al., 2012). Finally, cue exposure affects the activation in brain regions related to reward and attention, although this was shown in studies on alcohol dependence instead of food reward (Mainz et al., 2012; Vollstädt-Klein et al., 2011). The goal of this dissertation was 
to investigate how food cues are processed in the brain and how neural activation is influenced by the calorific value and palatability of the food stimulus, attention type, body weight status, and exposure to the food (i.e., smelling the food).

The relevance of this study is not just purely scientific. The awareness that overeating and the wrong nourishment have an increasing impact on the general wellbeing and overall health of the population has increased over the last decades. Evidence of this can, for example, be found in the reports of authorities that guide policy-makers (CBS, 2012a, 2012b, 2012c; Sassi, Cecchini, \& Devaux, 2010; Sassi \& Devaux, 2012). Eating behaviour is controlled both by internal metabolic hunger signals (called homeostatic signalling) and by hedonic environmental signals in the absence of metabolic need (non-homeostatic processes), which intimately interact neurally (Berthoud, 2011). In the modern Western world, palatable high-calorie food is abundant and the hedonic 'eat more'-command is stronger than the 'stop eating'-command (Zheng, Lenard, Shin, \& Berthoud, 2009), leading to weight gain and a global increase in obesity (Swinburn et al., 2011). The investigation of the interaction between homeostatic and non-homeostatic processes in the brain is important to identify ways to intervene in case the eating behaviour becomes abnormal, as in obesity, where hedonic signals dominate (Zheng et al., 2009), or as in anorexia nervosa where excessive top-down cognitive control seems to alter striatal activity in a way that long-term goals (avoiding food, getting thin) become more important than short-term goals (eating food) (Kaye, Fudge, \& Paulus, 2009).

\section{CUE REACTIVITY AND NEURAL CORRELATES OF FOOD REWARD AND INHIBITION}

Living beings depend on food for survival. Therefore food is a powerful primary reinforcer (Berridge, 1996, 2004; Epstein \& Leddy, 2006) and food cues elicit reactivity like insulin release and increased salivation (Jansen, Havermans, \& Nederkoorn, 2011). These physiological responses are subjectively experienced as craving. In overweight people, cue reactivity is increased compared with healthy-weight people (Ferriday \& Brunstrom, 2011; Jansen et al., 2003). On a neural level, reactivity to food cues has been associated with a heightened activation in several brain regions: the amygdala, corpus striatum (nucleus accumbens, caudate, putamen and globus pallidus), insula, hippocampus, substantia nigra and ventral tegmental area as well as anterior cingulate, prefrontal, posterior fusiform and orbitofrontal cortices (for reviews see: García-García et al., 2013; Kringelbach, 2009; Rolls, 2011; Van der Laan et al., 2011). The activation of these regions has been found to correlate with the reward value of a food stimulus: the more rewarding the stimulus in terms of subjective liking, the more active these regions were. An increased activation of these regions in response to high-calorie versus neutral or low-calorie pictures has been 
associated with overweight (e.g., McCaffery et al., 2009; Murdaugh, Cox, Cook, \& Weller, 2012; Rothemund et al., 2007; Scharmüller et al., 2012; Stice, Spoor, Bohon, Veldhuizen, \& Small, 2008; Stoeckel et al., 2008; Wallner-Liebmann et al., 2010).

The neural processing of food cues is not only accompanied by activity in reward regions, but often also by activity in inhibitory or executive control regions: subdivisions of the prefrontal cortex (PFC) like medial, ventrolateral and dorsolateral PFC, as well as posterior parietal cortex (Kishinevsky et al., 2012; Stoeckel, Murdaugh, Cox, Cook, \& Weller, 2012; Yokum, Ng, \& Stice, 2011a). The more executive control is exerted, for example to suppress craving for a food stimulus, the more activation these regions will show (Hutcherson, Plassmann, Gross, \& Rangel, 2012; Kober et al., 2010; Scharmüller et al., 2012; Siep et al., 2012). In its turn, body mass index (BMI) influences the amount of executive control. BMI has been shown to correlate inversely with activation of frontal inhibitory regions when participants were instructed to inhibit their responses to appetizing food pictures in a go/no-go task (Batterink, Yokum, \& Stice, 2010): the higher the $\mathrm{BMI}$, the less inhibition to appetizing foods was exerted. In addition in a positron emission tomography (PET) experiment an association was found between a higher BMI and a lower baseline metabolism in prefrontal regions (Volkow et al., 2009).

Recent studies have shown that activation of brain regions in response to food cues is not just a symptom of (ab)normal eating behaviour, it can even predict subsequent snacking behaviour and future weight change. For example, nucleus accumbens activation in response to food cues has been found to be related to subsequent snack food consumption (Lawrence, Hinton, Parkinson, \& Lawrence, 2012) and to weight gain after six months (Demos, Heatherton, \& Kelley, 2012). Similarly, participants with a higher response in brain reward regions to high-calorie food cues gained more weight in one year than participants with a lower response in these brain regions (Stice, Yokum, Bohon, Marti, \& Smolen, 2010; Yokum, Ng, \& Stice, 2011b) and were less successful in losing weight in a weight-loss program after the scanning session (Murdaugh et al., 2012). In a delay discounting task measuring brain activation in response to difficult versus easy delay discounting trials, less activation in executive control regions was also predictive of more weight gain over the subsequent years (Kishinevsky et al., 2012).

\section{THE INFLUENCE OF ATTENTION AND WEIGHT STATUS ON THE NEURAL PROCESSING OF FOOD STIMULI}

The environmental context and the type of attention which is voluntarily or involuntarily directed to food cues, like focus on taste or focus on health, influences the way in which these food cues are perceived. For example, in an experiment in which food preference was measured using an implicit measure of association (Roefs et al., 2011), it was found that not weight-status but current focus of attention (focus on palatability or focus on 
health) determined the response on the implicit measure of food preference. Selective attention enhanced processing of relevant information and suppressed processing of stimuli that had to be ignored (Mack \& Rock, 1998). The attention that is paid to a stimulus also influences the way in which this stimulus is processed in the brain. For example, activation in the amygdala depended on the focus of attention during cognitive modulation (Pessoa, Padmala, \& Morland, 2005). In this study, while showing pictures of a face with a bar at the right and left side of the face, amygdala activation was stronger when the focus of attention was on the faces (because the instruction was to determine the gender of the face shown), as compared to the condition when the focus of attention was on the orientation of the bars next to the face (because the instruction was to determine if the bars had the same orientation). This suggests that the focus of attention has an effect on brain activation, even if the same stimuli are shown. In another study, the fusiform cortex was more active when faces were presented in task-relevant as compared to task-irrelevant positions (Vuilleumier, Richardson, Armony, Driver, \& Dolan, 2004), which shows that the type of attention involuntarily paid to the faces influenced the amount of brain activation. As a final example, repetition-related decreases in fMRI responses were different for previously attended versus ignored items (Vuilleumier, Schwartz, Duhoux, Dolan, $\&$ Driver, 2005), suggesting that the amount of attention previously paid to a stimulus influences brain activation in response to its renewed presentation.

Quite surprisingly, many studies on food cue processing in the brain do not take the role of attention paid to these cues into account. The majority of studies employ only a condition of passive viewing (e.g., Bruce et al., 2010; Coletta et al., 2009; Ho, Kennedy, \& Dimitropoulos, 2012; Killgore et al., 2003; Martens et al., 2013; Martin et al., 2010; Murdaugh et al., 2012; Rothemund et al., 2007; Schienle, Schäfer, Hermann, \& Vaitl, 2009; Simmons, Martin, \& Barsalou, 2005; Stoeckel et al., 2008). Therefore, we wanted to investigate how different types of attention influenced the processing of food stimuli in both people with and without overweight (Chapter 2). The first type of attention was unbiased viewing without prior instructions. This means that participants were not biased or instructed on how they should perceive the presented food stimuli. The second attention type was taste imagination, during which participants were asked to imagine the taste of the presented food stimuli. The focus of the analysis in Chapter 2 was on the difference between overweight and healthy-weight people regarding the response to high-calorie palatable food stimuli during the two different types of attention. It was expected that overweight participants would have more reward-related brain activity than healthy-weight participants when viewing high-calorie palatable food stimuli. This group difference was expected to be even more pronounced in the unbiased viewing condition than in the condition of taste imagination, because in the taste imagination condition all participants would be engaged in the evaluation of food palatability, thereby reducing possible group differences. For a detailed explanation of this experiment and of the first analysis of these data, the reader is referred to Chapter 2. 
In the study described in Chapter 2, food stimuli that ranged from unpalatable to very palatable were presented to both healthy and overweight participants. Previous research suggests that a variation in palatability may have different effects on food intake in overweight and healthy-weight participants, in that obese participants seem to be more finicky with regard to their food consumption than healthy-weight participants (Pliner, Herman, \& Polivy, 1990). Surprisingly, this finickiness is not mirrored by subjective reports of liking of food items, because these do not differ between people with and without overweight (Dimitropoulos, Tkach, Ho, \& Kennedy, 2012; Frankort et al., 2012; Nummenmaa et al., 2012; Rothemund et al., 2007; Scharmüller et al., 2012). Considering that self-reports can be distorted by an insufficient introspection (Nisbett \& Wilson, 1977) or by socially desirable answering tendencies (Schwarz \& Oyserman, 2001), it can be revealing to directly study brain-reward processes in response to palatable and less palatable food cues. Neural activation can be a better predictor of behaviour than subjective reports (Falk, Berkman, Mann, Harrison, \& Lieberman, 2010; Falk, Berkman, Whalen, \& Lieberman, 2011; Grüsser et al., 2004; Kosten et al., 2006). It was therefore expected that the finickiness of obese as compared to healthy-weight participants would be represented by a difference in neural reward processing of the food stimuli.

To this end, the data of the first study (see Chapter 2) were re-analysed (Chapter 3). The focus of this re-analysis was on the difference between overweight and healthyweight participants in response to palatable versus neutral food stimuli, collapsed over calorific value of the food stimuli. It was hypothesised that the overweight participants would have a stronger activation in response to palatable versus neutral food stimuli in regions associated with reward than the healthy-weight participants, especially during the condition of taste imagination. The analyses were different from the ones conducted in Chapter 2, because additionally to the high-calorie palatable stimuli, both stimuli regarded as neutral and low-calorie stimuli were taken into account in the new analyses. Further, the analyses did not focus on an interaction between group and the attention condition, but on an interaction between group and palatability.

\section{THE INFLUENCE OF CUE EXPOSURE ON CUE REACTIVITY}

Food cue reactivity has been shown to be greater in overweight adults, overweight children and in binge eaters compared with healthy-weight participants (Ferriday \& Brunstrom, 2011; Jansen et al., 2003; Sobik, Hutchison, \& Craighead, 2005). Formerly obese successful dieters were found to have decreased cue reactivity as compared to currently obese unsuccessful dieters (Jansen, Stegerman, Roefs, Nederkoorn, \& Havermans, 2010). Neuroimaging studies showed that a higher activation of brain reward regions in response to food cues which usually elicit cue reactivity (i.e., high-calorie food pictures) versus control pictures was predictive of less success in losing weight (Murdaugh et al., 
2012) or even of weight gain (Demos et al., 2012). These findings suggest that increased cue reactivity may lead to weight gain, and decreased cue reactivity may lead to weight loss or maintenance of weight loss. Therefore, if one wants to increase the likelihood of an ultimately successful weight-loss, the question of how to decrease cue reactivity arises.

One possibility to decrease cue reactivity is to use cue exposure with response prevention (CERP). During CERP the participant is exposed to food cues or contexts that normally predict food intake, but is not allowed to eat. This exposure continues for about an hour, or as long as necessary to extinguish the urge to eat. Because eating is not allowed, the association of the food cue with food intake is disrupted. The theory behind this is that cue reactivity is classically conditioned (Jansen, 1998): it has been learned that the presence of a certain food cue (conditioned stimulus, CS) predicts intake of that food (unconditioned stimulus, US), just like the dogs in Pavlov's famous experiments learned that the ringing of the bell (CS) predicted feeding (US) (Pavlov, 1927). By not allowing the participant to eat, the association between CS and US is disrupted. After several sessions of CERP in different contexts, the food cue no longer predicts intake and the conditioned response (cue reactivity and craving) will be extinguished (Van Gucht et al., 2008; Jansen, Broekmate, \& Heymans, 1992; Jansen, Van den Hout, De Loof, Zandbergen, \& Griez, 1989; Martinez-Mallén et al., 2007; Toro et al., 2003).

In the study presented in Chapter 4, we were interested in the effects of CERP on a neural level over the course of time in healthy-weight people. As a food cue, we chose the food most frequently craved by Western women: chocolate (Pelchat, 1997). After the exposure session, chocolate intake was measured in a bogus chocolate taste test. A control manipulation was done in a different group of participants with a pencil instead of chocolate. We expected that in the exposure group, chocolate craving would go up after the beginning of the session, would peak in the middle of the session and from then on would decrease and extinguish. This expected increase, peak and decrease of craving was hypothesised to be mirrored by activation in brain reward regions in the exposure group as compared to the control group. Further details of this experiment are described in Chapter 4, which focuses on the course of craving during the cue exposure session and the corresponding brain reward activation.

In the study described in Chapter 5, it was examined if CERP influenced subsequent chocolate intake and if brain activation could predict subsequent chocolate intake. Previous studies showed that in the long run, the activation of specific brain regions can be predictive of weight gain or weight loss (Demos et al., 2012; Kishinevsky et al., 2012; Murdaugh et al., 2012; Stice et al., 2010; Yokum et al., 2011b). The prediction of shortterm intake, however, has been investigated so far in only one study (Lawrence et al., 2012). Therefore, in Chapter 5 we investigated if brain activation in response to chocolate versus control pictures explained additional variance in eating behaviour in the exposure and in the control group, above and beyond the variance already explained by selfreported craving. There is reason to believe that this could be the case based on previous 
research in which brain activation was a better predictor of subsequent behaviour than self-reported craving, intentions, or attitudes (Falk et al., 2010, 2011; Grüsser et al., 2004; Kosten et al., 2006).

\section{IN SUMMARY}

Summarizing, it can be said that the experiments and studies described in this dissertation dealt with the question of how food cues of varying palatability and of high and low calorific value are processed in the brain in overweight and healthy-weight participants. Furthermore the influence that is exerted by the type of attention on the neural processing of food cues was investigated, like unbiased viewing without prior instructions or focus on taste. Additionally, we looked at the effect of cue exposure with response prevention on subjective craving for chocolate and on neural responses to chocolate pictures versus neutral pictures over the course of a session of one hour. Finally, the predictive characteristics of both subjective craving and brain activation on subsequent chocolate intake were examined. Chapter 6 contains a summary and general discussion of these studies and provides an outlook regarding future studies and possible clinical implications. 


\title{
Chapter 2
}

\author{
REWARD ACTIVITY IN SATIATED OVERWEIGHT \\ WOMEN IS DECREASED DURING UNBIASED VIEWING \\ BUT INCREASED WHEN IMAGINING TASTE: \\ AN EVENT-RELATED FMRI STUDY
}

Frankort, A., Roefs, A., Siep, N., Roebroeck, A., Havermans, R., \& Jansen, A. (2012). Reward activity in satiated overweight women is decreased during unbiased viewing but increased when imagining taste: an event-related fMRI study. International journal of obesity, 36(5), 627-37. doi:10.1038/ijo.2011.213 


\section{Abstract}

Objective: The aim of this fMRI study was to investigate reward-related brain activity in satiated overweight and healthy-weight participants in response to high-calorie palatable food pictures, when viewing the pictures without prior instructions (called unbiased viewing) versus imagining the taste of the shown pictures (called taste imagination). We predicted that neural activation in brain reward regions would be greater in overweight participants than in healthy-weight ones and that this difference between groups would be strongest during unbiased viewing.

Method: Neural activation was measured using fMRI in 14 overweight (mean BMI: 29.8) and 15 healthy-weight (mean BMI: 21.1) participants who were satiated, in response to palatable and unpalatable high-calorie and low-calorie food pictures, presented in an eventrelated design during two conditions: unbiased viewing (no prior instructions) versus taste imagination.

Results: A group $\times$ condition interaction was found in 14 brain regions involved in food reward processing, during the presentation of highcalorie palatable food stimuli. During the taste imagination condition, neural activation in these regions was greater in the overweight participants than in the healthy-weight ones. Contrary to our expectations, the opposite pattern was observed during unbiased viewing: activation in reward regions in the overweight participants was reduced compared to the healthy-weight ones. In all brain reward regions except for the left amygdala, the group $\times$ condition interaction was specific to high-calorie palatable food stimuli.

Conclusion: Greater reward activity in the overweight participants compared to the control group when imagining taste may represent an increased reward response induced by high-calorie palatable food. During unbiased viewing, reduced reward activation in the overweight participants compared to those with a healthy weight may reflect avoidance of high-calorie palatable food stimuli. Taken together, this pattern of activation may reflect ambivalence in the overweight group between desire for (in the taste imagination condition) and avoidance of (in the unbiased viewing condition) high-calorie palatable food stimuli. 


\section{INTRODUCTION}

Energy intake exceeding energy expenditure results in weight gain and overweight when this imbalance is enduring (Epstein, Leddy, Temple, \& Faith, 2007; J. O. Hill, Catenacci, \& Wyatt, 2005; Schoeller, 2009). The high incidence of obesity (Berghöfer et al., 2008; Kelly, Yang, Chen, Reynolds, \& He, 2008; World Health Organization, 2007) has been linked to the consumption of energy-dense palatable foods (Drewnowski, 2007; McCrory et al., 1999; B. J. Rolls, 2000). Overweight people may consume more of these foods because they are more reinforcing to them, as suggested by studies finding that overweight and obese people work harder to obtain high-calorie palatable foods than do lean control participants (Epstein, Temple, et al., 2007; Giesen, Havermans, Douven, Tekelenburg, \& Jansen, 2010). Moreover, it has been shown that overweight participants are more sensitive to reward (Davis et al., 2007; Franken \& Muris, 2005) than healthy-weight ones. The goal of the current study is to further investigate the neural correlates of high-calorie food-reward processing in overweight and healthy-weight participants.

Brain regions involved in the processing of food reward are the amygdala, hippocampus, ventral pallidum, nucleus accumbens and striatum, the ventral tegmental area and substantia nigra, as well as the anterior cingulate, orbitofrontal, insular, posterior fusiform, dorsolateral prefrontal, and medial prefrontal cortices (Berthoud, Lenard, \& Shin, 2011; Kringelbach, 2009; Van der Laan et al., 2011). Previous neuroimaging research in obese versus healthy-weight participants showed increased activity in several of these brain reward regions while viewing high-calorie versus neutral or low-calorie food pictures in a hungry state (Stoeckel et al., 2008), in a non-hungry state (Rothemund et al., 2007; Wallner-Liebmann et al., 2010), and in response to anticipated or actual consumption of a chocolate milkshake in a hungry state (Stice et al., 2008). Most of these studies involved 'unbiased viewing', meaning that food pictures are shown without the instruction to imagine their taste.

In addition to an elevated brain response to food stimuli, compared to healthyweight controls an abnormal connectivity between regions of the reward network in obese participants has been reported, with some of these regions showing an excessive and some showing a deficient interaction (Stoeckel et al., 2009). According to the authors, this may explain the differences in drive to consume foods. In addition, activation of frontal inhibitory regions has been shown to correlate inversely with body mass index (BMI) when participants were instructed to inhibit their responses to appetizing food pictures in a go/no-go task (Batterink et al., 2010). All in all these studies show that overweight women exhibit a greater response in reward-related regions in response to food cues than healthy-weight women.

Food reward processing is influenced not only by calorific value and palatability of food stimuli, but also by attention. For food-reward-related activity to occur in lean individuals, attention to food seems to be necessary, more specifically: the evaluation or 
imagination of its taste. This was shown by an earlier study from our laboratory (Siep et al., 2009) in healthy-weight participants after food deprivation and during satiation; this study found that the OFC and the amygdala, both involved in encoding the predictive reward value of stimuli (Arana et al., 2003; Gottfried, O’Doherty, \& Dolan, 2003), were activated only during taste imagination of high-calorie and low-calorie food pictures and not when judging the orientation of bars presented together with the foods. Likewise, activation of the left lateral OFC in healthy participants depended on whether or not a taste was explicitly evaluated (Bender, Veldhuizen, Meltzer, Gitelman, \& Small, 2009).

Direct comparisons of food reward processing between overweight participants and healthy-weight ones during different conditions of attention have not yet been conducted. We predict that overweight participants will pay more attention to the palatability of food than healthy-weight controls during unbiased viewing (i.e. viewing without any prior instructions) and it is expected that this is reflected in increased reward-related brain activity. This group difference may be less pronounced when imagining the taste of the food presented in the pictures, since all participants are then evaluating food palatability and may thus show resembling brain reward activity. The current study tests this hypothesis by comparing overweight and healthy-weight participants during unbiased viewing versus a condition where they imagine the taste of the food shown in pictures (in the following called taste imagination).

The participants in this study were tested in a state of satiation. Although the majority of studies investigating food reward processing differences between obese versus healthy-weight participants have been conducted during hunger or moderate deprivation (Batterink et al., 2010; Cornier et al., 2009; Stice et al., 2008; Stoeckel et al., 2008, 2009), the excess of energy intake in obesity is at least partly due to eating in the absence of hunger, also called non-homeostatic eating (Fisher \& Birch, 2002; Zheng et al., 2009). Therefore, the current study seeks to investigate food reward-processing differences between overweight participants and healthy-weight ones while they are satiated. It is hypothesised that overweight participants show more reward-related activity in response to high-calorie palatable food stimuli than healthy-weight participants. This difference between the groups will be stronger in the unbiased condition than in the taste imagination condition. Furthermore, no such difference is expected for low-calorie or unpalatable food.

\section{Method}

\section{Participants}

Participants were 14 overweight (BMI $>25$, range current study: $27 \leq \mathrm{BMI} \leq 38)$ and 15 healthy-weight $(18 \leq \mathrm{BMI} \leq 25$, range current study: $19 \leq \mathrm{BMI} \leq 23)$ Dutch females of Caucasian ethnicity, who were recruited both in the general population by an advertise- 
ment in a local newspaper and among students and employees from Maastricht University, and were matched on education level. In the overweight group, 4 participants were obese $(\mathrm{BMI} \geq 30)$. Due to problems with the scanner coil, one additional overweight participant was excluded from analyses. There were no group differences regarding age, hunger, positive and negative affect. The overweight participants reported to be more restrained in their eating than the healthy-weight participants. See Table 1 for participant characteristics. In addition to the usual fMRI exclusion criteria (e.g., metal implants), exclusion criteria were a self-reported mental disorder history or family history of eating disorders. Also vegetarian, left-handed or women currently on a weight-loss diet were excluded from participation. Finally, as the menstrual cycle phase modulates reward-related and arousal-related brain activation in women (Dreher et al., 2007) as well as food intake (Bryant, Truesdale, \& Dye, 2006), participants were selected on the basis of a relatively stable hormonal balance, which is created by the use of a one-phase combined oral contraceptive (Guillebaud, 1987). Participants received course credits or $€ 30$ for their participation.

Table 1. Participant characteristics

\begin{tabular}{|c|c|c|c|c|c|c|}
\hline & \multicolumn{2}{|c|}{$\begin{array}{l}\text { Healthy-weight } \\
\qquad(\mathrm{n}=15)\end{array}$} & \multicolumn{2}{|c|}{$\begin{array}{c}\text { Overweight } \\
(\mathrm{n}=14, \text { of which } 4 \text { obese })\end{array}$} & \multirow[b]{2}{*}{$t(27)$} & \multirow[b]{2}{*}{$p$} \\
\hline & $M$ & $S D$ & $M$ & $S D$ & & \\
\hline Age (yrs) & 23.1 & 5.4 & 23.9 & 7.6 & 0.35 & 0.73 \\
\hline BMI & 21.1 & 1.1 & 29.8 & 3.3 & 9.43 & $<.001$ \\
\hline $\begin{array}{l}\text { Time between lunch } \\
\text { and scan (hrs) }\end{array}$ & $01: 24$ & $00: 12$ & $01: 32$ & $00: 28$ & 0.92 & 0.37 \\
\hline Hunger $^{1}$ & 20.8 & 8.7 & 17.9 & 9.9 & 0.84 & 0.41 \\
\hline Positive affect ${ }^{2}$ & 28.3 & 5.9 & 28.3 & 4.4 & 0.02 & 0.98 \\
\hline Negative affect ${ }^{2}$ & 11.5 & 2.4 & 12.4 & 2.5 & 0.98 & 0.34 \\
\hline Restraint $^{3}$ & 8.8 & 3.1 & 13.9 & 3.2 & 4.35 & $<.001$ \\
\hline
\end{tabular}

Abbreviations. $\mathrm{BMI}=$ Body Mass Index.

${ }^{1}$ Average score of four $100 \mathrm{~mm}$ VAS scales (hunger, fullness, desire to eat, and amount of food that could currently be eaten), ranging from 0 to 100, with a higher score representing a higher level of hunger. One of the subscales (fullness) was likely filled out inversely by seven participants and scores were corrected; however, correction did not affect group similarity (before correction, mean hunger ratings were $23.2 \pm 9.8$ (healthy-weight) and $22.8 \pm 12.6$ (overweight), $t(27)=0.10, p=0.92$ ).

${ }^{2}$ Scored on 5-point Likert scales, with a total score ranging from 10 (very little) to 50 (very strong).

${ }^{3}$ Scored on the Restraint Scale (Herman \& Polivy, 1980), with a minimum total score of 0 (no restraint) and a maximum of 35 (high restraint). 


\section{Stimuli}

To assess if responses were specific to high-calorie palatable food we also included pictures of unpalatable and low-calorie items. Pictures of 16 different food items were purchased online (www.istockphoto.com). Each item, represented by two different pictures, belonged to one of four categories: items considered delicious according to a pilot study (described below), either high-calorie (chocolate, croissant, French fries, and ice cream) or low-calorie (strawberries, grapes, melon, and popcorn), and items considered distasteful, also high-calorie (herring, paté, peanut butter, and walnuts) or low-calorie (radishes, Brussels sprouts, chicory, and sauerkraut).

The selection of food stimuli was based on a pilot study, which included 18 female Dutch students of Maastricht University who did not participate in the current fMRI study. The food pictures were rated on palatability and calorific value on visual analogue scales (VAS: 0 - 100). The aim of the selection procedure was to obtain a set of stimuli in which every participant in the scanner study would find highly palatable as well as unpalatable stimuli, of high and of low calorific value.

\section{Assessment}

BMI. Participant's weight and height were measured to determine BMI $\left[\mathrm{kg} / \mathrm{m}^{2}\right]$.

Hunger. Hunger was measured with four VAS items (0 to 100) of a hunger questionnaire (Friedman, Ulrich, \& Mattes, 1999). The total score is calculated by averaging these VAS ratings and can range from 0 'not hungry at all' to 100 'very hungry'. VAS about hunger, appetite and satiety are tolerably valid and reliable (Stubbs et al., 2000).

Restraint Scale. Validity studies (Van Strien, Herman, Engels, Larsen, \& Van Leeuwe, 2007; Williamson et al., 2007) have shown that the Restraint Scale (Herman \& Polivy, 1980) measures the extent to which the participant intends to restrain her food intake, shows concerns about her body weight, and is susceptible to overeating. The minimum total score is 0 and the maximum is 35 . Higher scores reflect more intentions to restrain food intake. The Restraint Scale is sufficiently valid and reliable (Scagliusi et al., 2005; Van Strien et al., 2007; Williamson et al., 2007).

Positive and Negative Affect Schedule (PANAS). The state version of the PANAS (Watson, Clark, \& Tellegen, 1988) was used measuring current positive and negative affect. Both subscales consist of 10 items each, which are rated from 1 (very little or not at all) to 5 (very strong). Subscale item scores are summed to obtain positive and negative affect scores. Higher scores reflect increased positive or negative affect. The PANAS scales are internally consistent, reliable and valid (Watson et al., 1988). This questionnaire was assessed to evaluate mood differences between groups, as there is evidence that mood influences the reaction to food pictures (Killgore \& Yurgelun-Todd, 2006). 
Palatability ranking of food items. All food items were ranked for palatability, separately for high-calorie and low-calorie items. All analyses used individual rankings.

Rating of stimuli. All stimuli were rated on palatability and calorific value using $100 \mathrm{~mm}$ VASs.

\section{Experimental Design and Task}

The experimental design was a mixed 2 (BMI: healthy-weight vs. overweight) $\times 2$ (calorific value: high vs. low $) \times 2$ (palatability: palatable vs. unpalatable) $\times 2$ (attention type: unbiased viewing vs. taste imagination) design, with repeated measures on the factors calorific value, palatability and attention type.

\section{Stimulation Protocol}

The stimuli were presented in a fast event-related manner in cycles of eight stimuli: two food items for each of the four categories (see Figure 1). At the end of each cycle, a screen appeared with an overview of the preceding eight pictures. The participant was asked to choose three products. In the unbiased viewing condition (runs 1 and 2) no further instructions were provided for the task. In the taste imagination condition (runs 3 and 4), the participant was asked to pay attention to the taste of the food stimuli shown during stimulus presentation and to select three food stimuli they found most palatable during the overview screen of the preceding eight pictures. Food item selection was done by means of two button boxes, one for each hand. The runs with the unbiased viewing condition always preceded the runs with the taste imagination condition, as it is conceivable that the responses during unbiased viewing would be influenced if they would succeed the taste imagination condition.

Each stimulus was shown for $2000 \mathrm{~ms}$ after a variable inter-stimulus interval (ISI) of 2, 4, 6, or $8 \mathrm{~s}$. During this ISI a small fixation cross was presented on a black background. Stimuli were presented randomly without replacement, so that each of the 16 different food items appeared once every two cycles. As each of the four runs consisted of eight cycles, every item was shown four times in a single run and was preceded by a different ISI before every presentation (Serences, 2004). The duration of a run was approximately 12 minutes and varied due to the time needed for item selection from the overview screen. Presentation of the stimuli and recording of responses was controlled by E-Prime (Psychology Software Tools Inc., Pittsburgh, USA).

\section{Procedure}

Each participant did one fMRI session with one anatomical and four functional runs. For all participants the scanning was scheduled in the early afternoon. Each participant was requested to consume a regular lunch 1 to 1.5 hours before entering the scanner. Subjec- 


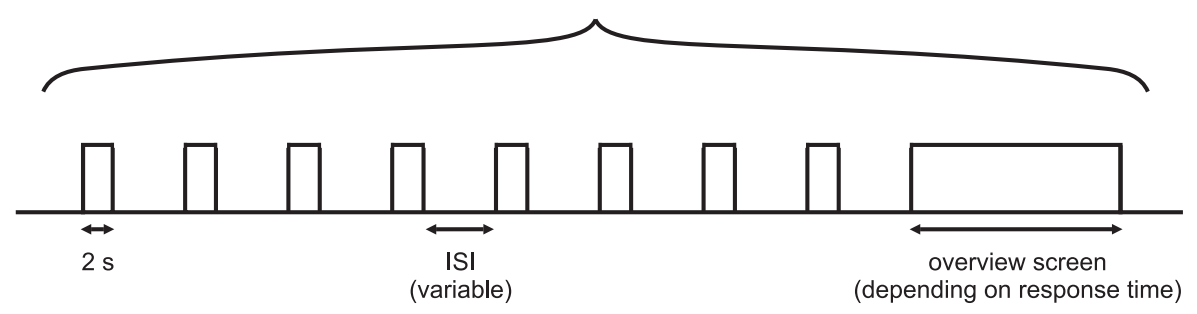

Figure 1. Graphical outline of one cycle of the fast event-related stimulation protocol, consisting of eight stimuli (half of which were low-calorie and half were high-calorie) and an overview screen. This cycle was repeated eight times within one run. Stimuli were presented during $2 \mathrm{~s}$ each. The variable inter-stimulus interval (ISI) lasted 2, 4, 6, or $8 \mathrm{~s}$, during which a small fixation cross was shown on a black background. In the overview screen, participants had to choose three of the preceding eight stimuli.

tive hunger ratings and affect were assessed before the start of the scanning session. Additionally, the participant's weight and height were measured and she was asked to write down what she ate for lunch and at what time. After entering the scanner and during the setup of the scanner sequences, the participant was given a task with neutral non-food stimuli to practise the use of the two button boxes and the selection of stimuli. Then the first two functional runs (unbiased viewing) were carried out, followed by an anatomical run and the last two functional runs (taste imagination).

After the fMRI session, the participant indicated whether she recognised the stimuli used in the scanner task. All participants recognised at least 14 different food items. Then stimuli were ranked for palatability (separately for high-calorie and low-calorie foods) and rated on palatability and calorific value. Thereafter, the Restraint Scale (Herman \& Polivy, 1980) was completed. Finally in an exit questionnaire the participant was asked whether she had perceived anything unusual about the tasks, the tests or the scanning session. No noticeable occurrences were reported. Debriefing was done by email at the end of the study. The study was approved by the Ethical Committee of the Faculty of Psychology and Neuroscience of Maastricht University.

\section{fMRI Data Acquisition}

Functional and anatomical images were acquired with a 3T Siemens Magnetom Allegra Head-only scanner. EPI parameters were optimised to reduce susceptibility and distortion artefacts in the OFC (Deichmann, Gottfried, Hutton, \& Turner, 2003; Weiskopf, Hutton, Josephs, Turner, \& Deichmann, 2007). These settings included a TE of $25 \mathrm{~ms}$, axial slices with a negative tilt angle of $30^{\circ}$, and a voxel size of $2 \times 2 \times 2.5 \mathrm{~mm}^{3}$. Further, we used parameters of TR $=2000 \mathrm{~ms}$ over 33 slices with a distance factor of $40 \%$, flip angle $90^{\circ}$, FoV $256 \times 192 \mathrm{~mm}^{2}$, imaging bandwidth $2790 \mathrm{~Hz}$ over FoV, and echo spacing 0.42 
ms, resulting in $\mathrm{T} 2{ }^{\star}$-weighted images. Each participant underwent four functional runs. The number of volumes per run varied between 338 and 369 volumes, due to differences in time taken for the choice of three products in the overview screens. For co-registration, a high-resolution T1-weighted anatomical scan was acquired after the second functional run, using an optimised MP RAGE sequence (Deichmann, Good, Josephs, Ashburner, \& Turner, 2000; Mugler \& Brookeman, 1990) with the following settings: TR $=2250 \mathrm{~ms}$, $\mathrm{TE}=2.6 \mathrm{~ms}$, flip angle $=9^{\circ}$, voxel size $1 \times 1 \times 1 \mathrm{~mm}^{3}$.

\section{Data Preprocessing}

The fMRI data were analysed with BrainVoyager QX (Brain Innovation, Maastricht, The Netherlands). The first two volumes of each functional run were discarded for analysis, due to T1 saturation effects. Preprocessing included slice scan time correction with cubic spline interpolation, three-dimensional motion correction using trilinear (for alignment) and sinc (for final resampling) interpolation, high-pass temporal filtering for removal of low-frequency noise with less than two cycles in a single run time-course (between 0.0027 and $0.0030 \mathrm{~Hz}$ cut-off, depending on the run length), and a separate mean intensity adjustment. In the data analysis following preprocessing, the motion correction and mean intensity adjustment parameters were included as predictors in the general linear model (GLM). In a total of 16 runs ( 9 in healthy-weight, 7 in overweight), the mutual collinearity of the predictors was higher than 0.9 ; here the highest correlating predictor was omitted and a collinearity lower than 0.9 was attained. After preprocessing, the functional data were co-registered with the anatomical scan, and per run a co-registered volume-time-course (VTC) was created with a voxel resolution of $2 \times 2 \times 2 \mathrm{~mm}^{3}$, representing three-dimensional space data over the course of time. Spatial normalization was performed using Talairach transformation procedures (Talairach \& Tournoux, 1988). Finally, in order to increase the signal-to-noise ratio, the VTCs were spatially smoothed with a $6 \mathrm{~mm}$ full-width-at-half-maximum isotropic Gaussian Kernel.

\section{Data Analysis}

To account for individual taste preferences, brain responses to the three most palatable and the three most unpalatable stimuli, as indicated by the individual rankings for both the high-calorie and low-calorie stimuli, were analysed for each participant. Confound predictors were set for the remaining four food items, including unrecognised stimuli, and for the overview screen. All relevant protocol conditions were convolved with a standard hemodynamic response function (Friston et al., 1998) to create the GLM design matrix. Additionally, motion and mean intensity predictors of no interest (confounds) were added. Based on this modelling approach, the baseline estimation rests on remaining fixation times during each ISI. 
Brain responses to palatable high-calorie foods were then analysed in a 2 (group) $\times 2$ (attention type) random-effects (RFX) ANOVA. Similar ANOVAs were conducted for unpalatable high-calorie foods (specificity for palatable items) and for palatable low-calorie foods (specificity for high-calorie items).

In keeping with our hypothesis, stating that the difference between groups would manifest itself mainly in the unbiased viewing condition, the resulting whole-brain statistical $F$ maps were used to define functional regions of interest (fROIs) exhibiting an interaction between BMI and attention type. For a cluster to be considered an fROI, it had to consist of at least eight contiguous voxels, each with a $p$ value of interaction below 0.01 (uncorrected). This minimal cluster size threshold was determined with the cluster-level statistical threshold estimator tool in BrainVoyager QX that implements a Monte Carlo simulation based approach of cluster-level correction of multiple comparisons (Forman et al., 1995; Goebel, Esposito, \& Formisano, 2006). Consequently, at the uncorrected level of 0.01 , only fROIs with a size larger than $64 \mathrm{~mm}^{3}$ were considered a result. The Talairach Client (www.talairach.org) was consulted to obtain an indication of the anatomical localization of the fROIs (Lancaster et al., 2000).

As our hypothesis concerned regions related to the reward system, the subsequent second-level analysis only incorporated fROIs related to the reward system and associated control regions that were mentioned in the introduction. This second-level analysis consisted of $t$ tests on average beta values per fROI per condition and aimed to find significant differences between the groups, for each attention type separately. To check for specificity and for overlapping regions, fROIs in response to high-calorie palatable food stimuli were compared to fROIs in response to low-calorie palatable or high-calorie unpalatable food stimuli.

\section{RESULTS}

\section{Stimuli}

The suitability of the stimuli was confirmed both by calorific value and palatability ratings of participants of the current study after the scanning session (see Table 2). For each participant the three food items that she ranked as most or least palatable for both calorie categories were selected for the analyses. The food stimuli in the midrange were not used in the analyses to obtain a maximum distinction between palatable and unpalatable food items.

These palatability and calorific value ratings were analyzed in two 2 (BMI: healthyweight vs. overweight) $\times 2$ (calorific value: high vs. low) $\times 2$ (palatability: ranked as least palatable vs. ranked as most palatable) ANOVAs. The palatability ratings showed a significant main effect of palatability, $F(1,27)=138.78, p<.001$, that was not moderated by group, 
Table 2. Average ratings of stimuli by healthy-weight and overweight participants

\begin{tabular}{|c|c|c|c|c|c|c|c|c|}
\hline \multirow[b]{3}{*}{ Category } & \multicolumn{4}{|c|}{ Palatability rating $^{1}$} & \multicolumn{4}{|c|}{ Calorific value rating ${ }^{2}$} \\
\hline & \multicolumn{2}{|c|}{ Healthy-weight } & \multicolumn{2}{|c|}{ Overweight } & \multicolumn{2}{|c|}{ Healthy-weight } & \multicolumn{2}{|c|}{ Overweight } \\
\hline & $M$ & $S D$ & $M$ & $S D$ & $M$ & $S D$ & $M$ & $S D$ \\
\hline $\begin{array}{l}\text { High-calorie, ranked } \\
\text { most palatable }\end{array}$ & 83.7 & 11.0 & 83.0 & 15.7 & 77.5 & 15.5 & 81.3 & 13.3 \\
\hline $\begin{array}{l}\text { High-calorie, ranked } \\
\text { least palatable }\end{array}$ & 39.4 & 23.3 & 39.0 & 21.4 & 65.3 & 10.7 & 64.6 & 20.5 \\
\hline $\begin{array}{l}\text { Low-calorie, ranked } \\
\text { most palatable }\end{array}$ & 86.6 & 12.3 & 88.1 & 13.7 & 19.4 & 20.4 & 23.5 & 13.5 \\
\hline $\begin{array}{l}\text { Low-calorie, ranked } \\
\text { least palatable }\end{array}$ & 35.9 & 21.8 & 35.4 & 21.7 & 23.8 & 17.0 & 23.3 & 13.4 \\
\hline
\end{tabular}

${ }^{1}$ Rating on $100 \mathrm{~mm}$ VAS scales, ranging from 0 'not delicious at all' to 100 'very delicious'.

${ }^{2}$ Rating on 100 mm VAS scales, ranging from 0 'very low in calories' to 100 'very high in calories'.

$F(1,27)=.009, p=.924$ or calorific value, $F(1,27)=2.203, p=.149$. This means that the palatable and unpalatable stimuli differed in palatability as intended, independent of the calorific value for all participants. There was no main effect of calorific value, $F(1,27)=.006, p=.940$.

The calorific value ratings showed that the high-calorie stimuli were judged as being higher in calorific value than the low-calorie stimuli, $F(1,27)=172.74, p<.001$. A significant main effect of palatability was also found, $F(1,27)=6.96, p=.014$, and this effect was qualified by a calorific value $\times$ ranking palatability interaction, $F(1,27)=9.07$, $p=.006$. Further $t$ tests showed that there was a difference in calorific value ratings between most and least palatable high-calorie food stimuli, $t(28)=3.41, p=.002$, but no difference between the most and least palatable low-calorie food stimuli, $t(28)=.79, p=.437$. None of the effects were moderated by the factor group, all $F<.939$, all $p>.341$.

\section{fMRI Results}

To test the hypothesis that group differences in brain activity in response to viewing highcalorie palatable food stimuli would be more pronounced in the unbiased viewing condition than in the taste imagination condition, the group $\times$ attention type interaction was tested for brain responses to high-calorie palatable food stimuli. In addition to regions outside the reward system (see Table 3), fourteen fROIs belonging to or associated with the reward system (see Table 4) were found. These fROIs were located in the amygdala, posterior fusiform gyrus and dorsolateral prefrontal cortex (dlPFC) of both hemispheres, the right anterior insula and orbitofrontal cortex (OFC), the bilateral ventral tegmental area (VTA) and substantia nigra (SN), right parahippocampal gyrus, and bilateral medial prefrontal cortex (mPFC) containing anterior midcingulate and paracingulate cortex. 
Table 3. Clusters in non-reward regions with a significant interaction of group (healthy-weight, overweight) and attention type (unbiased viewing, taste imagination) during viewing of highcalorie palatable foods

\begin{tabular}{|c|c|c|c|c|c|c|c|}
\hline \multirow{2}{*}{ Anatomical label } & \multirow{2}{*}{$\begin{array}{l}\text { estimated } \\
\text { BA }\end{array}$} & \multirow{2}{*}{$\begin{array}{c}\text { size } \\
\left(\mathrm{mm}^{3}\right)\end{array}$} & \multicolumn{5}{|c|}{ Peak voxel values ${ }^{1}$} \\
\hline & & & $\mathrm{x}$ & $\mathrm{y}$ & $\mathrm{z}$ & $F$ & $p$ \\
\hline cingulate gyrus, medFG, MFG, SFG R & $6,8,24$ & 1175 & 21 & 11 & 60 & 23.66 & 0.00004 \\
\hline $\begin{array}{l}\text { thalamus (medial dorsal nuclei) } \\
\text { (bilateral) }\end{array}$ & & 429 & 3 & -11 & 4 & 23.11 & 0.00005 \\
\hline cuneus, middle occipital gyrus L & $17,18,19$ & 375 & -29 & -91 & 6 & 21.92 & 0.00007 \\
\hline precentral gyrus L & 4,6 & 86 & -61 & -1 & 16 & 17.80 & 0.00023 \\
\hline SMG, IPL L & 40 & 194 & -43 & -49 & 34 & 17.53 & 0.00025 \\
\hline postcentral gyrus, precentral gyrus L & 3,4 & 163 & -17 & -27 & 68 & 16.82 & 0.00032 \\
\hline STG R & 22 & 96 & 61 & -53 & 14 & 15.99 & 0.00042 \\
\hline STG R & 41 & 73 & 37 & -35 & 8 & 15.70 & 0.00046 \\
\hline angular gyrus, MTG, STG, SMG R & 39,40 & 441 & 53 & -61 & 28 & 15.58 & 0.00049 \\
\hline medFG, SFG L & 6 & 143 & -17 & 11 & 54 & 14.65 & 0.00067 \\
\hline cuneus, lingual gyrus R & 17 & 251 & 17 & -93 & 4 & 14.33 & 0.00074 \\
\hline IPL, STG, SMG L & $13,22,40$ & 318 & -53 & -45 & 22 & 14.31 & 0.00075 \\
\hline entorhinal cortex L & 28,34 & 148 & -11 & -7 & -24 & 14.29 & 0.00076 \\
\hline IPL, SMG R & 40 & 77 & 51 & -45 & 38 & 14.24 & 0.00077 \\
\hline precentral gyrus L & 6 & 83 & -41 & -1 & 30 & 14.03 & 0.00083 \\
\hline IFG R & 45 & 72 & 49 & 27 & 4 & 14.02 & 0.00083 \\
\hline SMG R & 31,40 & 294 & 29 & -47 & 32 & 14.01 & 0.00083 \\
\hline inferior temporal gyrus, MTG L & 20,21 & 92 & -51 & -11 & -18 & 14.01 & 0.00083 \\
\hline postcentral gyrus, precuneus L & $3,5,7$ & 165 & -15 & -41 & 64 & 13.88 & 0.00087 \\
\hline inferior and middle occipital gyrus $\mathrm{R}$ & 18,19 & 484 & 41 & -81 & -2 & 13.24 & 0.00110 \\
\hline thalamus and lateral geniculum body L & & 72 & -25 & -23 & 0 & 13.23 & 0.00110 \\
\hline middle occipital gyrus L & 19 & 119 & -33 & -73 & 4 & 12.46 & 0.00146 \\
\hline $\begin{array}{l}\text { fusiform gyrus, inferior occipital } \\
\text { gyrus, lingual gyrus L }\end{array}$ & 17,18 & 119 & -19 & -95 & -10 & 11.88 & 0.00181 \\
\hline inferior occipital gyrus L & 18 & 129 & -35 & -85 & -6 & 11.06 & 0.00247 \\
\hline
\end{tabular}

Abbreviations. $\mathrm{BA}=$ Brodmann Area; $\mathrm{IFG}=$ inferior frontal gyrus; $\mathrm{IPL}=$ inferior parietal lobule; $\mathrm{L}=$ left hemisphere; medFG = medial frontal gyrus; $\mathrm{MFG}=$ middle frontal gyrus; $\mathrm{MTG}=$ middle temporal gyrus; $\mathrm{R}=$ right hemisphere; SFG = superior frontal gyrus; SMG = supramarginal gyrus; $S T G$ = superior temporal gyrus.

${ }^{1}$ Voxel coordinates are reported in Talairach space. 
Table 4. Clusters in reward regions and associated regions with a significant interaction of group (healthy-weight, overweight) and attention type (unbiased viewing, taste imagination) during viewing of high-calorie palatable foods

\begin{tabular}{|c|c|c|c|c|c|c|c|c|}
\hline \multirow{2}{*}{ 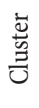 } & \multirow{2}{*}{$\begin{array}{l}\text { Anatomical label of a priori regions } \\
\text { (fROIs) }\end{array}$} & \multirow{2}{*}{$\begin{array}{l}\text { esti- } \\
\text { mated } \\
\text { BA }\end{array}$} & \multirow{2}{*}{$\begin{array}{l}\text { size } \\
\left(\mathrm{mm}^{3}\right)\end{array}$} & \multicolumn{5}{|c|}{ Peak voxel values ${ }^{1}$} \\
\hline & & & & $\mathrm{x}$ & $\mathrm{y}$ & $\mathrm{z}$ & $F$ & $p$ \\
\hline A & $\begin{array}{l}\text { amygdala (basolateral, basomedial } \\
\text { and lateral nucleus) L }\end{array}$ & & 458 & -19 & -3 & -14 & 23.39 & 0.00004 \\
\hline B & $\begin{array}{l}\text { cingulate gyrus, medial frontal gyrus } \\
\text { (dmPFC, ACC) (bilateral) }\end{array}$ & $\begin{array}{r}9,32 \\
6,8\end{array}$ & 792 & 3 & 33 & 32 & 17.86 & 0.00023 \\
\hline $\mathrm{C}$ & posterior fusiform gyrus $\mathrm{R}$ & 19 & 80 & 47 & -67 & -14 & 17.53 & 0.00025 \\
\hline $\mathrm{D}$ & IFG (mid-caudal OFC) $\mathrm{R}$ & 47 & 190 & 19 & 31 & -6 & 16.27 & 0.00038 \\
\hline E & VTA and SN (bilateral) & & 195 & 1 & -17 & -12 & 15.31 & 0.00053 \\
\hline F & MFG (dlPFC) R & 9 & 94 & 41 & 17 & 30 & 13.95 & 0.00085 \\
\hline G & medFG (anteromedial OFC) R & 10 & 107 & 5 & 55 & -6 & 13.72 & 0.00092 \\
\hline $\mathrm{H}$ & rostral ACC L & 32 & 87 & -13 & 31 & 20 & 13.62 & 0.00096 \\
\hline I & $\begin{array}{l}\text { amygdala (basolateral and lateral } \\
\text { nucleus) R }\end{array}$ & & 137 & 23 & -1 & -16 & 12.81 & 0.00128 \\
\hline $\mathrm{J}$ & parahippocampal gyrus $\mathrm{R}$ & & 121 & 35 & -7 & -24 & 12.66 & 0.00141 \\
\hline K & middle frontal gyrus (dlPFC) R & 9 & 65 & 53 & 15 & 34 & 12.54 & 0.00142 \\
\hline $\mathrm{L}$ & $\begin{array}{l}\text { anterior insula (anterior peri-insular } \\
\text { sulcus and accessory gyrus) and IFG } \\
\text { (vlPFC) R }\end{array}$ & 13,47 & 187 & 33 & 23 & 0 & 12.27 & 0.00157 \\
\hline M & posterior fusiform gyrus L & 19 & 96 & -37 & -79 & -14 & 12.17 & 0.00162 \\
\hline $\mathrm{N}$ & MFG (dlPFC) L & 9,46 & 169 & -39 & 25 & 26 & 11.60 & 0.00201 \\
\hline
\end{tabular}

Abbreviations $. \mathrm{BA}=$ Brodmann Area $\mathrm{ACC}=$ anterior cingulate cortex; $\mathrm{dlPFC}=$ dorsolateral prefrontal cortex; $\mathrm{dmPFC}=$ dorsomedial prefrontal cortex; fROI = functional region of interest; IFG = inferior frontal gyrus; $\mathrm{L}=$ left hemisphere; medFG = medial frontal gyrus; $\mathrm{MFG}=$ middle frontal gyrus; $\mathrm{OFC}=$ orbitofrontal cortex; $\mathrm{R}=$ right hemisphere; $\mathrm{SN}=$ substantia nigra; $\mathrm{vlPFC}=$ ventrolateral prefrontal cortex; VTA = ventral tegmental area.

When looking at the beta values of these clusters in the respective attention conditions and groups, the response pattern was similar in all clusters, as shown in Figure 2. In the unbiased viewing condition, the healthy-weight participants showed a greater response to high-calorie palatable food stimuli than did the overweight ones. In the taste imagination condition the pattern of results was exactly the opposite: the overweight participants showed a greater response to high-calorie palatable food stimuli than did the healthy-weight ones. Therefore the results in the taste imagination condition were in line with our hypothesis, whereas in the unbiased viewing condition they were the opposite from our hypothesis. 
To check whether the fROIs resulting from the interaction of group $\times$ attention type were specific to high-calorie palatable food stimuli, we determined the overlap with fROIs in the reward system with the same interaction in response to low-calorie palatable and to high-calorie unpalatable food stimuli. This revealed one reward-relevant region, the left amygdala, which showed an interaction of group $\times$ attention type in response to all three types of food stimuli. The response in this region to high-calorie unpalatable and low-calorie palatable food stimuli was similar to the reaction to high-calorie palatable food stimuli. In summary, in all fROIs except for the left amygdala, the group $\times$ attention type interaction was specific to high-calorie palatable food stimuli.

\section{Discussion}

The present study investigated the role of BMI and attention in the reward processing of palatable high-calorie food stimuli. In line with the existing literature we hypothesised that there would be increased activity in brain reward circuitry in response to highcalorie palatable food stimuli in overweight participants compared to healthy-weight ones. Furthermore, we expected these differences to be more pronounced in the unbiased viewing condition than in the taste imagination condition. The results were partly in line with our hypothesis: During taste imagination the activity in the reward circuit of overweight participants in response to high-calorie food stimuli was greater than in healthy-weight participants. During unbiased viewing, the results were the opposite of our hypothesis: In this condition, the overweight participants showed a weaker response to high-calorie food stimuli than did the healthy-weight ones. These results were specific to the high-calorie palatable food stimuli, though in the amygdala a similar pattern of results was obtained for both the high-calorie unpalatable and low-calorie palatable food stimuli. This implies that the affective processing in the amygdala is not dependent on valence when it comes to seeing food cues, a finding also observed when tasting pleasant and unpleasant liquids (Small, Gregory, et al., 2003).

That the overweight participants showed less reward activity than the healthyweight in the unbiased viewing condition was unexpected. One possible explanation is that the overweight participants attended to health aspects during unbiased viewing whereas the healthy-weight participants focused on the good taste of the high-calorie palatable foods, leading to this unexpected pattern of results. Note that overweight participants scored higher on a measure of dietary restraint in this study as well as in previous studies (Herman \& Polivy, 1980; Van Strien et al., 2007), which possibly contributed to them paying attention to health aspects during unbiased viewing and attentionally avoiding the (palatability aspects of the) presented food stimuli. 
Reward activity in satiated overweight women is decreased during unbiased viewing but increased when imagining taste: an event-related fMRI study

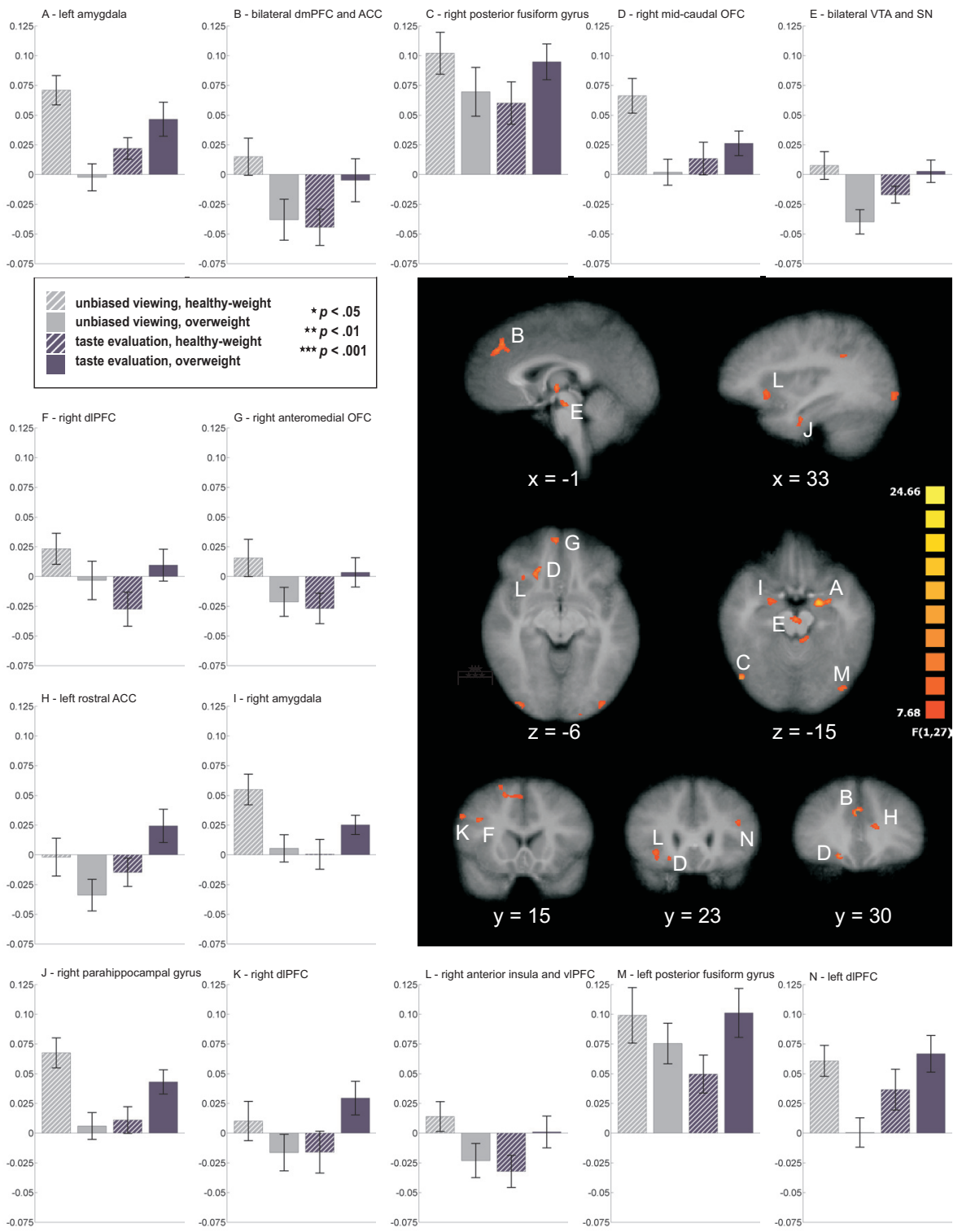

Figure 2. Locations and beta values of clusters in regions associated with the reward system with a significant group $\times$ attention type interaction in response to high-calorie palatable food stimuli, overlayed on a brain averaged from all participants, shown in radiological convention. Bar plots indicate z-scores of beta values \pm S.E.M. ACC $=$ anterior cingulate cortex; $\mathrm{dlPFC}=$ dorsolateral prefrontal cortex; $\mathrm{dmPFC}=$ dorsomedial prefrontal cortex; $\mathrm{OFC}=$ orbitofrontal cortex; $\mathrm{SN}=$ substantia nigra; $\mathrm{vlPFC}=$ ventrolateral prefrontal cortex; VTA $=$ ventral tegmental area. 
Such an attentional avoidance of palatability during unbiased viewing is corroborated by a previous study reporting attentional avoidance of high-calorie food in unsuccessful dieters (Veenstra, De Jong, Koster, \& Roefs, 2010). In the taste imagination condition, attentional avoidance was prevented by the instructions to focus on the taste of the stimuli. If, during unbiased viewing, overweight participants in this study were indeed attentionally avoiding, they likely did so in an automatic way, because the fROIs with a control exerting function (e.g. three fROIs found in the dlPFC) did not exhibit a stronger response in the overweight participants during unbiased viewing than during taste imagination. If there had been a cognitive effort of avoidance during unbiased viewing, it is likely that these regions in the dlPFC would have shown more activation during unbiased viewing than during taste imagination. An automatic avoidance by the overweight participants in the unbiased viewing condition is in line with recent findings of our laboratory concerning reduced maintenance of attention on food pictures by overweight compared to healthy-weight participants (Werthmann et al., 2011).

Two alternative explanations for these results arise from the non-counterbalanced design of the study: unbiased viewing always preceded taste imagination. Previous studies have shown that there is a slower habituation to a taste stimulus in obese participants as compared to lean ones (Bond et al., 2009), as well as a greater effect of sensitization on responding for food in overweight versus non-overweight children (Epstein et al., 2008). Therefore, it is possible that the overweight participants were sensitised by the unbiased viewing condition and therefore showed an elevated response during taste imagination, whereas the healthy-weight were habituated after unbiased viewing and therefore showed a decreased response during taste imagination. In addition, due to the elapsed time during runs 1 and 2 (approximately 35 minutes of unbiased viewing), hunger sensations may have differed between groups during the taste imagination condition, although they were similar at the start of the scanning session. Increased hunger in the overweight group could thus have lead to the elevated response during taste imagination in this group.

The event-related design likely contributed to the results during unbiased viewing, in that there was less reward activation in overweight participants compared to healthyweight ones. These findings differ from other studies in which a blocked design was used (McCaffery et al., 2009; Rothemund et al., 2007; Stoeckel et al., 2008; Wallner-Liebmann et al., 2010) and the opposite was found, i.e. greater reward activation in those who were overweight. Assuming that craving is stronger when consecutively viewing multiple high-calorie palatable food pictures (blocked design) versus seeing them intermittently mixed with pictures of another category (event-related design), a blocked design may seduce participants to indulge in craving more so than an event-related design, even if participants are not explicitly instructed to do so. One may therefore argue that an eventrelated design gives a more accurate measurement of food reward-related brain activity because the reward-value of individual food stimuli is assessed. In a blocked design one can only measure the brain activity in response to a group of stimuli. 
During taste imagination in a state of satiety, brain reward activation in response to high-calorie palatable food stimuli was higher in the overweight participants than in the healthy-weight ones, which was in line with our hypothesis and with previous studies using a blocked design (Rothemund et al., 2007; Stoeckel et al., 2008). This higher activation is thought to reflect a stronger motivation to approach or consume high-calorie palatable foods, although participants were satiated.

The strengths of this study are that we showed how different attention types can modulate brain reward responses in groups with a different BMI with the use of an eventrelated design. However, several limitations are also present. First, to avoid carry-over effects of taste imagination on unbiased viewing, conditions were not counterbalanced. This design choice has the disadvantage that we cannot exclude order effects, as described above. Second, no data were collected about what participants were thinking during unbiased viewing nor did we check that they did indeed imagine the taste of the food stimuli. Third, there were only four obese (BMI > 30) participants in the overweight group; the remaining ten participants in this group had a BMI between 27 and 30. With a group of obese participants, results may have differed.

The question remains how the present results contribute to the issue of pathological overeating. To be able to further generalise these results to a larger population, a replication of this study in obese as well as in males is required because men and women have been found to differ in brain activation to taste, both during hunger and satiety (Haase, Green, \& Murphy, 2011; Uher, Treasure, Heining, Brammer, \& Campbell, 2006). With the current results we may speculate that the large difference in reward region activation in overweight women found in this study, depending on whether food cues are viewed without instructions or with the explicit instruction to imagine their taste, represents the likely ambivalence between desire for (in the taste imagination condition) and avoidance of (in the unbiased viewing condition) high-calorie palatable food stimuli. This ambivalence may be the cause of the addictive eating pattern of overweight people (A. J. Hill, 2007; Rogers \& Smit, 2000), which may be a relevant clue for therapy research as well.

\section{ACKNowledgements}

We would like to thank Ing. Charlie Bonnemayer of Maastricht University for his help in programming the stimulus protocol in E-Prime, Dr. Armin Heinecke of Brain Innovation B.V. for his help with the data analysis, and Dr. Samantha Brooks for proofreading of the manuscript. This study was financed by the Netherlands Research Organization (NWO) grant number 400.06.148, awarded to Prof. Dr. Anita Jansen. 



\title{
Chapter 3
}

\section{REWARD OR INHIBITION}

\author{
WHEN SEEING PALATABLE FOOD?
}

IT ALL DEPENDS ON YOUR WEIGHT

AND YOUR FOCUS OF ATTENTION

Frankort, A., Roefs, A., Siep, N., Roebroeck, A., Havermans, R., \& Jansen, A. (n.d.). Reward or inhibition when seeing palatable food? It all depends on your weight and your focus of attention. Manuscript in preparation. 


\begin{abstract}
This study investigated if, on a neural and on a subjective level, overweight participants are more responsive to palatability than healthyweight participants. To this end, subjective and neural responses to palatable and neutral food cues were measured in overweight and healthy-weight participants in an event-related design in two conditions: unbiased viewing without prior instructions and taste imagination. It was expected that group differences would not be found in subjective reports, but would exist on a neural level: overweight participants were expected to show more reward-related brain activation, and less inhibitory activation, than healthy-weight participants when seeing palatable versus neutral food cues. When viewing the stimuli naturally without instructions, results contradicted our hypothesis: in 7 fROIs associated with reward, the response to palatable versus neutral food was smaller in overweight compared with healthy-weight participants. In 3 putative inhibitory fROIs, the same response was larger in the overweight participants. In the taste imagination condition, results were in line with our hypothesis. Here, 7 reward-related fROIs were more active in response to palatable versus neutral food stimuli in overweight compared with healthy-weight participants. These results show that when overweight participants view food stimuli without being biased by instructions, their brain activity is very different than when they focus on the taste of the presented food stimulus: During unbiased viewing inhibitory processes are dominant, whereas reward-related processes are dominant during taste imagination. This study adds to the limited evidence-base that attention focus is highly relevant in the study of food reward and inhibition.
\end{abstract}




\section{INTRODUCTION}

In a previous paper (Frankort et al., 2012), we reported analyses of brain responses to high-calorie palatable food cues in healthy-weight and overweight participants during two attention conditions. In a first attention condition, unbiased viewing, participants were not biased or instructed on how they should perceive the presented food stimuli. In a second condition, taste imagination, participants were asked to imagine the taste of the presented food stimuli. A whole-brain analysis of the interaction between group (healthy weight vs. overweight) and attention condition on the responses to high-calorie palatable food cues resulted in several functional regions of interest (fROIs) involved in reward. Further inspection of these fROIs revealed that the activation in the overweight participants was higher than in the healthy-weight participants during taste imagination, whereas during unbiased viewing the opposite result was found: In this condition, the overweight participants had a lower activation in fROIs associated with reward than the healthy-weight participants. It was concluded that the overweight participants showed an ambivalent activation in response to high-calorie palatable food stimuli: a heightened desire during taste imagination and avoidance during unbiased viewing.

In Frankort et al. (2012) we only looked at the response to high-calorie palatable food stimuli. So, using whole-brain analyses, only fROIs that responded to this type of food stimuli were selected, because high-calorie palatable food is considered to be linked to the consumption of energy-dense palatable foods (Drewnowski, 2007). However, in the eventrelated design of that study also low-calorie and less palatable food stimuli were presented. In Frankort et al. (2012) it was only checked qualitatively if the observed activation in the found fROIs was unique to high-calorie palatable foods, or whether the same clusters would have emerged if the analysis was done with low-calorie palatable or high-calorie unpalatable food stimuli. This was largely not the case, so the found fROIs were unique to high-calorie palatable food stimuli. The analyses with the other stimulus types were not regarded further.

So, in Frankort et al. (2012) an analysis of possible group (overweight vs. healthyweight) differences in response to variations in palatability was not conducted, although this would be relevant and interesting. When it comes to food consumption, there is quite some evidence that obese participants (as compared to healthy-weight controls) eat a larger amount of good tasting food, and an equal or smaller amount of unpalatable food (Pliner et al., 1990), whereas this interactive effect is not found in the subjective reports of liking of food items (Dimitropoulos et al., 2012; Frankort et al., 2012; Nummenmaa et al., 2012; Rothemund et al., 2007; Scharmüller et al., 2012). By directly assessing food-cue elicited brain processing, more light might be shed on this inconsistency in behaviour versus self-reported liking.

Therefore we chose to re-analyse the data of Frankort et al. (2012) with the following changes with regard to the original analysis. First, high-calorie and low-calorie food items were pooled, because our interest was on variations of palatability, not on calorific 
value. Second, additionally to responses to very palatable food stimuli, food stimuli regarded as neutral were included. Stimuli regarded as unpalatable were not taken into account to study different parts of the data than in Frankort et al. (2012) and to avoid aversion effects. Note that after the scanning session, the food cues had been assigned to palatable, neutral or unpalatable subsets according to individual taste preferences. Subsets therefore were different for every participant. Third, fROIs were selected in wholebrain analyses using the interaction between group (overweight vs. healthy-weight) and palatability (very palatable vs. neutral), separately for the two conditions of attention.

We expected that the subjective palatability ratings of very palatable and neutral stimuli, which were collected after the scanning session, would not differ between groups, whereas the neural response to very palatable versus neutral food pictures would be larger in overweight than in healthy-weight participants. This effect of increased brain activity in response to palatable versus neutral foods in overweight people was expected to occur in brain regions involved in food reward, like the striatum, hippocampus, amygdala, insula, substantia nigra and ventral tegmental area, as well as anterior cingulate, prefrontal, posterior fusiform and orbitofrontal cortices (for reviews see: García-García, Narberhaus, et al., 2013; Kringelbach, 2009; Rolls, 2011; Van der Laan, De Ridder, Viergever, \& Smeets, 2011). Moreover, we expected the opposite pattern of results in brain regions associated with inhibitory control. These brain areas include subdivisions of the prefrontal cortex (PFC) like medial, ventrolateral and dorsolateral PFC, as well as posterior parietal cortex (Kishinevsky et al., 2012; Stoeckel et al., 2012; Yokum et al., 2011a). In these inhibitory regions, less activation was expected in overweight compared with healthy-weight participants. The attention manipulation (unbiased viewing vs. taste imagination) was hypothesised to play a moderating role: the expected increased reward processing in response to palatable versus neutral foods in overweight compared with healthy-weight participants was thought to occur especially during the condition of taste imagination.

\section{METHOD}

Part of these data have already been published in a previous paper (Frankort et al., 2012), which analysed the interaction between group (overweight vs. healthy-weight) and attention type (unbiased viewing vs. focus on palatability) for high-calorie palatable food cues only. To see whether the found fROIs were specific to high-calorie palatable food cues or whether they overlapped with clusters that resulted from the same interaction for other types of food stimuli, qualitative analyses were conducted for palatable low-calorie and unpalatable high-calorie foods as well. The current study focuses on group differences between palatable versus neutral food cues, with pooled levels of calorific density, separately for each of the attention conditions. Responses to unpalatable food cues were not included in the current study. 


\section{Participants}

15 overweight (body mass index $(\mathrm{BMI})>25)$ and 15 healthy-weight $(18 \leq \mathrm{BMI} \leq 25)$ Dutch women participated in this study. One overweight participant was excluded from analyses due to scanner problems. Participants were recruited among students and employees from Maastricht University and the general population. Groups were matched on education level and showed no group differences regarding age, hunger or affect. Groups differed significantly regarding BMI and restraint. For relevant statistics, see Table 1 . The participants had no self-reported history of mental disorders or a family history of eating disorders, were right-handed, not vegetarian, not currently on a weight-loss diet and used a one-phase oral contraceptive to ensure a relatively stable hormonal balance (Guillebaud, 1987). Remuneration for participation was $€ 30$ or course credits.

\section{Assessment}

Only variables that are relevant to the present paper are reported below. For a complete listing of assessed variables please see Frankort et al. (2012).

BMI. Body mass index $\left[\mathrm{kg} / \mathrm{m}^{2}\right]$ was determined by measuring participant's weight and height. Hunger. Four VAS items of a hunger questionnaire (Friedman et al., 1999) were used to measure hunger. The total score is the average of these four items and can range from 0 'not hungry at all' to 100 'very hungry'.

Table 1. Participant characteristics

\begin{tabular}{|c|c|c|c|c|c|c|}
\hline & \multicolumn{2}{|c|}{ Healthy-weight } & \multicolumn{2}{|c|}{ Overweight } & \multirow[b]{2}{*}{$t(27)$} & \multirow[b]{2}{*}{$p$} \\
\hline & $M$ & $S D$ & $M$ & $S D$ & & \\
\hline Age (yrs) & 23.1 & 5.4 & 23.9 & 7.6 & 0.35 & 0.73 \\
\hline BMI & 21.1 & 1.1 & 29.8 & 3.3 & 9.43 & $<.001$ \\
\hline Hunger $^{1}$ & 20.8 & 8.7 & 17.9 & 9.9 & 0.84 & 0.41 \\
\hline Restraint $^{2}$ & 8.8 & 3.1 & 13.9 & 3.2 & 4.35 & $<.001$ \\
\hline
\end{tabular}

Note. Reprinted with permission from Frankort et al. (C) 2012 International Journal of Obesity.

Abbreviations. BMI = Body Mass Index.

${ }^{1}$ Average score of four $100 \mathrm{~mm}$ VAS (hunger, fullness, desire to eat, and amount of food that could currently be eaten), ranging from 0 to 100, with a higher score representing a higher level of hunger. One of the subscales (fullness) was likely filled out inversely by seven participants so these scores were corrected; however, correction did not affect group similarity (before correction, mean hunger ratings were $23.2 \pm 9.8$ (healthy-weight) and $22.8 \pm 12.6$ (overweight), $t(27)=0.10, p=0.92$ ).

${ }^{2}$ Scored on the Restraint Scale (Herman \& Polivy, 1980), with a minimum total score of 0 (no restraint) and a maximum of 35 (high restraint). 
Restraint. The Restraint Scale (Herman \& Polivy, 1980) measures the chronic intention to diet. The total score can range between 0 and 35, with higher scores reflecting more intentions to restrain food intake. This scale is sufficiently valid and reliable (Scagliusi et al., 2005; Van Strien et al., 2007; Williamson et al., 2007).

Stimuli. Stimuli were 32 pictures of 16 different food items ( 2 different pictures of each food item), purchased online (www.istockphoto.com). Half of the food items were highcalorie and half were low-calorie. By means of each participant's ranking of palatability (see next paragraph), idiosyncratic sets of most palatable, neutral, and least palatable food stimuli were created. For the current study, high-calorie and low-calorie food items of the same palatability (palatable, neutral or unpalatable) were pooled and only responses to palatable and neutral stimuli were included in the analyses.

Palatability ranking of stimuli. To obtain idiosyncratic subsets of food items, all food items were ranked on palatability. This was done separately for high-calorie and low-calorie items. Items with pictures that remained unrecognised were assigned to the neutral subset. This resulted in six subsets: most palatable, neutral and least palatable for both high-calorie and low-calorie foods, containing 3, 2 and 3 food items, respectively, with 2 different pictures per food item. For the current study, high-calorie and low-calorie subsets of each palatability level were combined and only responses to most palatable and neutral food items were taken into account.

Palatability rating of stimuli. Palatability of the stimuli was assessed on $100 \mathrm{~mm}$ VAS.

\section{Experimental Design}

The data were analysed in a mixed 2 (BMI: healthy-weight vs. overweight) $\times 2$ (palatability: palatable vs. neutral) design, so the factor calorific value was collapsed. This was done separately for the unbiased viewing condition and for the taste imagination condition.

\section{Stimulation Protocol}

The stimulation protocol was programmed in Eprime (PST, 1996). The presentation duration of the stimuli was $2000 \mathrm{~ms}$. The inter-stimulus interval (ISI) was 2, 4, 6, or $8 \mathrm{~s}$, during which a small white fixation cross was presented on a black background. Each of the 16 different food items was shown four times per run and was preceded by a different ISI before every presentation (Serences, 2004). Stimuli were shown in cycles of eight food items with four high-calorie and four low-calorie items each. At the end of each cycle, an overview of the preceding eight stimuli was shown, out of which the participant was asked to choose three (see Figure 1). In runs 1 and 2 (unbiased viewing condition), no further instructions were provided for the perception of the stimuli or for the choice task. In runs 3 and 4 (taste imagination condition), participants were instructed to pay attention to the taste of the food stimuli and to choose the three most palatable food items from the overview. Selection of 
three stimuli was done by means of two button boxes (one for each hand). The two conditions were not counterbalanced over runs, because of possible carry-over effects from the taste imagination condition to the taste unbiased viewing condition. Per functional run, eight cycles of eight food items were shown. The functional run duration varied due to the variable time needed for item selection from the overview screen, and amounted to approximately $12 \mathrm{~min}$. One fMRI session consisted of four functional runs and one anatomical run.

\section{Procedure}

The scanning session was scheduled in the early afternoon. The participant was requested to consume a regular lunch one to 1.5 hours before entering the scanner. Before the start of the session, subjective hunger ratings, affect, weight and height were measured.

After entering the scanner, the use of the two button boxes and the selection of stimuli were practised with an exercise task containing neutral non-food stimuli. The participant then underwent two functional runs, an anatomical run, and then two functional runs again. After the fMRI session, recognition of the stimuli of the scanner task was tested. At least 14 of 16 food items were recognised by all participants. Subsequently, palatability ranking and rating were done, after which restraint was measured and an exit questionnaire was filled out. Debriefing was done by e-mail at the end of the study. The study was approved by the Ethical Committee of the Faculty of Psychology and Neuroscience of Maastricht University.

\section{fMRI Data Acquisition}

A 3T Siemens Magnetom Allegra Head-only scanner was used for image acquisition, with optimised parameters for susceptibility and distortion artefact reduction in the OFC (Deichmann et al., 2003; Weiskopf et al., 2007): TE $=25 \mathrm{~ms}$, voxel size $=2 \times 2 \times 2.5 \mathrm{~mm}^{3}$, $\mathrm{TR}=2000 \mathrm{~ms}$, flip angle $=90^{\circ}, \mathrm{FoV}=256 \times 192 \mathrm{~mm}^{2}$, imaging bandwidth $=2790 \mathrm{~Hz} /$ pixel, echo spacing $=0.42 \mathrm{~ms}$, resulting in $33 \mathrm{~T} 2^{\star}$-weighted axial slices with a tilt angle of $-30^{\circ}$ and a distance factor of $40 \%$. Four functional runs per participant were acquired, with a number of volumes per run between 338 and 369. The anatomical scan was acquired using an optimised magnetization-prepared rapid gradient-echo sequence (Deichmann et al., 2000; Mugler \& Brookeman, 1990) with the following settings: $\mathrm{TR}=2250 \mathrm{~ms}, \mathrm{TE}=2.6$ ms, flip angle $=9^{\circ}$, voxel size $=1 \times 1 \times 1 \mathrm{~mm}^{3}$.

\section{Data Preprocessing}

All fMRI data were analysed with BrainVoyager QX (BrainInnovation, 2001). The first two volumes of each functional run were excluded due to T1 saturation effects. Preprocessing steps consisted of slice scan time correction with cubic spline interpolation, three-dimensional motion correction using trilinear (for alignment) and sinc (for final resampling) in- 


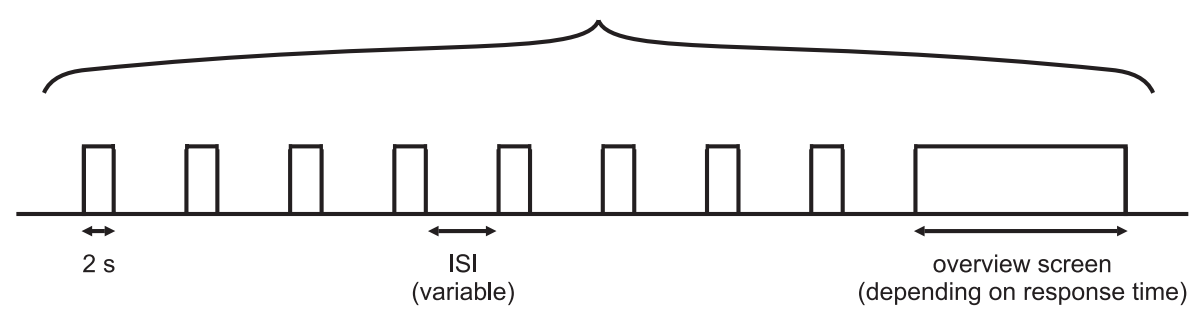

Figure 1. Graphical outline of one cycle of the fast event-related stimulation protocol, consisting of eight stimuli (half of which were low-calorie and half were high-calorie) and an overview screen. This cycle was repeated eight times within one run. Stimuli were presented during $2 \mathrm{~s}$ each. The variable inter-stimulus interval (ISI) lasted 2, 4, 6, or $8 \mathrm{~s}$, during which a small fixation cross was shown on a black background. In the overview screen, participants had to choose three of the preceding eight stimuli. Reprinted with permission from Frankort et al. ๑ 2012 International Journal of Obesity.

terpolation, high-pass temporal filtering for removal of low-frequency noise with less than two cycles in a single run time-course (between 0.0027 and $0.0030 \mathrm{~Hz}$ cut-off, depending on the run length). Subsequently, the functional data were co-registered with the anatomical scan. This resulted in three-dimensional space data over the course of time with a voxel resolution of $2 \mathrm{~mm} \times 2 \mathrm{~mm} \times 2 \mathrm{~mm}$. With the Talairach transformation procedures (Talairach \& Tournoux, 1988), all data were spatially normalised and finally smoothed with a $6 \mathrm{~mm}$ full-width-at-half-maximum isotropic Gaussian Kernel. Talairach-standardised anatomical data sets of all participants were averaged for group-level analyses. Based on this average, a whole-brain mask was generated, which contained 187621 voxels.

\section{Data Analysis}

To assess subjective ratings of stimuli, palatability ratings of the palatable versus neutral stimuli subsets were compared in a 2 (group: overweight vs. healthy-weight) $\times 2$ (palatability: palatable vs. neutral) ANOVA. As explained in the paragraph on palatability ranking, all stimulus subsets were composed individually according to each participant's personal taste preferences.

For brain responses, predictors were set for the most palatable and neutral stimuli subsets. Confound predictors were set for the least palatable food items, for the overview screen and for the motion correction parameters. All predictors except motion parameters were convolved with a standard hemodynamic response function (Friston et al., 1998). With this model, the remaining fixation times during each ISI can be regarded as the baseline. Brain responses to the food items were then analysed in a 2 (group: overweight vs. healthy-weight) $\times 2$ (palatability: palatable vs. neutral) random-effects ANOVA, separately for both attention conditions. The interaction of interest was specified as a linear contrast [(overweight most palatable + healthy-weight neutral) - (overweight neutral + healthyweight most palatable)] and tested whole-brain. By doing so, two whole-brain statistical 
$F$ maps were created (one for each condition), containing voxels of $2 \mathrm{~mm} \times 2 \mathrm{~mm} \times 2 \mathrm{~mm}$ with a $p$ value below 0.05 . After taking into account the conservative whole-brain randomeffects analyses and considering cluster size thresholds used in other comparable fMRI experiments (e.g., (Frankort et al., 2012; Rothemund et al., 2007; Stoeckel et al., 2008), a minimal cluster size of $64 \mathrm{~mm}^{3}$ was used to correct for multiple comparisons. An indication of the anatomical localization of the clusters was obtained with the Talairach Client (www.talairach.org) (Lancaster et al., 2000).

In a subsequent second-level analysis, paired samples $t$ tests were performed for every cluster in the respective condition with average beta values per group, comparing responses to palatable and neutral food cues. To be included as a functional ROI in these second-level analyses, two conditions had to be met: (1) The $t$ test of palatable versus neutral food cues should be positive and significant for the overweight group or the healthyweight group, being either an indication for support or contradiction of our hypothesis, and (2) the location of the cluster should be in a brain region known to be involved in food reward or inhibition. Clusters obviously situated in white matter or in which no BOLD response could be detected were excluded.

\section{RESULTS}

Average palatability ratings per group can be found in Table 2. In the ANOVA, a group $\times$ palatability interaction was found, $F(1,27)=4.41, p=.05$. Further $t$ tests showed that this interaction was caused by a group difference on the neutral stimuli. Healthy-weight participants rated these neutral stimuli more palatable than did the overweight participants, $t(27)=2.54, p=0.02$. No group difference was observed for the palatable stimuli.

In the unbiased viewing condition, there were ten fROIs, each with a group $\times$ palatability interaction and with a positive and significant $t$ test of palatable versus neutral food cues for one of the groups (Table 3 and Figure 2). Our hypothesis was that overweight participants would show a positive difference in response to palatable versus neutral food cues in regions associated with reward, which would be greater than the corresponding difference in healthy-weight ones. However, the three regions in which we observed this activation pattern, that is, regions in the left anterior frontopolar cortex, left lateral frontopolar cortex and frontal eye fields in right anterior $\mathrm{dmPFC}$, are usually considered inhibitory or attention-directing regions instead of reward regions (Boorman, Behrens, Woolrich, \& Rushworth, 2009; Coutlee \& Huettel, 2012; Greenberg, Esterman, Wilson, Serences, \& Yantis, 2010; Muggleton, Chen, Tzeng, Hung, \& Juan, 2010). The remaining seven fROIs with an interaction in the unbiased viewing condition had an activation pattern that contradicted our hypothesis: the response to palatable minus neutral food cues was significant and positive for the healthy-weight group (instead of the overweight group), or it was significant and negative for the overweight group (see Figure 2). These fROIs are 
part of or associated with the reward system (e.g., García-García, Narberhaus, et al., 2013): the pulvinar of the right thalamus, right dorsal striatum, left insula, right dorsal anterior cingulate cortex (ACC), left rostral ACC, right caudal posterior cingulate cortex (PCC) and left anterior PFC. Therefore, the results of activation during unbiased viewing contradicted our hypothesis, both for inhibitory and for reward regions.

In the taste imagination condition, there were seven fROIs (Table 3 and Figure 3) with a group $\times$ palatability interaction. In all seven fROIs, a positive and significant difference in response to palatable minus neutral food cues was found in the overweight group, which was larger than in the healthy-weight group, and was in line with our hypothesis. These fROIs were the right ventral striatum, right fusiform gyrus, left pulvinar, left caudal PCC, right dorsomedial PFC (dmPFC) and dorsolateral PFC (dlPFC) in both hemispheres. Finally, clusters with a group $\times$ palatability interaction that did not belong to any regions as hypothesised in the introduction are listed in Table 4.

Table 2. Average palatability ratings of palatable and neutral stimuli

\begin{tabular}{lccccc}
\hline \multirow{2}{*}{$\begin{array}{l}\text { Category (with high-calorie and } \\
\text { low-calorie stimuli pooled) }\end{array}$} & & \multicolumn{2}{c}{ Healthy-weight } & & \multicolumn{2}{c}{ Overweight } \\
\cline { 2 - 3 } \cline { 5 - 6 } & $M$ & $S D$ & & $M$ & $S D$ \\
\hline Ranked as most palatable & 85.6 & 9.2 & & 85.7 & 12.6 \\
Ranked as neutral & 66.8 & 8.6 & & 56.6 & 12.7 \\
\hline
\end{tabular}

${ }^{1}$ Rating on $100 \mathrm{~mm}$ VAS scales, ranging from 0 (not delicious at all) to 100 (very delicious). 
Table 3. Clusters in fROIs with a significant group (healthy-weight vs. overweight) $\times$ palatability (palatable vs. neutral) interaction. Cluster identifiers coincide with those used in Figures 2 and 3

\begin{tabular}{|c|c|c|c|c|c|c|c|c|c|}
\hline \multirow{2}{*}{\multicolumn{2}{|c|}{ Condition and cluster }} & \multirow{2}{*}{$\begin{array}{l}\text { Anatomical label of } \\
\text { regions (fROIs) }\end{array}$} & \multirow{2}{*}{$\begin{array}{l}\text { estima- } \\
\text { ted } \mathrm{BA}^{1}\end{array}$} & \multirow{2}{*}{$\begin{array}{c}\text { size } \\
\left(\mathrm{mm}^{3}\right)\end{array}$} & \multicolumn{5}{|c|}{ Peak voxel values ${ }^{2}$} \\
\hline & & & & & $\mathrm{x}$ & $\mathrm{y}$ & $\mathrm{z}$ & $t(56)$ & $p$ \\
\hline \multicolumn{10}{|c|}{ Clusters with an interaction during unbiased viewing } \\
\hline \multirow{3}{*}{$\begin{array}{c}\mathrm{PAL}>\text { neutr for } \\
\text { overweight }\end{array}$} & $\mathrm{uA}$ & lateral frontopolar L & 9,10 & 297 & -43 & 49 & 22 & 2.99 & 0.00412 \\
\hline & $\mathrm{uB}$ & anterior frontopolar $\mathrm{L}$ & 10,9 & 179 & -25 & 59 & 26 & 2.21 & 0.03153 \\
\hline & $\mathrm{uC}$ & anterior dmPFC R (FEF) & 8 & 130 & 13 & 54 & 40 & 2.70 & 0.00912 \\
\hline \multirow{7}{*}{$\begin{array}{l}\mathrm{PAL}>\text { neutr for } \\
\text { healthy-weight }\end{array}$} & $\mathrm{uD}$ & thalamus (pulvinar) $\mathrm{R}$ & - & 249 & 25 & -25 & 3 & -3.42 & 0.00115 \\
\hline & $\mathrm{uE}$ & dorsal striatum $\mathrm{R}$ & - & 198 & 26 & -9 & 21 & -2.94 & 0.00480 \\
\hline & $\mathrm{uF}$ & rostral ACC L & 32,9 & 174 & -15 & 32 & 21 & -2.43 & 0.01832 \\
\hline & $\mathrm{uG}$ & dorsal ACC R & 32 & 133 & 19 & 14 & 33 & -2.80 & 0.00703 \\
\hline & $\mathrm{uH}$ & anterior PFC L & 10 & 131 & -22 & 46 & 23 & -2.36 & 0.02196 \\
\hline & $\mathrm{uI}$ & caudal PCC R & 29 & 90 & 10 & -43 & 18 & -2.71 & 0.00882 \\
\hline & $\mathrm{uJ}$ & middle insula L & 13 & 66 & -39 & -1 & 17 & -2.43 & 0.01813 \\
\hline
\end{tabular}

Clusters with an interaction during taste imagination

\begin{tabular}{|c|c|c|c|c|c|c|c|c|c|}
\hline \multirow{7}{*}{$\begin{array}{c}\text { PAL > neutr for } \\
\text { overweight }\end{array}$} & tA & dmPFC R & 6 & 315 & 12 & 9 & 59 & 2.42 & 0.01888 \\
\hline & $\mathrm{tB}$ & dlPFC L & 6 & 196 & -27 & 9 & 47 & 2.60 & 0.01199 \\
\hline & $\mathrm{tC}$ & ventral striatum $\mathrm{R}$ & - & 191 & 17 & -10 & 1 & 2.68 & 0.00956 \\
\hline & $\mathrm{tD}$ & dlPFC R & 6 & 180 & 36 & -5 & 48 & 2.47 & 0.01645 \\
\hline & $\mathrm{tE}$ & fusiform gyrus R & 37 & 88 & 49 & -36 & -11 & 2.42 & 0.01890 \\
\hline & $\mathrm{tF}$ & thalamus (pulvinar) $\mathrm{L}$ & - & 85 & -20 & -23 & 13 & 2.55 & 0.01354 \\
\hline & $\mathrm{tG}$ & caudal PCC L & 29,30 & 82 & -12 & -50 & 13 & 2.46 & 0.01710 \\
\hline
\end{tabular}

PAL $>$ neutr for healthy-weight

Abbreviations. $\mathrm{BA}=$ Brodmann Area; $\mathrm{ACC}=$ anterior cingulate cortex; $\mathrm{dlPFC}=$ dorsolateral prefrontal cortex; $\mathrm{dmPFC}=$ dorsomedial prefrontal cortex; $\mathrm{FEF}=$ frontal eye fields; $\mathrm{fROI}=$ functional region of interest; $\mathrm{L}=$ left hemisphere; $\mathrm{PCC}=$ posterior cingulate cortex $\mathrm{PFC}=$ prefrontal cortex $\mathrm{R}=$ right hemisphere .

1Identified with the "nearest grey matter" option in the Talairach client (www.talairach.org) (Lancaster et al., 2000). ${ }^{2}$ Voxel coordinates are reported in Talairach space. 

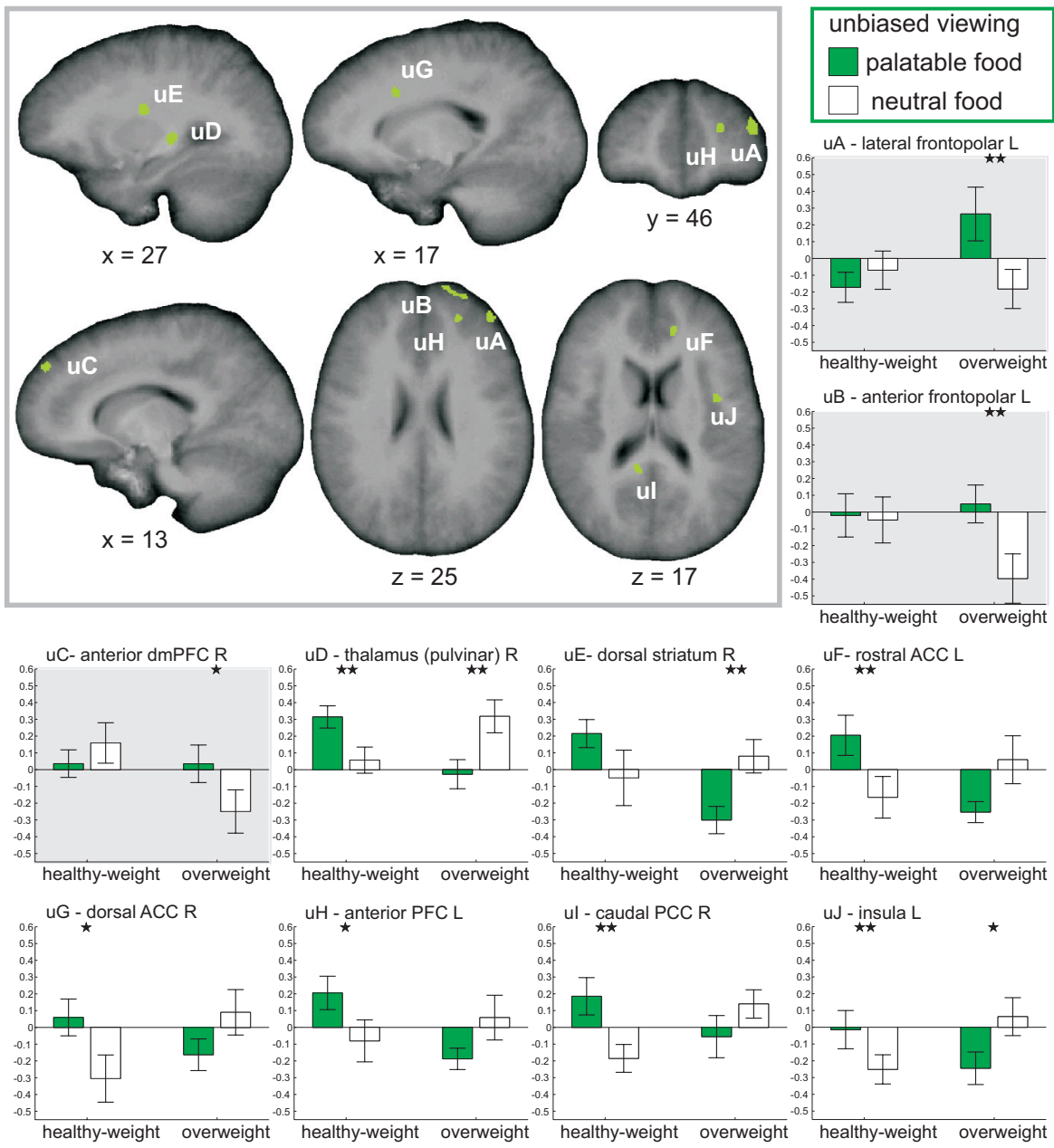

Figure 2. Upper left part: locations of fROIs with a significant group (healthy-weight vs. overweight) $\times$ palatability (palatable vs. neutral) interaction in the condition of unbiased viewing. Lower part: bar plots showing $\beta$ values \pm S.E.M., indicating group responses to palatable (green) and neutral (white) food stimuli. Bar plots of fROIs in putative inhibitory regions are shown with a grey background. Remaining regions are putative reward regions. Significance indications: ${ }^{\star} p<.05 ;{ }^{* *} p<.01 ;{ }^{* * *} p<.001$. Reported $t$ tests are uncorrected. 
Reward or inhibition when seeing palatable food? It all depends on your weight and your focus of attention
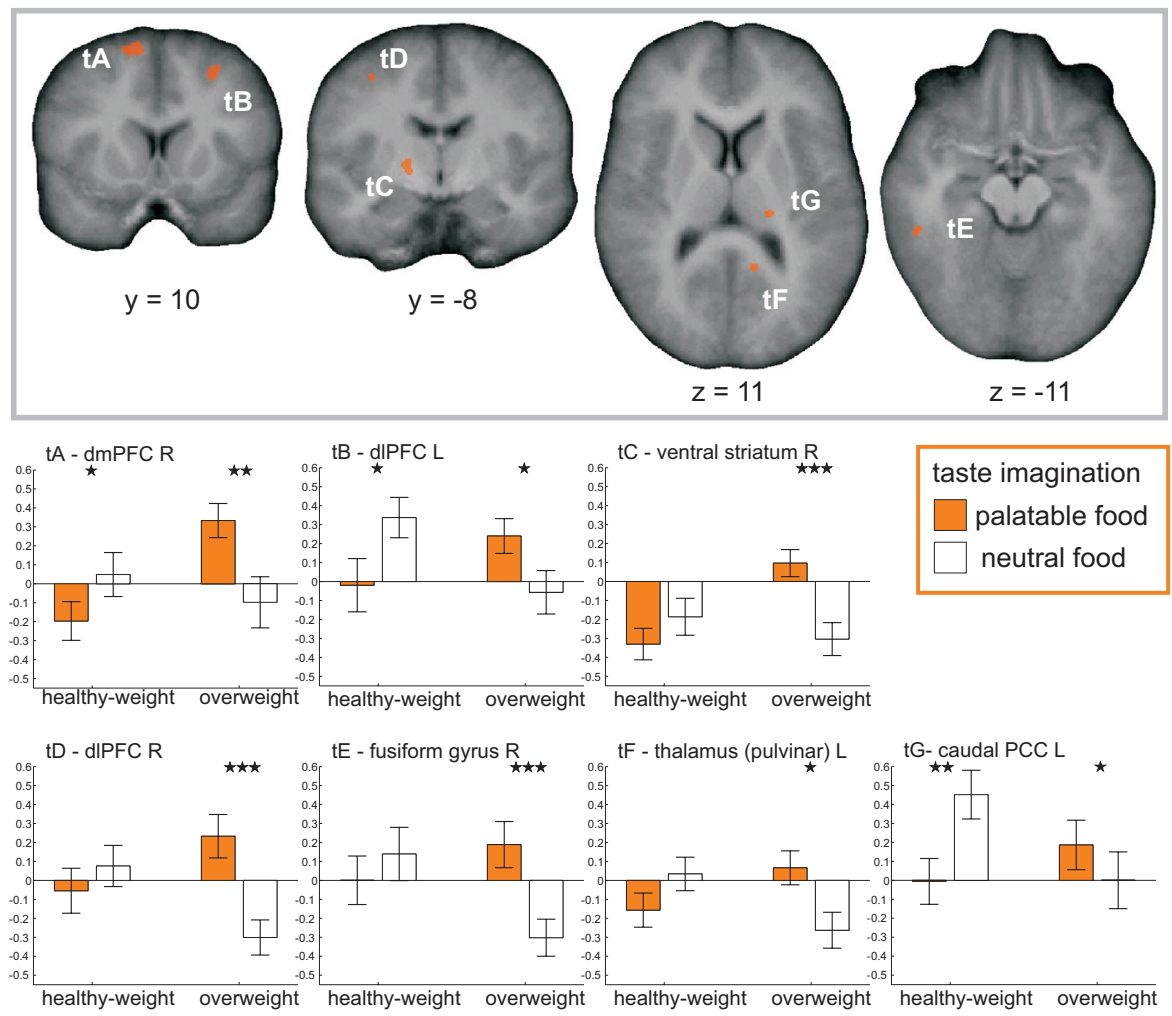

Figure 3. Upper part: locations of fROIs with a significant group (healthy-weight vs. overweight) $\times$ palatability (palatable vs. neutral) interaction in the condition of taste imagination. Lower part: bar plots showing $\beta$ values \pm S.E.M., indicating group responses to palatable (orange) and neutral (white) food stimuli. Significance indications: ${ }^{*} p<.05 ;{ }^{* *} p<.01$; ${ }^{* * *} p<.001$. Reported $t$ tests are uncorrected. 
Table 4. Remaining clusters with a significant group (healthy-weight vs. overweight) $\times$ palatability (palatable vs. neutral) interaction

\begin{tabular}{|c|c|c|c|c|c|c|c|c|}
\hline \multirow{2}{*}{ Condition and cluster } & \multirow{2}{*}{ Anatomical label } & \multirow{2}{*}{$\begin{array}{c}\text { esti- } \\
\text { mated } \\
\mathrm{BA}^{1}\end{array}$} & \multirow{2}{*}{$\begin{array}{c}\text { size } \\
\left(\mathrm{mm}^{3}\right)\end{array}$} & \multicolumn{5}{|c|}{ Peak voxel values ${ }^{2}$} \\
\hline & & & & $\mathrm{x}$ & $\mathrm{y}$ & $\mathrm{z}$ & $t(56)$ & $p$ \\
\hline \multicolumn{9}{|c|}{ Clusters with an interaction during unbiased viewing } \\
\hline $\begin{array}{l}\mathrm{PAL}>\text { neutr for } \\
\text { overweight }\end{array}$ & - & - & - & - & - & - & - & - \\
\hline \multirow{5}{*}{$\begin{array}{l}\text { PAL > neutr for } \\
\text { healthy-weight }\end{array}$} & cerebellum (medial) & - & 245 & 0 & -55 & -9 & -2.82 & 0.00668 \\
\hline & STG, tvTG L & 41 & 219 & -46 & -29 & 11 & -2.85 & 0.00615 \\
\hline & cerebellum L & - & 214 & -13 & -54 & -24 & -2.85 & 0.00600 \\
\hline & postcentral gyrus R & 40 & 86 & 61 & -20 & 17 & -2.43 & 0.01839 \\
\hline & cerebellum L & - & 66 & -5 & -41 & -8 & -2.51 & 0.01482 \\
\hline
\end{tabular}

Clusters with an interaction during taste imagination

$\begin{array}{llrrrrrrr}\begin{array}{l}\text { PAL }>\text { neutr for } \\ \text { overweight }\end{array} & \text { precentral gyrus L } & 6 & 215 & -46 & -11 & -46 & -2.84 & 0.00631 \\ & \text { cerebellum R } & - & 168 & 24 & -41 & 24 & 2.60 & 0.01190 \\ & \text { IPL R } & 40 & 105 & 52 & -44 & 52 & 2.58 & 0.01239\end{array}$

PAL $>$ neutr for

healthy-weight

postcentral gyrus $\mathrm{R}$

2

$\begin{array}{lllll}69 & 41 & -26 & 41 & 2.59\end{array}$

0.01215

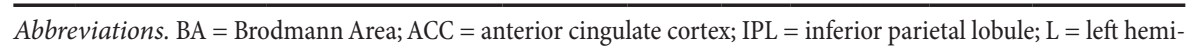
sphere; $\mathrm{PCC}=$ posterior cingulate cortex; $\mathrm{R}=$ right hemisphere; $\mathrm{STG}=$ superior temporal gyrus; tvTG = transverse temporal gyrus.

${ }^{1}$ Identified with the "nearest grey matter" option in the Talairach client (www.talairach.org) (Lancaster et al., 2000).

${ }^{2}$ Voxel coordinates are reported in Talairach space.

\section{Discussion}

This study investigated if, on a neural and on a subjective level, overweight participants are more responsive to palatability of food stimuli than are healthy-weight participants. It was hypothesised that subjective reports would not differ between groups, whereas differences were expected in directly assessed brain responses related to reward and inhibition using fMRI. More specifically, it was expected that overweight participants would show more reward-related brain activation, and less inhibitory activation, than healthy-weight participants when seeing palatable versus neutral food cues.

Regarding the subjective ratings of food stimuli, there was a marginally significant interaction between group and palatability subset, which appeared to be caused by a rat- 
ing difference of the neutral stimuli. Unexpectedly, these were rated as more palatable by healthy-weight participants than by the overweight ones. No group difference was found for the palatable stimuli.

For the taste imagination condition, fMRI-findings were in line with our hypothesis: Activation in response to palatable versus neutral food cues was greater in overweight than in healthy-weight participants in the ventral striatum, fusiform gyrus, pulvinar, caudal PCC and parts of the dorsal PFC. These brain regions have been associated with reward in previous studies. The ventral striatum and fusiform gyrus have been found to be active in response to food versus non-food stimuli in two meta-analyses (Tang, Fellows, Small, \& Dagher, 2012; Van der Laan et al., 2011). The pulvinar is involved in focused visual attention (Pessoa \& Adolphs, 2010), so more salient cues elicit a higher activation. The caudal PCC has been found to be active in response to appetizing versus bland foods (Nummenmaa et al., 2012), and in a conjunction analysis of responses to high-calorie and low-calorie foods versus control pictures (Killgore et al., 2003). The three regions in the dorsal PFC were shown to be active in response to food stimuli versus non-food stimuli or in response to taste and flavour stimuli in previous research (García-García, Jurado, et al., 2013; Kringelbach, de Araujo, \& Rolls, 2004; Martin et al., 2010).

However, the three regions in the dorsal PFC have not only been associated with food-reward in previous research, but are often considered executive control areas (Coutlee \& Huettel, 2012). So, this would mean that even in the 'focus on taste' condition, there would be evidence for an increased inhibitory response to palatable versus neutral food stimuli in overweight as compared to healthy-weight participants. However, this does not fit the increase in activation in response to palatable versus neutral food stimuli in the other fROIs, which are surely associated with food reward, as activity in inhibitory regions would likely result in a decrease in reward activation in these fROIs, which was not the case in our study. Following this line of reasoning, and in light of the research presented in the previous paragraph relating activity in the dorsal PFC to reward, we would argue that in our study the activity in this regions can best be interpreted in terms of reward processing. Our association of the dlPFC with reward is corroborated by recent research, which showed that the role of the dIPFC may be heterogenous so that it may compute different signals in different contexts (Hutcherson et al., 2012).

In the unbiased viewing condition, however, findings contradicted our hypothesis. There were two patterns of activation, the first of which was observed in brain regions associated with reward: the pulvinar of the right thalamus, dorsal striatum, insula, dorsal and rostral ACC, caudal PCC and anterior PFC. In these reward-associated regions, the pattern of activation was suggestive of an increased reward value of palatable foods in healthy-weight as compared to overweight participants, which directly contradicts our hypothesis. Most of these regions have been shown to be active in response to food or food pictures versus control stimuli: the dorsal striatum (Malik, McGlone, Bedrossian, \& Dagher, 2008; Rothemund et al., 2007; Small, Jones-Gotman, \& Dagher, 2003; 
Small, Zatorre, Dagher, Evans, \& Jones-Gotman, 2001; Stoeckel et al., 2008), caudal PCC (Killgore et al., 2003; Nummenmaa et al., 2012), regions near our fROI in the anterior PFC (Holsen et al., 2005; Nummenmaa et al., 2012), and middle insula (Van der Laan et al., 2011). The middle insula is very often co-active with another fROI, the ACC (Craig, 2009), a region involved in hedonic processing (Kringelbach, 2009). The remaining fROI with this activation pattern, the pulvinar, is considered to be the interconnection of the majority of these fROIs, that is the insula, PCC, ACC and PFC (Mufson \& Mesulam, 1984; Pessoa \& Adolphs, 2010). The function of the pulvinar seems to be the coordination of cortical networks during evaluation of the biological significance of affective visual stimuli (Pessoa \& Adolphs, 2010).

The second pattern of activation of the unbiased viewing condition was suggestive of increased inhibition-related brain activity in overweight as compared to healthyweight participants. This was the case for the anterior frontopolar cortex, lateral frontopolar cortex and frontal eye fields in the anterior dmPFC, which are usually considered regions involved in inhibition or attention-direction (Boorman et al., 2009; Coutlee \& Huettel, 2012; Greenberg et al., 2010; Muggleton et al., 2010).

Taken together, the results of the unbiased viewing condition support the idea that being overweight is associated with a mismatch between inhibitory and reward processes which, in normal eating, are assumed to act in an orchestrated fashion to maintain the balance between food intake and energy expenditure (García-García, Narberhaus, et al., 2013; Metcalfe \& Mischel, 1999). Results in the unbiased viewing condition showed that inhibitory regions were more active and reward regions were less active in the overweight compared with the healthy-weight participants. Apparently these inhibitory processes played a dominating role during unbiased viewing of palatable versus neutral food cues, as this study showed. However, restrained eaters can easily become disinhibited by depressive symptoms (Roefs et al., 2012) or distraction (Boon, Stroebe, Schut, \& Ijntema, 2002), leading to infraction of restrictive intake norms and to overconsumption (Herman \& Polivy, 2007). Further, resources might become depleted over time by chronic self-regulation so that palatable, and often high-calorie, snack food becomes irresistible (Hofmann, Rauch, \& Gawronski, 2007; Vohs \& Heatherton, 2000). Inhibitory neural responses seem to be natural not only in overweight people, but also in successful dieters: successful dieters showed a greater activation than non-dieters after consumption of a meal in the dorsal PFC (DelParigi et al., 2007), in an inhibitory region very near to our fROI in anterior dmPFC. The reason why successful dieters succeed to restrain themselves, in contrast to overweight people, although they engage similar brain regions to inhibit their appetitive responses, remains to be investigated.

The question remains why we found an activation of inhibitory control regions and a lack of activation of reward regions in overweight participants during unbiased viewing, whereas the majority of studies on food reward with a condition of passive 
viewing without instructions found the opposite, that is, more activation in reward regions in overweight compared with healthy-weight participants (e.g., Bruce et al., 2010; Martens et al., 2013; Murdaugh, Cox, Cook, \& Weller, 2012; Rothemund et al., 2007; Stoeckel et al., 2008). The reason could be the type of design that was used: an eventrelated design in our study versus a blocked design in the previous studies. In a blocked design in which multiple palatable food stimuli are shown consecutively, craving for them could be stronger than in an event-related design in which, in our case, the stimuli of different categories (of varying palatability, high-calorie or low-calorie) were mixed. A blocked design may cause a stronger build-up of craving because of the grouped presentation of high-calorie palatable foods, even in a condition of passive viewing without instructions. Such an indulgence would of course overrule any natural inhibitory response, which therefore may be the reason that no such responses were found in the majority of food reward studies.

In conclusion, this study showed that overweight participants are more responsive to variations in palatability (palatable vs. neutral) than healthy-weight participants, in that the difference in activation in response to palatable versus neutral food stimuli was larger in overweight than in healthy-weight participants. However, this was only the case in a condition in which participants were required to focus on the taste of the presented food stimuli. In a condition of unbiased viewing without instructions the opposite was found, that is, reward-related brain activation was reduced in overweight participants, whereas control-related brain activation was increased in overweight participants, both as compared to healthy-weight participants. So, when not specifically focused on taste, inhibitory brain responses are dominant in overweight people. Apparently, this inhibition is easily overridden under disinhibiting circumstances (Boon et al., 2002; Herman \& Polivy, 2007; Roefs et al., 2012), and then they find themselves in a situation in which it is difficult not to think of food in terms of its palatability. Such a situation might be as straightforward as being confronted with an advertisement or commercial for a snack food. The ease with which reward-related brain activation then becomes dominant in overweight/obese persons may be related to their energy depleting attempts to restrain themselves (Hofmann et al., 2007; Vohs \& Heatherton, 2000).

\section{ACKNOWLEDgements}

We would like to thank Ing. Charlie Bonnemayer of Maastricht University for his help in programming the stimulus protocol in E-Prime and Dr. Armin Heinecke of Brain Innovation B.V. for his help with the data analysis. This study was financed by the Netherlands Research Organization (NWO) grant number 400.06.148, awarded to Prof. Dr. Anita Jansen. 



\title{
Chapter 4
}

\author{
THE CRAVING STOPS BEFORE YOU FEEL IT: \\ NEURAL CORRELATES OF CHOCOLATE CRAVING \\ DURING CUE EXPOSURE \\ WITH RESPONSE PREVENTION
}

Frankort, A., Roefs, A., Siep, N., Roebroeck, A., Havermans, R., \& Jansen, A. (in press). The Craving Stops Before You Feel It: Neural Correlates of Chocolate Craving

During Cue Exposure with Response Prevention. Cerebral Cortex. doi:10.1093/

cercor/bht016 


\begin{abstract}
Cue reactivity and craving can be influenced by cue exposure with response prevention (CERP). This study investigated the neural correlates of CERP using fMRI while participants smelled chocolate (17 participants) or a control object (17 participants). CERP was interrupted by 7 scanning sequences measuring the brain response to neutral and chocolate pictures. Chocolate craving was hypothesised to be mirrored by activation in brain reward regions. As expected, control group craving remained similar throughout the session. A short exposure (30 min) increased chocolate craving in the experimental group, which was mirrored by significant group differences in activation in brain reward regions. Unexpectedly, a long exposure $(60 \mathrm{~min}) \mathrm{did}$ not lead to craving extinction in the experimental group, although craving started to decrease at this point. On a neural level however, activation in regions of interest in the experimental group seemed to have extinguished after the long exposure, as activation levels returned to or fell below control group levels. These results indicate that brain reward activation during CERP is linked to craving, at least for a short exposure. Regarding a longer exposure, the decline in brain reward activation in the experimental group may be a precursor of a decrease in craving.
\end{abstract}




\section{INTRODUCTION}

Eating behaviour and food cravings do not only depend on internal sensations of hunger and satiety; they are also controlled by external cues. A revealing example is a study with people suffering from amnesia who, after having had lunch, forgot about having eaten and readily continued eating when served a second and even a third lunch a short time later (Higgs, Williamson, Rotshtein, \& Humphreys, 2008; Rozin, Dow, Moscovitch, \& Rajaram, 1998). This research showed that the simple presence of a meal induces food intake and apparently overrides any satiety signal if one cannot remember that one has recently eaten. Similarly, participants ate considerably more soup when it was served in a bottomless bowl, which concealed the amount of soup that they already had consumed, instead of a normal bowl (Wansink, Painter, \& North, 2005). Apart from food cues such as the presence of a meal or the lacking information about the consumed amount of soup, there are many more cues that promote food intake. These cues include environmental context (Boggiano, Dorsey, Thomas, \& Murdaugh, 2009), food variety (Guerrieri, Nederkoorn, \& Jansen, 2008; Remick, Polivy, \& Pliner, 2009), advertisements (Harris, Bargh, \& Brownell, 2009) and intake of other people (Herman et al., 2003; Hermans, Larsen, Herman, \& Engels, 2012). These and other studies (for a review see Jansen et al. 2011) show that food cues increase the likelihood of food intake and that cue-elicited eating easily leads to overeating and weight gain. Therefore it is of specific interest to find ways of reducing the appetite enhancing influence of food cues. This study will investigate the neural correlates of craving during an intervention that aims to reduce this influence: cue exposure with response prevention (CERP).

Food cues are known to elicit reactivity. Physiological responses reflecting food cue reactivity are insulin release and increased salivation (Jansen et al., 2011). These physiological responses are supposed to be subjectively experienced as craving, which is best described as a strong desire for a given food. In its turn this promotes consumption (Jansen, 1998). This cue-elicited food craving is thought to reflect a learning history. According to the classical conditioning model of binge eating proposed by Jansen (1998), food cues function as a conditioned stimulus (CS) associated with food intake (US). The more experience one has had with the consumption of a particular food, the stronger the reactivity to a cue associated with that food. Thus, overconsumption may contribute to greater cue reactivity, which in turn might facilitate overeating. Indeed, in overweight adults, overweight children and binge eaters, reactivity to food cues was greater than in healthyweight participants (Ferriday \& Brunstrom, 2011; Jansen et al., 2003; Sobik et al., 2005). In obese participants, neural activation in response to high-calorie food versus control pictures in regions associated with food reward was negatively correlated with success in achieving and maintaining weight loss after a weight-loss treatment (Murdaugh et al., 2012). A study with adolescent girls showed similar results, in that activation in food reward regions in response to food versus neutral stimuli was positively correlated with 
body mass index (BMI) and, in one of these regions, was predictive of future weight gain (Yokum et al., 2011b). Interestingly, formerly obese successful dieters showed decreased cue reactivity compared with currently obese unsuccessful dieters (Jansen et al., 2010). This suggests that decreased cue reactivity may aid weight-loss maintenance.

A possible way to decrease food cue reactivity is CERP. During food-related CERP, one is continuously exposed for about an hour to cues or contexts that normally predict food intake but eating is not allowed. During this exposure it is learned that the food cue (e.g. the sight, smell, or context of food intake) no longer predicts food intake (US). After a successful CERP treatment, which usually takes several sessions in different contexts, the cue predicts a 'no eating' response and consequently the conditioned response (food cue reactivity and craving) will have been extinguished. In substance abuse, CERP treatment has not always been effective in achieving abstinence (Havermans \& Jansen, 2003), but this may have been due to the methods being largely suboptimal in earlier studies investigating CERP in drug addiction (e.g., using only one extinction context, which reduces extinction generalizability and therefore increases relapse probability) (Conklin \& Tiffany, 2002). A more recent study (Vollstädt-Klein et al., 2011) did successfully show that a CERP training led to a reduction in neural cue reactivity to alcohol cues in detoxified alcohol-dependent patients, compared with a control intervention. A nicotine CERP intervention using virtual reality also led to a decrease in cue reactivity to smoking cues (Choi et al., 2011). In food studies, data suggest that CERP might be an effective treatment for overeaters: in small-scale pilot studies with binge-eating patients, several sessions of CERP significantly reduced the number of eating binges (Jansen et al., 1989; Martinez-Mallén et al., 2007; Toro et al., 2003). Further, CERP treatment in patients with bulimia nervosa was more effective than a training of self-control techniques (Jansen et al., 1992) or a non-exposure-based intervention in addition to cognitive behaviour therapy (Carter, McIntosh, Joyce, Frampton, \& Bulik, 2006). Although this research has been limited to binge eaters and patients with bulimia nervosa, obese people might profit from CERP treatment as well, since eating binges are prevalent in a considerable number of obese people (B. Bruce \& Agras, 1992; De Zwaan \& Mitchell, 1992). Further evidence of the efficacy of CERP is a study, in which chocolate cravers showed a reduction in cue-induced chocolate craving during a second CERP session with chocolate, compared with the first session, whereas a control group did not show such a reduction (Van Gucht, Vansteenwegen, Beckers, Hermans, et al., 2008). As far as we know, the neural correlates of craving before and after food-related CERP have not yet been investigated, which is therefore the aim of the current study.

Food cravings are accompanied by the activation of brain regions associated with reward processing. Indeed, activation in response to food cues is typically found in the amygdala, hippocampus, insula, caudate, ventral tegmental area and substantia nigra, ventral pallidum, nucleus accumbens and related striatum, as well as in the anterior cingulate, orbitofrontal, prefrontal and posterior fusiform cortices (Appelhans, 2009; Berridge, Ho, Richard, \& DiFeliceantonio, 2010; Berthoud, 2011; Carnell, Gibson, Benson, 
Ochner, \& Geliebter, 2012; Frankort et al., 2012; Kringelbach, 2009; E. T. Rolls, 2011; Small, 2009; Van der Laan et al., 2011). Therefore, we hypothesise that food cue reactivity during a short exposure will be reflected by increased brain activation in the mentioned reward regions, in comparison to a control group exposed to a neutral stimulus. The question arises whether this activation diminishes with the extinction of food craving. To examine this question, a 65 min CERP was conducted with chocolate in healthy-weight women. The chocolate exposure was interrupted intermittently by short brain scans, measuring the response to chocolate and neutral pictures. The chocolate exposure group was compared with a control group who underwent the same procedure except that the controls were exposed to a pencil instead of chocolate.

\section{METHOD}

\section{Participants}

Participants were 34 females (17 in the exposure group and 17 in the control group) of Caucasian ethnicity who were recruited among undergraduate students from Maastricht University willing to participate in research. Only right-handed healthy-weight participants who had a low score (total score $<14$ ) on the Restraint Scale (Herman \& Polivy, 1980) and who were not currently on a weight-loss diet were selected. Further exclusion criteria were a self-reported history of mental disorder or family history of eating disorders. The groups did not differ regarding age, hunger, restraint or trait chocolate craving. However, the groups did tend to differ in BMI, $p=.06$, with the experimental chocolate exposure group being heavier than the control pencil exposure group, while at the same time all participants had a healthy weight $(\mathrm{BMI}<24.9)$ except for one slightly overweight participant in the experimental group $(\mathrm{BMI}=26.0)$. Participant characteristics are shown in Table 1. The datasets of seven additional participants had to be discarded due to scanner problems or excessive head movement. Remuneration for participation was $€ 30$ or course credits. The study was approved by the local ethics committee.

\section{Assessment}

Momentary chocolate craving. Momentary chocolate craving was measured repeatedly during the scanning session on a visual analogue scale (VAS) asking "how much do you crave chocolate at this moment", ranging from 0 (not at all) to 100 (very much).

Trait chocolate craving. The craving subscale of the Attitudes to Chocolate Questionnaire (Benton et al., 1998) was used to assess the trait preoccupation with chocolate and the degree of compulsive behaviour that is elicited by (thinking of) chocolate. The craving score was the mean of ten VAS items (0 to 100) of the subscale, containing questions 
Table 1. Characteristics and self-report data of participants

\begin{tabular}{|c|c|c|c|c|c|c|}
\hline & \multicolumn{2}{|c|}{$\begin{array}{l}\text { Experimental group } \\
\qquad(\mathrm{n}=17)\end{array}$} & \multicolumn{2}{|c|}{$\begin{array}{l}\text { Control group } \\
\quad(\mathrm{n}=17)\end{array}$} & \multirow[b]{2}{*}{$t(32)$} & \multirow[b]{2}{*}{$p$} \\
\hline & $M$ & $S D$ & $M$ & $S D$ & & \\
\hline Age (yrs) & 19.8 & 1.4 & 20.2 & 1.5 & 0.89 & .38 \\
\hline Body mass index & 22.8 & 1.7 & 21.6 & 1.8 & 1.94 & .06 \\
\hline $\begin{array}{l}\text { Time between lunch and scan } \\
(\mathrm{min})\end{array}$ & 86.5 & 14.4 & 82.6 & 20.9 & 0.62 & .54 \\
\hline Hunger $^{1}$ & 18.6 & 14.5 & 14.9 & 14.1 & 0.74 & .46 \\
\hline Restraint $^{2}$ & 10.6 & 2.6 & 10.8 & 4.7 & 0.18 & .86 \\
\hline Trait chocolate craving ${ }^{3}$ & 38.3 & 19.1 & 35.0 & 13.2 & 0.58 & .56 \\
\hline
\end{tabular}

${ }^{1}$ Scored on $100 \mathrm{~mm}$ VAS scales, ranging from 0 (not hungry at all) to 100 (very hungry).

${ }^{2}$ Scored on the Restraint Scale (Herman \& Polivy, 1980), with a minimum total score of 0 (no restraint) and a maximum of 35 (high restraint).

${ }^{3}$ Scored on the craving subscale of the Attitudes to Chocolate Questionnaire (Benton, Greenfield, \& Morgan, 1998), with a minimum total score of 0 (no trait chocolate craving) and a maximum of 100 (high trait chocolate craving).

about chocolate preoccupation, with the scale for each question ranging from 0 (this does not apply to me at all) to 100 (this very much applies to me). The subscale was found to be valid and reliable (Cramer \& Hartleib, 2001; Müller, Dettmer, \& Macht, 2008).

Hunger. Hunger was measured on a VAS: "How hungry do you feel at this moment?", ranging from 0 (not hungry at all) to 100 (very hungry).

Restraint. The Restraint Scale (Herman \& Polivy, 1980) measures the participant's intention to restrain her food intake, her concern about body weight and weight fluctuations. The minimum and maximum total scores are 0 and 35, respectively. Higher scores reflect more intentions to restrain and increased difficulty of controlling food intake. The scale was found to be sufficiently valid and reliable (Scagliusi et al., 2005; Van Strien et al., 2007; Williamson et al., 2007).

Bogus chocolate taste test. A bogus chocolate taste test, in which the actual consumption of chocolate was measured, was conducted after the scanning session. Data of this test will be published in a forthcoming paper.

\section{Experimental Design}

The experimental design was a mixed design comparing an experimental group (exposure to chocolate) with a control group (exposure to a control stimulus), with repeated measures of the brain response to chocolate pictures (when compared with neutral control pictures) and of the momentary craving for chocolate. 


\section{Stimuli}

Stimuli were 56 pictures of chocolate and chocolate products and 56 neutral pictures of office supplies and utensils not related to food. Chocolate pictures did not have any festive associations (such as Valentine, Easter and birthday). The majority of stimuli were purchased online (www.istockphoto.com). Stimuli were presented as pop-out figures on a light grey background in the centre of the screen, covering a visual angle of approximately $12^{\circ}$. For statistical power reasons and to reduce habituation effects, the stimuli were assigned to two separate picture pools. The first picture pool contained 24 chocolate and 24 neutral pictures that were shown in the most crucial runs at the beginning, the peak and the end of the scanning session (runs 1, 5 and 7, respectively). These runs were used for between-group comparisons. Thus, within each of the mentioned three runs, some pictures of this pool were shown twice, but in the course of the whole scanning session, each picture from this pool was shown four times. The second picture pool, intended to measure the time course of neural craving correlates in the chocolate exposure group, contained 32 chocolate and 32 neutral pictures and was used in the remaining runs 2, 3, 4 and 6. In this pool also, each picture was shown four times in the course of each scanning session.

\section{Stimulation Protocol}

The stimuli were presented in a blocked design with a block duration of approximately ${ }^{1}$ $15 \mathrm{~s}$ using E-Prime version 2.0.8.90 (PST, 1996). Blocks contained either eight chocolate or eight neutral pictures, with each picture being presented during $1850 \mathrm{~ms}$ and chosen randomly without replacement from the relevant picture pools. To maintain the participants' attention, half of the stimulus blocks were followed by a question block with a duration of $3 \mathrm{~s}$ (see Figure 1). In this block the participant was asked to indicate whether the depicted picture was presented in the preceding stimulus block. The response was given by means of a button press. All blocks were preceded by a black fixation cross on a light grey background with a duration of $12 \mathrm{~s}$ (before stimulus blocks) or $6 \mathrm{~s}$ (before question blocks). Additionally, after the last block a fixation cross was shown for $12 \mathrm{~s}$ to allow for recording of the remainder of the blood oxygen level dependent (BOLD) response.

One run consisted of four chocolate blocks, four neutral blocks, and four question blocks. For each of seven runs, the order of stimulus blocks was randomised with the constraint that no more than two stimulus blocks of the same category occurred subsequently. Then the question blocks were inserted randomly with the constraint that

1 Some blocks in the first two runs were presented up to $15.1 \mathrm{~s}$, probably due to the time it took E-Prime to retrieve the pictures from the local harddisk for the first time and write them to the cache. Blocks always started with reception of a trigger pulse indicating the MRI scanner's radiofrequency pulse to synchronise with image acquisition. 
they had to follow a chocolate block twice and a neutral block twice. Thus, seven run orders were determined, fixed for all participants. The presentation order of these seven run orders was randomised separately for each participant. The duration of a run was on average $270 \mathrm{s.}^{2}$ Between runs, the participant remained in the scanner and was instructed to hold and smell the object (a piece of chocolate or a pencil) that was handed to her for the exposure duration. This exposure duration increased over time and amounted to 1 min after runs 1 and 2, 2 min after run 3, 3 min after runs 4 and 5, and 20 min after run 6 (see Figure 1).

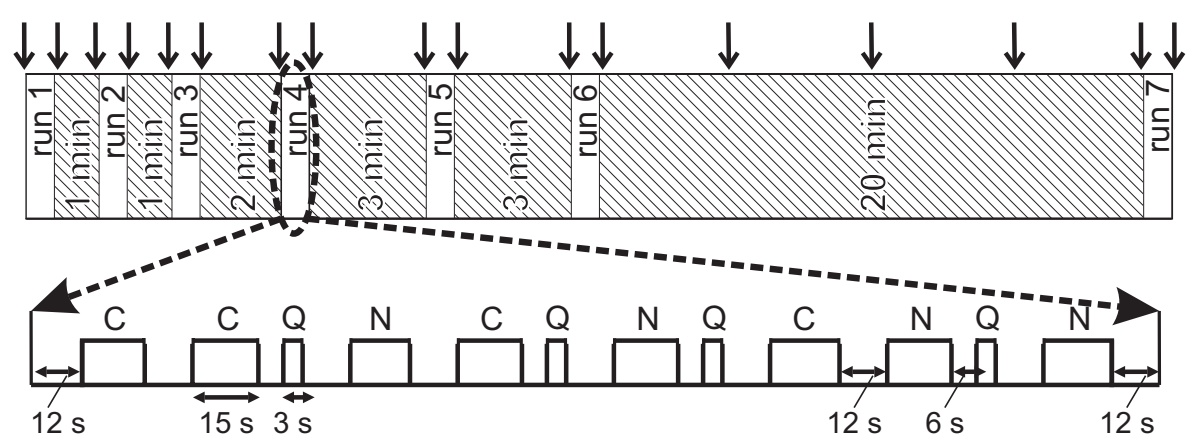

Figure 1. Session protocol with seven scanning runs (white bars), exposure (hatched pattern), and measurements of current chocolate craving (downward arrows). Two different picture pools were used for runs 1, 5 and 7 and for runs 2, 3, 4 and 6. In the enlargement an example of a stimulation protocol of one run is shown, consisting of four chocolate blocks (C), four neutral blocks $(\mathrm{N})$, and four question blocks $(\mathrm{Q})$. Stimulus blocks had a duration of approximately $15 \mathrm{~s}$ and were preceded by fixation times of $12 \mathrm{~s}$. Question blocks had a duration of $3 \mathrm{~s}$ and were preceded by fixaton times of $6 \mathrm{~s}$.

\section{Session Protocol}

The length of the session was 65 min, during which CERP was conducted with real chocolate (experimental group) or with a control stimulus (control group) in healthyweight women, while the olfactory exposure was interrupted intermittently by seven short brain scans (see Figure 1). During the brain scans, there was no exposure to real chocolate; only the response to both chocolate pictures and neutral pictures was measured. The session duration of approximately one hour was chosen due to results of CERP treatment studies (Jansen et al., 1989; Toro et al., 2003), in which the subjective ratings of cue reactivity as measured in the first session increased directly after the

2 The average run duration was longer than the anticipated $264 \mathrm{~s}$ because some of the trigger pulses were missed, leading to prolongation of fixation times between blocks, as waiting times for trigger pulses between blocks were filled with fixation. 
start of the exposure, peaked after 10 to $30 \mathrm{~min}$, decreased thereafter and finally extinguished. The session started with a functional run and was then followed by exposures and functional runs in an alternating order. Exposure durations were initially short and increased with increasing time, amounting to 1, 1, 2, 3, 3, and 20 min respectively. The final prolonged exposure duration was long to attain an extinction of craving.

For participants in the experimental group, the exposure was done with real chocolate. Chocolate was chosen as a cue to perform CERP in healthy participants, because it is the food most frequently craved by Western women (Pelchat, 1997). During each exposure cycle, the participant held a piece of chocolate (approximately $4 \mathrm{~cm} \times 2$ $\mathrm{cm} \times 1 \mathrm{~cm}$ ), the lower part of which was wrapped in an odourless tissue, under her nose. This was handed to her by the experimenter at the beginning of each exposure, and was given back to the experimenter at the end of each exposure. Therefore, during functional runs there was no exposure to real chocolate. As there were several types of chocolate available (eight different brands of milk and dark chocolate), the participant was instructed to ask for a new piece of chocolate as soon as she had become habituated to the smell of the current one. In practice, this resulted in a replacement of chocolate at least every few minutes. At the beginning and at the end of every functional run the participant rated momentary chocolate craving on a VAS by means of a joystick placed on her abdomen. Additionally, in the final prolonged exposure cycle of 20 min duration, momentary chocolate craving was assessed every five minutes. The control group procedure was the same as for the experimental group, the only difference being that the participant had to sniff at one of eight available pencils instead of chocolate. After the last functional run there was one anatomical measurement.

\section{Procedure}

There was one afternoon scanning session per participant. Participants were requested to refrain from eating chocolate products and from drinking coffee or tea on the scanning day, as the brain response is influenced by caffeine (Koppelstaetter et al., 2010). In addition, they had to consume a regular lunch 1 to 1.5 hours before the start of the scanning session. Upon arrival, the participant's subjective hunger ratings, together with restraint and trait craving for chocolate were assessed. Additionally, her weight and height were measured. Then the participant entered the scanner and the scanning session started. After the scanning session, the bogus chocolate taste test was done in an adjacent room. After filling in an exit form with questions about what she mainly had been thinking about while viewing chocolate and neutral pictures, the participant was thanked and compensated for participation. Debriefing was done by email at the end of the study. 


\section{fMRI Data Acquisition}

All images were acquired with a 3T Siemens Magnetom Allegra Head-only scanner. The head coil encompassed a small mirror, through which participants could see the stimuli that were projected on a screen at the head end of the scanner. Gradient-echo planar imaging (EPI) parameters were optimised (Deichmann et al., 2003; Weiskopf et al., 2007) to acquire functional volumes ( 50 slices, $\mathrm{TR}=3000 \mathrm{~ms}$ ) with reduced susceptibility and distortion artefacts in the OFC. These settings included a TE of $25 \mathrm{~ms}$, oblique axial slices acquired in interleaved order with a negative (i.e. backward) tilt angle of $30^{\circ}$, a voxel size of $2 \mathrm{~mm} \times 2 \mathrm{~mm} \times 2.5 \mathrm{~mm}$, a field of view (FoV) of $256 \times 192 \mathrm{~mm}^{2}$, an imaging bandwidth of $2790 \mathrm{~Hz}$ over FoV, an echo spacing of $0.42 \mathrm{~ms}$, and flip angle $90^{\circ}$, resulting in $\mathrm{T} 2{ }^{*}-$ weighted images. There were 88 volumes per functional run. After the last functional run, an optimised MP RAGE sequence was used (Deichmann et al., 2000; Mugler \& Brookeman, 1990) with the following settings: $\mathrm{TR}=2250 \mathrm{~ms}, \mathrm{TE}=2.6 \mathrm{~ms}$, flip angle $=9^{\circ}$, voxel size $1 \mathrm{~mm} \times 1 \mathrm{~mm} \times 1 \mathrm{~mm}$, echo spacing $6.9 \mathrm{~ms}$, resulting in a high-resolution T1-weighted anatomical scan for co-registration.

\section{fMRI Data Preprocessing}

BrainVoyager QX version 2.4.1.2052 (BrainInnovation, 2001) was used for analysis. Due to T1 saturation effects, the first two volumes of each functional run were excluded. Preprocessing consisted of slice scan time correction with cubic spline interpolation, removal of low-frequency noise using high-pass temporal filtering $(0.0075 \mathrm{~Hz}$ cut-off $)$, and threedimensional motion correction using trilinear interpolation for alignment and sinc interpolation for final resampling. Subsequently, preprocessed data were co-registered with the anatomical scan, resulting in co-registered three-dimensional space data over the course of time for each run, with a functional voxel resolution of $2 \mathrm{~mm} \times 2 \mathrm{~mm} \times 2 \mathrm{~mm}$. All data were spatially normalised using Talairach transformation procedures (Talairach \& Tournoux, 1988) and finally spatially smoothed with a $6 \mathrm{~mm}$ full-width-at-half-maximum isotropic Gaussian Kernel. For group-level analyses, Talairach-standardised anatomical data sets of participants were averaged, based on which a whole-brain mask was generated.

\section{Data Analysis}

Momentary chocolate craving scores were analysed in a 2 (group: exposure vs. control) $\times 3$ (time of measurement: prior to run 1 vs. prior to run 5 vs. prior to run 7) analysis of variance (ANOVA). Only these three runs were entered into the ANOVA, to keep the analyses of craving scores and brain data similar. For fMRI data, boxcar predictors were set for chocolate, neutral, and question blocks. Question block predictors were regarded as 
of no interest (confounds). All predictors were convolved with a standard hemodynamic response function (Friston et al., 1998). To optimise detection power, only runs 1 (first run), 5 (the middle of the session and the expected peak of craving in the exposure group) and 7 (last run) were entered into the general linear model (GLM). Z-transformed motion correction parameters were added, resulting in the GLM design matrix. In this modelling approach, the response to fixation times between blocks can be considered as the baseline.

Brain responses in run 5 to chocolate and neutral pictures were analysed in a 2 (group: exposure vs. control) $\times 2$ (picture type: chocolate vs. neutral) random-effects analysis of variance (ANOVA). Because we were interested in brain regions specifically reacting to cue exposure, we looked for an interaction between group and picture type in run 5 (the middle of the session), as the peak of the craving curve in the exposure group was expected to occur in this run, whereas no such peak was expected in the control group. Therefore a whole-brain statistical $F$ map was created containing voxels with a significant interaction in run 5 between group and picture type. Voxel clusters consisting of at least 27 contiguous voxels, each with a $p$ value of interaction below 0.01 (uncorrected), were considered a functional region of interest (fROI). This minimal cluster size was determined with a tool in BrainVoyager QX that performs a cluster-level correction of multiple comparisons at $p=0.05$ by using a Monte Carlo simulation based approach (Forman et al., 1995; Goebel et al., 2006) with 1000 iterations and a voxel size of $2 \mathrm{~mm} \times 2$ $\mathrm{mm} \times 2 \mathrm{~mm}$. The Talairach-standardised whole-brain mask contained 191443 voxels of this size. Thus, at the uncorrected level of 0.01 , only clusters larger than $216 \mathrm{~mm}^{3}$ were considered as fROIs. An anatomical localization indication of the fROIs was obtained with the Talairach Client (www.talairach.org) (Lancaster et al., 2000).

A stricter test of our hypothesis would be the identification of brain regions with a three-way interaction between brain activation over time, in response to both picture categories and in both groups. Therefore, brain responses to chocolate and neutral pictures were additionally analysed in a 2 (group: exposure vs. control) $\times 3$ (time of measurement: run 1 vs. run 5 vs. run 7 ) $\times 2$ (picture type: chocolate vs. neutral) three-way random-effects ANOVA. This analysis yielded a whole-brain statistical $F$ map consisting of voxels with a significant interaction ( $p<0.01$ (uncorrected)) of the factors group, time of measurement and picture type. The minimal cluster size for this analysis, as determined by the BrainVoyager QX tool mentioned before, was found to be 37 functional voxels $\left(296 \mathrm{~mm}^{3}\right)$.

For the second-level analysis in SPSS version 18, average $\beta$ values were extracted for each fROI, run, picture type and participant. With these $\beta$ values, independent samples $t$ tests of the response to chocolate minus neutral pictures were performed for the three runs that were designed for between-group comparisons (runs 1, 5 and 7). Additionally, the significance of activation changes per group over the course of time was calculated with paired samples $t$ tests. 


\section{Results}

\section{Momentary chocolate craving}

As expected, a short (30 min) chocolate exposure led to increased craving compared with the baseline, more so than exposure to a control stimulus (see Figure 2). This was indicated by a significant group $\times$ time effect in a 2 (group: exposure vs. control) $\times 3$ (time of measurement: prior to run 1 vs. prior to run 5 vs. prior to run 7 ) ANOVA, $F(2,31)=7.10$, $p=.003$. This interaction effect qualified a significant main effect of time, $F(2,31)=8.19$, $p=.001$. Craving scores did not differ between groups at baseline, $t(32)=.35, p=.73$, but did differ in the middle of the session (prior to run 5 ), $t(32)=2.62, p=.01$, and at the end of the session (prior to run 7 ), $t(32)=2.90, p=.007$. Looking at the chocolate craving ratings of all six exposure durations, significant group differences were found prior to runs $4,5,6$ and 7, as well as during the final prolonged exposure. Interesting is the progress of craving before and after run 7: the highly significant $(p<.01)$ group difference prior to this run disappeared $(p=.09)$ after the brain scan.

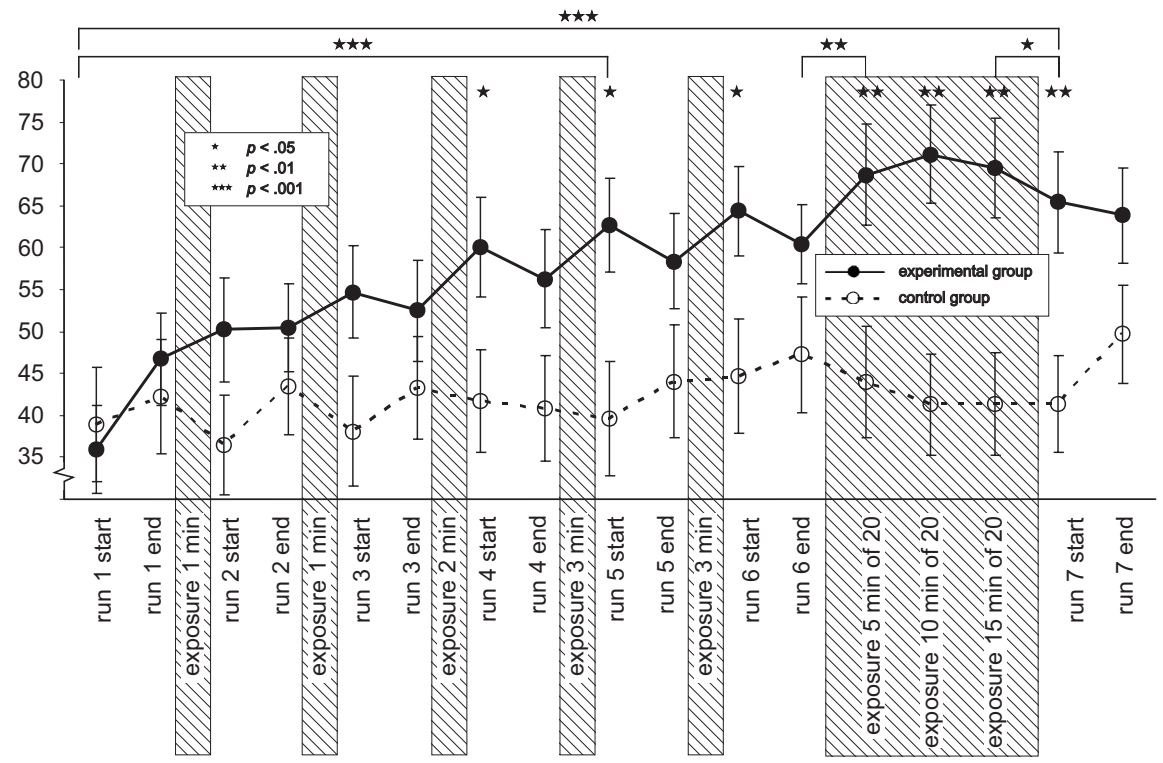

Figure 2. Momentary chocolate craving, as indicated by self-report on a $100 \mathrm{~mm}$ VAS scale asking "How much do you crave chocolate at this moment", ranging from 0 (not at all) to 100 (very much), averaged over groups. Significance brackets correspond to within-group differences in the chocolate exposure group. Asterisks without brackets indicate significant between-group differences. Paired $t$ tests were performed only over craving levels prior to runs 1, 5 and 7 as well as over subsequent measurement time points of the final long exposure. Reported $t$ tests are uncorrected. X-axis is not to scale in regard to time passed. 
Within the chocolate exposure group, craving levels prior to runs 5 and 7 were significantly different from the baseline, $t(16)=6.03, p<.001$ and $t(16)=4.99, p<.001$, respectively (see Figure 2). No difference was found between craving in the middle of the session (prior to run 5 ) and at the end of the session (prior to run 7$), t(16)=.84, p=.42$. This demonstrates that the short (30 $\mathrm{min}$ ) chocolate exposure was successful in increasing craving in the exposure group, but that, contrary to our hypothesis, the long (60 $\mathrm{min}$ ) exposure did not lead to an extinction of craving. In the control group no significant differences in momentary chocolate craving prior to runs 1, 5 and 7 were found, which confirmed our expectations.

The six individual chocolate exposures were each successful in increasing craving in the exposure group, as the average of the six post-exposure craving ratings in this group was significantly higher than the average of the six pre-exposure craving ratings, $t(16)=3.57$, $p=.003$. In the control group, the opposite was found: the average of the six post-pencilexposure ratings of momentary chocolate craving were significantly lower in this group than the average of the six pre-pencil-exposure craving ratings, $t(16)=2.27, p=.04$.

\section{Brain response}

The less restrictive two-way analysis in which fROIs were based on the brain activation of the peak run yielded nine significantly large fROIs with a significant interaction between group and picture type (see Table 2 and Figure 3, locations and bar plots in orange).

These fROIs were both amygdalae, posterior fusiform gyri in both hemispheres, medial posterior cingulate cortex, a region in right parahippocampal and lingual gyrus, and regions in the left somatosensory cortex, left frontal eye fields (FEFs) and left supplementary motor area (SMA). The first seven of these fROIs are considered to be involved in reward processing (Killgore et al., 2003; Van der Laan et al., 2011) and the last two in inhibitory control and control of attention (Fox et al., 2005). The second-level analysis with $\beta$ values of these fROIs showed two response patterns, coinciding with the presumed function of the respective region: in the regions associated with reward, the activation in run 5 was significantly higher in the chocolate exposure than in the control group (all ps $<.01$ ), indicating a higher reward in the experimental group during the presentation of chocolate pictures versus neutral pictures. In the regions associated with control, this activation was lower in the experimental than in the control group (all $p s<.01$ ), indicating a lower effort of controlling chocolate craving in the former group. In both response patterns the activations in run 1 (before the first exposure) and run 7 (the last run) did not differ between groups.

The three-way analysis of group, time of measurement and picture type resulted in six fROIs larger than the respective minimal cluster size, each with a significant threeway interaction (see Table 3 and Figure 3, locations and bar plots in green). These fROIs were located in the left and right caudate, left striate and bilateral extrastriate cortex, and on the border of the right parahippocampal gyrus with the lingual and posterior cingu- 
Table 2. fROIs resulting from the two-way interaction between group (exposure vs. control) and picture type (chocolate vs. neutral) in run 5 (the middle of the session as well as the expected peak of craving in the exposure group)

\begin{tabular}{|c|c|c|c|c|c|c|c|c|}
\hline \multirow{2}{*}{ 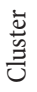 } & \multirow{2}{*}{ size $\left(\mathrm{mm}^{3}\right)$} & \multirow{2}{*}{ Anatomical label } & \multicolumn{6}{|c|}{ Peak voxel values ${ }^{1}$} \\
\hline & & & estimated $\mathrm{BA}^{2}$ & $\mathrm{x}$ & $\mathrm{y}$ & z & $F$ & $p$ \\
\hline \multirow{2}{*}{ A } & \multirow{2}{*}{1024} & peak 1 : amygdala $\mathrm{R}$ & - & 20 & -4 & -14 & 20.04 & 0.00009 \\
\hline & & peak 2 : PHG R & - & 24 & -12 & -15 & 13.61 & 0.00080 \\
\hline \multirow{2}{*}{ B } & \multirow{2}{*}{262} & peak 1: uncus L & 28 & -19 & 0 & -22 & 14.44 & 0.00061 \\
\hline & & peak 2: amygdala L & - & -18 & -4 & -16 & 10.78 & 0.00248 \\
\hline $\mathrm{C}$ & 388 & fusiform gyrus R & 37 & 49 & -54 & -13 & 17.39 & 0.00020 \\
\hline $\mathrm{D}$ & 489 & fusiform gyrus L & 37 & -43 & -41 & -17 & 13.06 & 0.00100 \\
\hline $\mathrm{E}$ & 314 & frontal eye fields L & 8 & -26 & 17 & 49 & 16.92 & 0.00025 \\
\hline $\mathrm{F}$ & 306 & PCC (medial) & 29 & -4 & -48 & 13 & 16.06 & 0.00034 \\
\hline G & 364 & PHG R & 30 & 13 & -38 & -1 & 14.88 & 0.00052 \\
\hline $\mathrm{H}$ & 265 & pre-SMA L & 6 & -44 & 6 & 42 & 13.91 & 0.00074 \\
\hline I & 226 & somatosensory cortex L & 3 & -32 & -31 & 59 & 12.78 & 0.00114 \\
\hline
\end{tabular}

Abbreviations. $\mathrm{BA}=$ Brodmann Area; $\mathrm{L}=$ left hemisphere; $\mathrm{PCC}=$ posterior cingulate cortex; $\mathrm{PHG}=$ parahippocampal gyrus; $\mathrm{R}=$ right hemisphere; $\mathrm{SMA}=$ supplementary motor area.

${ }^{1}$ Voxel coordinates are reported in Talairach space.

'Identified with the "nearest grey matter" option in the Talairach Client (www.talairach.org) (Lancaster et al., 2000).

late gyrus. Between-group $t$ tests of the $\beta$ values of these fROIs showed that during the last run, the chocolate exposure group's activation was significantly lower than that of the control group, whereas in the first run or in the middle of the session this pattern was reversed. While Figure 3 shows the $\beta$ values of the crucial runs 1, 5 and 7 only, supplementary Figure $\mathrm{S} 1$ shows the $\beta$ values of all seven runs of the mentioned fROIs. Because of the quick and direct succession of runs 2, 3 and 4 with the same stimuli, it is likely that habituation has occurred in these runs, which can be seen from decreasing activation from run 2 to 4 in most of the fROIs.

Figure 3 (on next page). Upper part: in orange: $F$ map of clusters larger than $216 \mathrm{~mm}^{3}$ with a significant group $\times$ picture type interaction in run 5 (the expected peak of craving in the experimental group). In green: $F$ map of clusters larger than $296 \mathrm{~mm}^{3}$ with a significant time of measurement $\times$ group $\times$ picture type interaction. $F$ maps were overlayed on a brain averaged over all participants, shown in radiological convention. Lower part: Bar plots of $\beta$ values of clusters shown above, indicating group responses to chocolate pictures minus responses to neutral pictures ( $z$ scores \pm S.E.M.). Bar plots of two inhibition regions, shown with a grey background, have the opposite response of reward regions. Significance indications: ${ }^{\circ} p<.09 ;{ }^{*} p<.05 ;{ }^{* *} p<.01 ;{ }^{* *} p<.001$. Significance brackets correspond to within-group differences. Asterisks without brackets indicate significant between-group differences. Reported $t$ tests are uncorrected. 


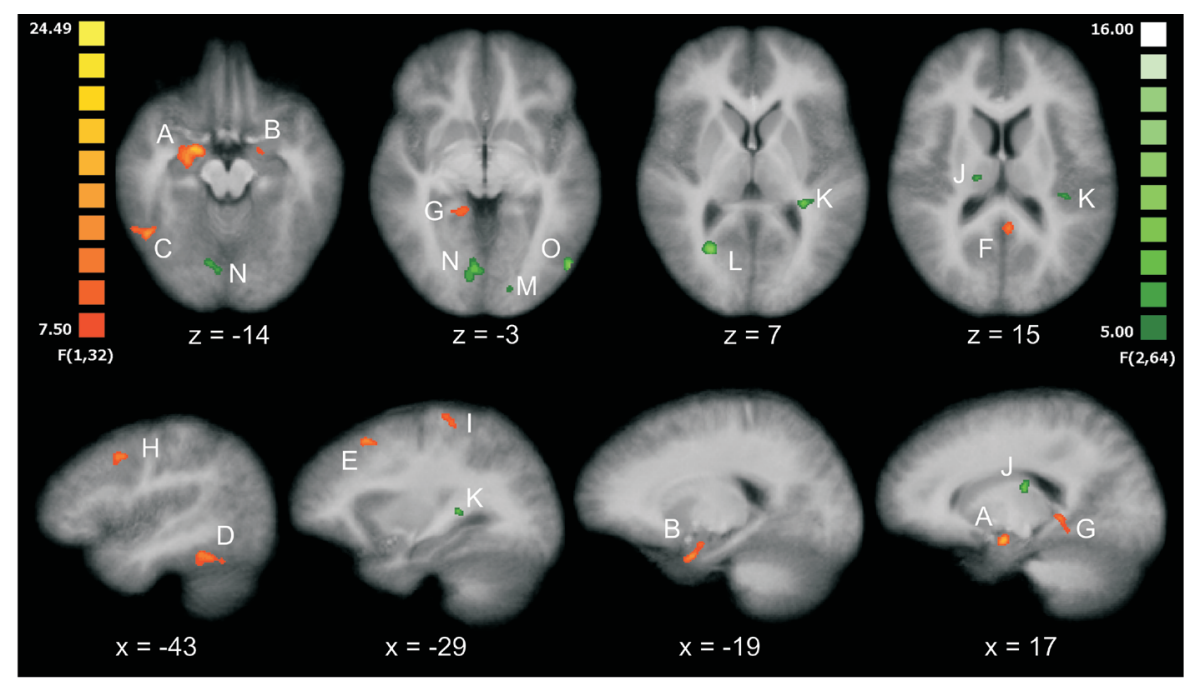

fROls with interaction of group $x$ picture category in run 5 (middle of the session)

experimental group $\square$ control group

fROls with interaction of group $x$ picture category $x$ time of measurement (run 1 vs. run 5 vs. run 7 ) 口 experimental group $\square$ control group

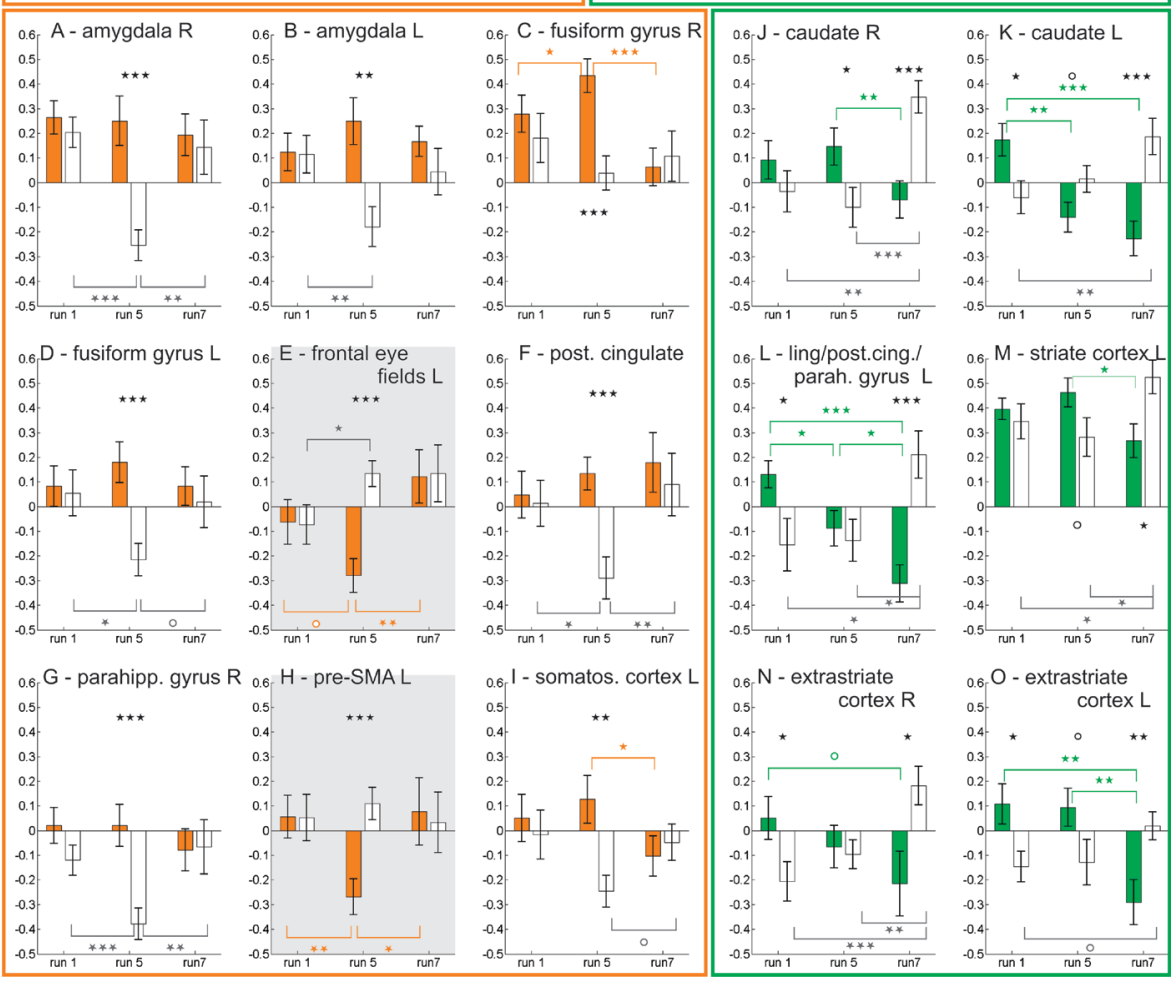


Table 3. fROIs resulting from the three-way interaction between group (exposure vs. control), picture type (chocolate vs. neutral) and time of measurement (run1 vs. run 5 vs. run 7)

\begin{tabular}{|c|c|c|c|c|c|c|c|c|}
\hline \multirow{2}{*}{ 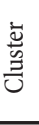 } & \multirow{2}{*}{$\begin{array}{l}\text { size } \\
\left(\mathrm{mm}^{3}\right)\end{array}$} & \multirow[b]{2}{*}{ Anatomical label } & \multicolumn{6}{|c|}{ Peak voxel values $^{1}$} \\
\hline & & & $\begin{array}{c}\text { estimated } \\
\mathrm{BA}^{2}\end{array}$ & $\mathrm{x}$ & $\mathrm{y}$ & $\mathrm{z}$ & $F$ & $p$ \\
\hline $\mathrm{J}$ & 414 & $\begin{array}{l}\text { caudate body R (spreading into } \\
\text { claustrum and thalamus) }\end{array}$ & - & 20 & -19 & 21 & 11.02 & 0.00008 \\
\hline \multirow{2}{*}{ K } & \multirow{2}{*}{306} & peak 1: caudate tail L & - & -30 & -34 & 7 & 8.77 & 0.00043 \\
\hline & & peak 2: posterior insula $\mathrm{L}$ & 13 & -37 & -28 & 13 & 7.36 & 0.00132 \\
\hline $\mathrm{L}$ & 639 & Lingual gyrus, PCC and PHG R & 30 & 25 & -62 & 5 & 11.58 & 0.00005 \\
\hline M & 305 & striate cortex L & 17 & -13 & -93 & -8 & 8.44 & 0.00055 \\
\hline \multirow{2}{*}{$\mathrm{N}$} & \multirow{2}{*}{1334} & peak 1: declive (cerebellum) R & - & 6 & -75 & -11 & 9.14 & 0.00030 \\
\hline & & peak 2: extrastriate cortex R & 18 & 7 & -75 & -5 & 8.68 & 0.00046 \\
\hline $\mathrm{O}$ & 309 & extrastriate cortex L & 19 & -50 & -72 & -3 & 10.19 & 0.00014 \\
\hline
\end{tabular}

Abbreviations. $\mathrm{BA}=$ Brodmann Area; $\mathrm{L}=$ left hemisphere; $\mathrm{PCC}=$ posterior cingulate cortex; $\mathrm{PHG}=$ parahippocampal gyrus; $\mathrm{R}=$ right hemisphere.

${ }^{1}$ Voxel coordinates are reported in Talairach space.

${ }^{2}$ Identified with the "nearest grey matter" option in the Talairach Client (www.talairach.org) (Lancaster et al., 2000).

\section{Discussion}

The current study investigated the neural correlates of craving during CERP, which is an intervention to reduce the appetite-enhancing influence of contextual or food cues on food intake, provided the exposure is long enough (approximately one hour). Note that a short exposure leads to an increase in craving. In participants smelling chocolate or smelling a control stimulus (a pencil), chocolate craving as well as brain activation in response to neutral and chocolate pictures were measured intermittently during the exposure session. In the chocolate exposure group, craving was expected to rise after the start of the exposure session, to peak after approximately $30 \mathrm{~min}$ (the middle of the session, considered a short exposure) and then to decline and extinguish by the end of the session (65 min, considered a long exposure). In the control group, craving was expected to only slightly increase over the course of the procedure, due to the presentation of chocolate pictures during the seven scans. Brain reward activation, which is activation in response to chocolate pictures minus the response to neutral pictures in regions associated with reward, was expected to be concomitant with chocolate craving ratings, that is, to be equal in both groups at the beginning and at the end of the exposure session, and to be higher in the chocolate exposure group than in the control group at the expected peak of the exposure session. 

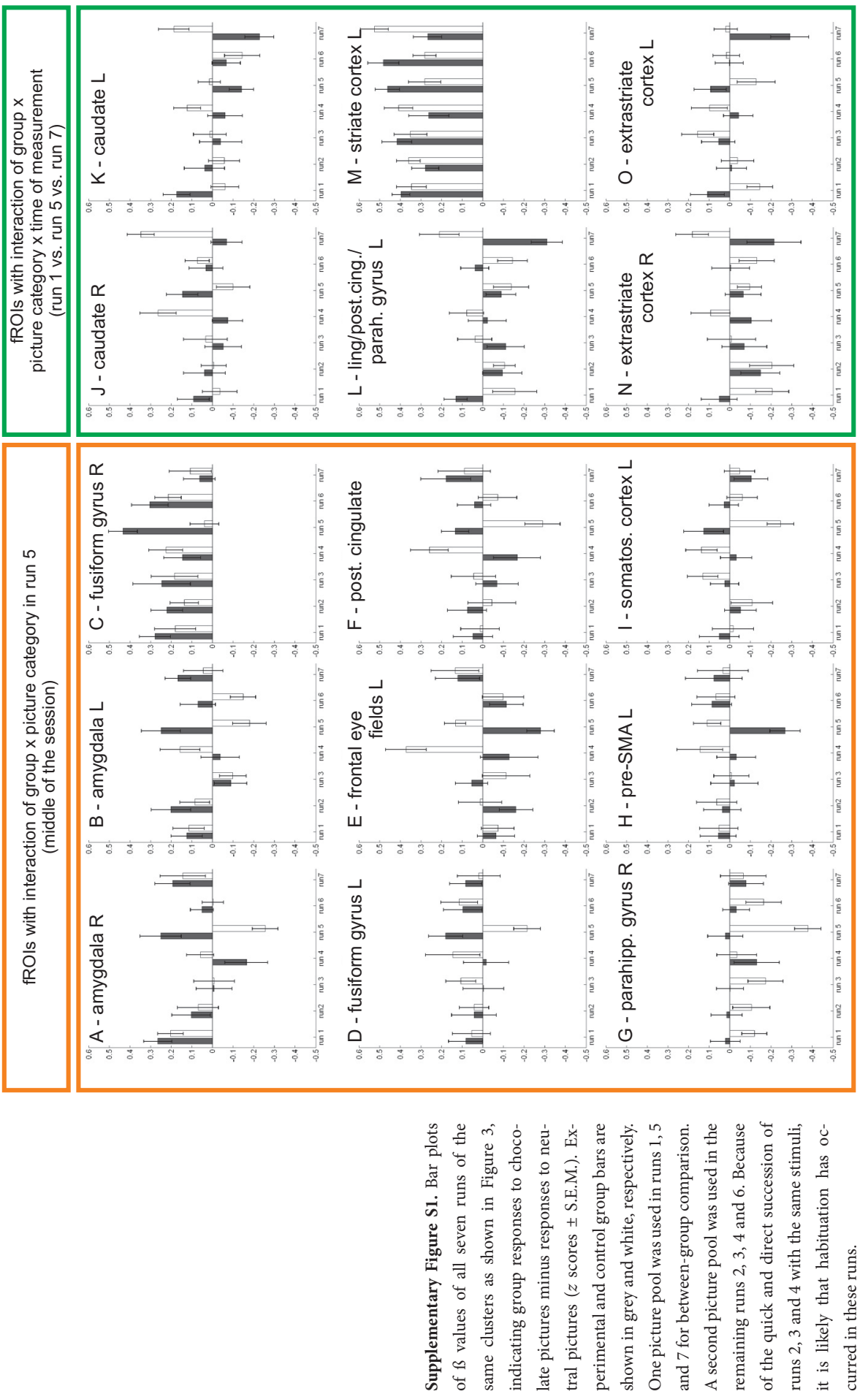
In the chocolate exposure group, craving did rise as expected after the start of the exposure session, but did not decline towards the end of the session. In this group only a small (but significant) decrease in craving was found at the end of the final prolonged exposure. In the control group, craving did not significantly change over the course of the session. Between-group comparisons revealed that there were no group differences at the start of the session (prior to run 1), but that there were significant differences at the expected peak of craving (prior to run 5), which continued to exist until after the final prolonged exposure (prior to run 7). Thus, craving of the chocolate exposure group cannot be considered to have extinguished at the end of the session. Given that the largest between-group difference in craving ratings between the first and the last exposure occurred just prior to run 5 , brain activation analyses were conducted as planned with run 1 (start of the session), run 5 (short exposure, middle of the session) and run 7 (long exposure, end of the session).

Further analyses of craving ratings revealed that the smell of real chocolate (during exposures) was more effective in increasing chocolate craving than the sight of chocolate pictures (during brain scans), as indicated by a higher average of the six craving ratings after as compared with before each exposure in the chocolate exposure group. The opposite pattern in the control group, that is lower average post-exposure craving ratings as compared with pre-exposure ratings, indicated that the sight of chocolate pictures during the scan runs was more effective in increasing chocolate craving than the smell of a pencil. These findings are in line with previous literature, in that perception of a real food cue and mental imagery of that cue overlap with regard to elicited craving (Kavanagh, Andrade, \& May, 2005; Tiggemann \& Kemps, 2005) and habituation to the (imagined) food (Morewedge, Huh, \& Vosgerau, 2010). Our findings in the chocolate exposure group add to this literature by indicating that the perception of the real food is a stronger cue than the image of that food with its accompanying imagined smell and taste.

Regarding brain reward activation, fROIs were first determined by a whole-brain two-way interaction between group and picture type in the middle of the session. This resulted in nine fROIs, all of which have been associated with reward processing in previous studies. In seven fROIs, brain reward activation in the middle of the session was higher in the chocolate exposure group than in the control group, whereas this group difference was absent both at the start and at the end of the exposure session. These seven fROIs have been implicated in encoding the motivational value of food stimuli: bilateral amygdalae (Arana et al., 2003; Gearhardt et al., 2011; Killgore et al., 2003), bilateral posterior fusiform gyri (Frank et al., 2010; Van der Laan et al., 2011), posterior cingulate cortex (Killgore et al., 2003; Rothemund et al., 2007), parahippocampal gyrus (LaBar et al., 2001) and somatosensory cortex (Stice et al. 2011, who call a nearby fROI 'postcentral gyrus'). A higher activation in these regions is considered to represent a higher reward in response to a stimulus or an increase in appetitive motivation; therefore, the 
activation in these fROIs in the middle of the session is in line with our hypothesis. Similarly, in the remaining two of the nine fROIs - the left frontal eye fields and the left pre-SMA - the group difference was absent at the start and at the end of the exposure session; yet here brain reward activation in the middle of the session was lower (instead of higher) in the chocolate exposure group, compared with the control group. This pattern of activation fits with the functionality that has previously been linked with these regions: FEF and pre-SMA have been associated with top-down attentional control and with inhibition of responses (DiQuattro \& Geng, 2011; Fox et al., 2005; Moore \& Armstrong, 2003; Muggleton et al., 2010). Thus, compared with the control group, a lower activation in these regions in the chocolate exposure group is assumed to represent either a lower amount of effort needed to pay attention to the chocolate pictures versus the neutral ones, or a lower degree of craving suppression when seeing these pictures.

When looking in more detail at the activation pattern in appetitive motivation regions with a two-way interaction, it can be seen that the transition from similar levels of activation at the start to significant group differences in the middle of the session originated not only from a rise in activation in the chocolate exposure group, but also from a decline in activation in the control group. This control group decline may be due to habituation to the repeatedly shown visual stimuli, also called repetition suppression, which has also been found in previous studies for the majority of our fROIs involved in appetitive motivation like the amygdala (Weierich, Wright, Negreira, Dickerson, \& Barrett, 2010) and ventral visual stream areas like the posterior fusiform gyri and the parahippocampal gyrus (Vuilleumier et al., 2005; Zweynert et al., 2011). We assume that in the experimental group, repetition suppression is prevented by the exposure to chocolate, considering that the two groups underwent exactly the same procedure except for the cue during the exposures. After the transition from the middle to the end of the session, repetition suppression in the control group seemed to have disappeared, as activation levels in run 7 increased again to a level similar to that of the exposure group. This is probably due to the final prolonged exposure, which lasted $20 \mathrm{~min}$ and took place without being interrupted by any brain scans, so there were no picture presentations during this time span.

In the left pre-SMA, one of the two fROIs with a two-way interaction involved in inhibitory control, the transition from similar levels of activation from the start of the session to significant group differences in the middle of the session originated mainly from a significant decline in activation in the chocolate exposure group. This can be explained by impaired inhibition in response to the chocolate pictures after the chocolate exposure; inhibition has been previously found to be impaired in cue exposure studies with alcohol (Gauggel et al., 2010; Muraven \& Shmueli, 2006). In the second inhibitory control region, the left FEF, the transition from similar levels of activation in run 1 to significant group differences in run 5 was the result mainly from a significant increase in activation in the control group, which as discussed before may represent an increased effort in paying attention to the chocolate pictures (DiQuattro \& Geng, 2011). 
When comparing chocolate craving ratings with brain reward activation levels in fROIs with a two-way interaction, it can be seen that both measures were in accordance at the beginning (no group differences) and in the middle of the session (significant group differences in both measures). However, at the end of the session there was a dissociation between these measures: chocolate craving still differed significantly between groups (although a decline in ratings had started in the chocolate exposure group), whereas brain reward activation levels in the nine identified fROIs returned back to similar levels in both groups. This dissociation could be caused by the tendency of participants to give consistent answers to the same repeatedly asked VAS items (Schubert \& Fiske, 1973; Schubert, 1975). Another explanation for the finding that craving on a neural level had already decreased, at the moment when the subjective report of craving had only started to decrease, could be that brain reward activation levels are a precursor of a decrease in the subjectively experienced craving. After all, before a cue can elicit a subjective feeling, it has to be processed in the brain. This is in line with findings that brain activation accompanying a decision to act occurs prior to the conscious awareness of this decision (Custers \& Aarts, 2010; Libet, Gleason, Wright, \& Pearl, 1983; Soon, Brass, Heinze, \& Haynes, 2008). Therefore, an investigation of the transient subjective and neural response to food in hungry participants who gradually become satiated would shed more light on this supposedly precursory characteristic of the brain.

Results of the stricter three-way analysis corroborate the interpretation of neural craving correlates as a precursor of subjective craving. The activation pattern showed that in all six fROIs with a three-way interaction, activation in run 7 (i.e., after the last prolonged exposure), was lower in the exposure group than it was in the control group. The sign of this group difference before the last prolonged exposure (in run 1 or 5) was reversed (i.e., the exposure group had a higher activation than the control group). Five of the six fROIs with a three-way interaction between group, picture type and time of measurement, have been found to be active in previous studies investigating the response to food or to food pictures versus control pictures. These were the left and right caudate nuclei, very close to locations found by St-Onge et al. (2005), aside from studies that found activation of other parts of the caudate nuclei (Malik et al., 2008; Rothemund et al., 2007; Small, Jones-Gotman, et al., 2003; Small et al., 2001; Stoeckel et al., 2008). Further there was a region in the left striate cortex (Führer, Zysset, \& Stumvoll, 2008; Malik et al., 2008; Rothemund et al., 2007; Tang et al., 2012), as well as two regions in extrastriate cortex (Malik et al., 2008; Rothemund et al., 2007; Schienle et al., 2009). Finally, the sixth fROI on the border of the right lingual gyrus, the posterior cingulate gyrus and the parahippocampal gyrus has been found to have a smaller grey matter volume with increasing participants' BMI in a previous study (Walther, Birdsill, Glisky, \& Ryan, 2009). The fact that the exposure group had a lower activation than the control group in run 7 , combined with findings that most of the fROIs have been implicated in food reward, leads to the interpretation that at the end of the session the exposure group had a lower reward of 
chocolate pictures versus neutral pictures than the control group. Keeping in mind that, at this point in time, the subjective craving reports were still higher in the exposure group than they were in the control group, this is in line with the assumption that neural correlates of craving could be a precursor of subjectively experienced craving. Regarding the activation pattern before the last prolonged exposure, where the group difference was reversed compared with run 7 , this could indicate that the prolonged chocolate exposure was effective in reducing chocolate reward on a neural level, although subjective ratings did not (yet) indicate this. Aside from this, the fact that the exposure group had a higher activation in run 1 in some of the fROIs than the controls could be due the instructions: they had been told before the start of the session that they were going to smell chocolate and therefore may have viewed the chocolate pictures in a different way than the controls did right from the start.

Our findings that the brain might have precursory characteristics are in line with results from studies with alcoholics (Grüsser et al., 2004) and treatment-seeking cocainedependent participants (Kosten et al., 2006). In these participants, brain activation in reward regions in response to cues of the respective drug of abuse proved to be a better predictor of relapse than subjective craving reports. Even when participants were asked to rate their implementation intentions (instead of craving), brain activation was a better predictor of subsequent behaviour than were subjective reports, as shown in studies with smokers (Falk et al., 2011) and in people who were exposed to persuasive messages regarding the value of regular sunscreen use (Falk et al., 2010). This shortcoming of subjective reports with regard to predictive validity may be due to interoceptive awareness being limited in some of the individuals (Craig, 2004; Herbert \& Pollatos, 2012).

In the present study, we did not find the hypothesised decrease in subjective craving with repeated exposure to chocolate. This may be due to the frequent interruption of the exposures by the brain scans. This interruption may have prevented the extinction of subjective craving in the chocolate exposure group. In addition, the duration of the final prolonged exposure was probably not long enough. However, a slight decrease in craving in the chocolate exposure group was found at the very end of the final prolonged exposure and this might have continued with further exposure. Apart from these considerations, the use of a different food cue than chocolate might have yielded different results, because chocolate is a generally highly preferred food. It has been found to resist extinction in a previous study that studied the effects of context change on acquisition and extinction of conditioned chocolate craving (Van Gucht, Vansteenwegen, Beckers, $\&$ Van den Bergh, 2008). In addition, there was a marginally significant group difference of BMI in our sample, which may have influenced the results (see Table 1). However, the mean BMI of each group was well within the healthy-weight range and only one participant was slightly overweight. Finally, no menstrual cycle data was collected, which may have introduced some additional noise, since the neural response to food cues fluctuates throughout the menstrual cycle (Dreher et al., 2007). 
Taken together, the results of this study indicate that the changes in brain reward activitation during CERP are linked to the changes in craving, at least for a short exposure. This is substantiated by an increase of these measures in the experimental group, compared with constant values for the control group. Therefore, a short cue exposure can be considered effective in increasing both craving and brain reward activation. Regarding the long exposure, the expected extinction of subjective craving did not occur, although a decrease of craving was found at the very end of the prolonged exposure, which might have continued if the exposure would have been even longer. At the same time, brain reward activation did decrease at the end of the session. Therefore, the decreased brain reward activation may be considered a precursor of a decrease in craving. To test this possibility, brain activation and subjective measurements before and after a longer CERP would be necessary. In any case, these results show that CERP is an effective intervention for the reduction of brain reward activation as a form of food cue reactivity. This treatment should be further investigated as a possible therapy for overweight or obese people to cope with their excessive food craving.

\section{ACKnowledgements}

We would like to thank Ing. Charlie Bonnemayer of Maastricht University for his advice on the programmation of the stimulus protocol in E-Prime, as well as Dr. Armin Heinecke of Brain Innovation B.V. for his support during the data analysis. This work was supported by the Netherlands Organisation for Scientific Research (NWO) (grant number 400.06.148 to Prof. Dr. Anita Jansen). 


\section{Chapter 5}

\section{NeURAL PREDictors OF CHOCOLATE INTAKe FOLLOWING CHOCOLATE EXPOSURE}

Frankort, A., Roefs, A., Siep, N., Roebroeck, A., Havermans, R., \& Jansen, A. (n.d.). Neural predictors of chocolate intake following chocolate exposure. Manuscript in revision at NeuroImage 


\begin{abstract}
Previous studies have shown that one's brain response to high-calorie food cues can predict long-term weight gain or weight loss. The neural correlates that predict food intake in the short term have however hardly been investigated. This study examined which brain regions predict chocolate intake after participants had been either exposed to chocolate or to control stimuli during approximately one hour. Further we investigated whether the variance in chocolate intake could be better explained by activated brain regions than by self-reported craving. In total, five brain regions correlated with subsequent chocolate intake. Two reward regions (the right caudate and the left frontopolar cortex) correlated positively with intake in the exposure group. Two regions associated with cognitive control (the left dorsolateral and left mid-dorsolateral PFC) correlated negatively with intake in the control group. When the regression analysis was conducted with the exposure and the control group together, an additional region (the right anterior PFC) correlated positively with chocolate intake. In all analyses, the intake variance explained by neural correlates was above and beyond the variance explained by self-reported craving. These results are in line with neuroimaging research showing that brain responses are a better predictor of subsequent intake than self-reported craving. Therefore, our findings might provide for a missing link by associating brain activation, previously shown to predict weight change, with short-term intake.
\end{abstract}




\section{INTRODUCTION}

Self-reports of attitudes and intentions rely on introspection, and it is well known that people do not have introspective access to all causes that underlie their behaviour (Nisbett \& Wilson, 1977) and that these self-reports can be distorted by socially desirable answering tendencies (Schwarz \& Oyserman, 2001). Recently, neuroimaging research showed that brain responses have added value in the prediction of behaviour. In participants who were exposed to persuasive messages regarding the value of regular sunscreen use or the benefits of quitting smoking, for example, brain activation was a better predictor of subsequent behaviour than were self-reports of intentions (Falk, Berkman, Mann, Harrison, \& Lieberman, 2010; Falk, Berkman, Whalen, \& Lieberman, 2011). Likewise, in addicted participants, the brain response to pictures or videos of cues of the particular drug was a better predictor for relapse than were subjective craving reports (Grüsser et al., 2004; Kosten et al., 2006). Studies investigating the neural responsivity to high-calorie food cues also found that this activation in brain reward regions was correlated with weight gain after one year (Stice, Yokum, Bohon, Marti, \& Smolen, 2010; Yokum, Ng, \& Stice, 2011a) or with less success in losing weight in a subsequent weight-loss program (Murdaugh, Cox, Cook, \& Weller, 2012).

Although many studies in the last few years have investigated the differences between healthy-weight and overweight people with regard to neural correlates of food reward (e.g., Batterink, Yokum, \& Stice, 2010; Frankort et al., 2012; Rothemund et al., 2007; Stoeckel et al., 2008), the neural correlates in response to visual food cues that predict subsequent food intake in the short term has, to our knowledge, only been studied once (Lawrence, Hinton, Parkinson, \& Lawrence, 2012). This study found that snack consumption after the scanning session was predicted by activation of the nucleus accumbens in response to food cues. Prediction of the amount of food intake is interesting because weight gain is the result of a sustained positive energy balance (Swinburn et al., 2009). The goal of this study was to examine whether neural correlates of food reward and inhibition could predict food intake during an alleged taste-test. The predictive validity of these neural correlates was studied under two conditions: during a session of exposure to the smell of chocolate without eating and during a control exposure (no food). Neural responses to chocolate pictures versus neutral pictures were measured using functional magnetic resonance imaging (fMRI).

Prolonged exposure is a well-known treatment strategy in the clinical practice of mental health care. During food cue exposure with response prevention (CERP), one is exposed to food cues, like the smell or taste of foods, or contexts, like eating places, that predict food intake, while at the same time eating is not allowed (response prevention). The exposure aims to disrupt the conditioned link between the food cue (sight, smell or context of food intake) and one's learned response to this cue (eating). Actually, during repeated prolonged effective exposure it is learned that the food cue or context does not 
predict intake anymore (see e.g., Jansen, Havermans, \& Nederkoorn, 2011). During the exposure, levels of self-reported craving first increase during prolonged exposure, peak and then slowly decrease to the end. After several sessions the experienced craving does not go up anymore and almost fully extinguishes (see, e.g., Van Gucht et al., 2008; Jansen, Broekmate, \& Heymans, 1992; Jansen, Van den Hout, De Loof, Zandbergen, \& Griez, 1989; Martinez-Mallén et al., 2007; Toro et al., 2003). In our lab, we addressed the effect of prolonged exposure to the smell of chocolate, without eating, on self-reported cravings and brain responses to neutral and chocolate pictures (Frankort et al., 2013). It was found that self-reported chocolate craving in a group that was exposed to chocolate increased over a one-hour session, reaching a peak just before the end of the session, followed by a tendency towards a decrease. Contrary to expectations, chocolate cravings did not extinguish within the hour. However, activation in brain reward regions in response to chocolate versus neutral pictures rose from the start to the middle of the session. At the end of the session the brain reward activation returned to control levels again, indicating that craving on a neural level did extinguish. Therefore, brain reward activation was considered to be a precursor of self-reported craving (Frankort et al., 2013).

The current study follows up on these results and focuses specifically on chocolate intake during a bogus taste-test that was performed after the fMRI scanning session. We expected that chocolate exposure would lead to reduced intake as compared to the neutral control exposure, because a prolonged exposure usually leads to a decrease in craving. Further it was expected that brain activation in response to chocolate versus neutral pictures in regions associated with food reward would show predictive validity with regard to subsequent intake above and beyond the variance explained by self-reported craving. Brain regions commonly accepted to be involved in food reward are the amygdala, striatum, hippocampus, insula, ventral tegmental area and substantia nigra, as well as the anterior cingulate, posterior fusiform, orbitofrontal and prefrontal cortices (for reviews see: Berridge, Ho, Richard, \& DiFeliceantonio, 2010; Berthoud, Lenard, \& Shin, 2011; Carnell, Gibson, Benson, Ochner, \& Geliebter, 2012; Kringelbach, 2009; Van der Laan, De Ridder, Viergever, \& Smeets, 2011; Rolls, 2010; Small, 2009). Regions that appear to be involved in the inhibition of food reward, in the sense that less activation in a delay discounting task or a lower grey matter volume of these regions predicted more long-term weight gain in women, are subdivisions of the prefrontal cortex (PFC) like ventrolateral, dorsolateral and medial PFC, as well as posterior parietal cortex (Kishinevsky et al., 2012; Stoeckel, Murdaugh, Cox, Cook, \& Weller, 2012; Yokum, Ng, \& Stice, 2011b). Therefore, more specifically, we expect activation in these regions to explain variance in chocolate intake (as measured directly after the scanning session), on top of what is predicted by self-reported craving. To test whether chocolate exposure increases the predictive validity of brain reward activation on intake, the regression of brain activation on chocolate intake was not only performed with participants of the whole sample but also for each group separately. 


\section{Method}

Part of these data have been published in a previous paper (Frankort et al., 2013), which focused on the course of brain activation during the cue exposure session. The current paper investigates the prediction of short-term chocolate intake after the session by brain activation.

\section{Participants}

Thirty-four female undergraduate students of Caucasian ethnicity from Maastricht University participated (17 per group) with full informed consent. They were right-handed, not currently on a weight-loss diet and had a low score (total score < 14) on the Restraint Scale (Herman \& Polivy, 1980). Exclusion criteria were a self-reported mental disorder history or family history of eating disorders. Both groups had similar age and scores of hunger, restraint and trait chocolate craving, but body mass index (BMI) tended to differ between the groups (see Table 1 for participant characteristics). Seven additional participants were excluded from analyses due to scanner problems or excessive body movement. Participation was remunerated with $€ 30$ or course credits. The local ethical committee approved the study.

\section{Assessment}

Self-reported chocolate craving. During the session, self-reported chocolate craving was measured repeatedly with a visual analogue scale (VAS) item asking "how much do you crave chocolate at this moment", ranging from 0 (not at all) to 100 (very much).

Trait chocolate craving. To assess the trait craving for chocolate and the degree of compulsive behaviour elicited by chocolate, the craving subscale of the Attitudes to Chocolate Questionnaire (Benton, Greenfield, \& Morgan, 1998) was used. All items addressed preoccupation with chocolate, with the scale for each item ranging from 0 (this does not apply to me at all) to 100 (this very much applies to me). The subscale is considered valid and reliable (Cramer \& Hartleib, 2001; Müller, Dettmer, \& Macht, 2008). The total score was computed by averaging the ten VAS items ( 0 to 100$)$.

Hunger. A VAS item asking "How hungry do you feel at this moment?", ranging from 0 (not hungry at all) to 100 (very hungry) was used to assess hunger.

Restraint. The participant's intention to restrain her food intake, her concern about body weight and weight fluctuations were measured with the Restraint Scale (Herman \& Polivy, 1980), with a minimum total score of 0 and a maximum of 35. A higher score reflects a higher level of restraint. The scale is sufficiently valid and reliable (Scagliusi et al., 2005; Van Strien, Herman, Engels, Larsen, \& Van Leeuwe, 2007; Williamson et al., 2007). 
Bogus chocolate taste test. In a bogus chocolate taste test, participants were asked to taste four kinds of chocolate buds (white, milk, dark, extra dark; Barry Callebaut Belgium N.V., Wieze, Belgium). The separate bowls in which they were presented were weighed prior to the test and contained approximately $250 \mathrm{~g}$ of chocolate each. Participants were instructed that they could taste as many chocolate buds as needed to complete the taste ratings, and that they were free to consume chocolate buds after they had finished. The chocolate's smell, taste, intensity, appearance, taste persistence and sweetness were rated with six VAS items (0 to 100). The overall intake of chocolate was measured by adding up the consumed weights of the four different chocolate types.

\section{Experimental Design}

The experimental design was a mixed design with an exposure group (exposure to chocolate) versus a control group (exposure to a control stimulus), with repeated measures of both self-reported chocolate craving and the brain response to chocolate pictures versus neutral control pictures. Chocolate intake was measured after the session. For the current study, only brain responses in the last run (run 7) were analysed because they were closest in time to subsequent intake.

Table 1. Characteristics and self-report data of participants

\begin{tabular}{|c|c|c|c|c|c|c|}
\hline & \multicolumn{2}{|c|}{ Exposure group } & \multicolumn{2}{|c|}{ Control group } & \multirow[b]{2}{*}{$t(32)$} & \multirow[b]{2}{*}{$p$} \\
\hline & $M$ & $S D$ & $M$ & $S D$ & & \\
\hline Age (yrs) & 19.8 & 1.4 & 20.2 & 1.5 & .89 & .38 \\
\hline BMI & 22.8 & 1.7 & 21.6 & 1.8 & 1.94 & .06 \\
\hline Time between lunch and scan (min) & 86.5 & 14.4 & 82.6 & 20.9 & .62 & .54 \\
\hline Hunger $^{\mathrm{a}}$ & 18.6 & 14.5 & 14.9 & 14.1 & .74 & .46 \\
\hline Restraint $^{\mathrm{b}}$ & 10.6 & 2.6 & 10.8 & 4.7 & .18 & .86 \\
\hline Trait chocolate craving ${ }^{c}$ & 38.3 & 19.1 & 35.0 & 13.2 & 0.58 & .56 \\
\hline Overall chocolate intake (g) & 19.6 & 15.8 & 24.7 & 16.4 & .92 & .36 \\
\hline
\end{tabular}

Note. Reprinted with permission from Frankort et al. (c) 2013 Cerebral Cortex.

Abbreviations. $\mathrm{BMI}=$ Body Mass Index.

aScored on 100 mm VAS, ranging from 0 (not hungry at all) to 100 (very hungry).

${ }^{\mathrm{b} S}$ cored on the Restraint Scale (Herman \& Polivy, 1980), with a minimum total score of 0 (no restraint) and a maximum of 35 (high restraint).

'Scored on the craving subscale of the Attitudes to Chocolate Questionnaire (Benton et al., 1998), with a minimum total score of 0 (no trait chocolate craving) and a maximum of 100 (high trait chocolate craving). 


\section{Stimuli}

56 pictures of (products containing) chocolate and 56 neutral pictures of office supplies and utensils not related to food were used as stimuli. Only chocolate pictures without festive associations (such as Valentine, Easter and birthday) were used. Pictures were predominantly purchased online (www.istockphoto.com). Stimuli covered a visual angle of approximately $12^{\circ}$ and were presented in the centre of the screen as pop-out figures on a light grey background. Each picture was presented four times in the course of the scanning session.

\section{Stimulation Protocol}

A blocked design with a block duration of $14.8 \mathrm{~s}^{1}$ was used to present the stimuli (EPrime version 2.0.8.90 (PST, 1996)). All blocks consisted of eight pictures with a duration of $1850 \mathrm{~ms}$ each, which were chosen randomly without replacement. Half of the stimulus blocks were followed by a question block of $3 \mathrm{~s}$ duration with a yes/no question about the depicted picture having been presented in the preceding stimulus block. This was done to maintain the attention of the participant, who responded with a button press. A black fixation cross on a light grey background preceded all blocks, with a duration of either $12 \mathrm{~s}$ (before stimulus blocks) or $6 \mathrm{~s}$ (before question blocks). After the last block an additional fixation cross was shown for $12 \mathrm{~s}$.

One run consisted of four chocolate blocks, four neutral blocks, and four question blocks. The order of stimulus blocks was randomised for each of the seven runs, with the constraint that a maximum of two stimulus blocks of the same category would occur subsequently. Question blocks were then inserted randomly, with the constraint that twice they followed a chocolate block and twice a neutral block. This resulted in seven fixed run orders that lasted on average for $270 \mathrm{~s}^{2}$. For each participant, the sequence of these seven runs was randomised.

\section{Session Protocol}

The session of 65 min duration started with the first functional run, after which the first exposure took place. Functional runs and exposures were alternated during the scanning session. For exposure, the participant remained in the scanner and was handed a piece of chocolate (in the exposure group) or a pencil (in the control group), with the instruction to hold it under her nose and smell it. The piece of chocolate (approximately $4 \mathrm{~cm} \times 2 \mathrm{~cm} \mathrm{x} 1 \mathrm{~cm}$ ) was partly wrapped in an odourless tissue to prevent it from melting. Participants in the chocolate exposure group were instructed to try to crave the chocolate as much as possible, by imaging eating it and feeling it melting in their mouth. 
The objects were taken back at the end of each exposure. Therefore, there was no exposure to real chocolate or to the pencil during functional runs. Habituation to the smell of the cue was counteracted by instructing the participant to ask for a different one of in total eight brands of milk and dark chocolate (in the exposure group) or a different one of eight pencils (in the control group). In practice, this led to a replacement of the cue at least every few minutes. Every functional run started and ended with a VAS item about the participant's chocolate craving, which could be scored using a joystick on her abdomen. Chocolate craving was additionally assessed every five minutes in the final prolonged exposure cycle, which lasted $20 \mathrm{~min}$. The other exposures were rather short and amounted to $1,1,2,3$ and 3 min respectively. The anatomical measurement was done after the last functional run.

\section{Procedure}

The scanning sessions took place in the afternoon and consisted of seven functional runs and one anatomical measurement. On the scanning day, participants were asked to refrain from eating chocolate products and from drinking coffee or tea (Koppelstaetter et al., 2010). They had to consume a regular lunch 1 to 1.5 hours before the start of the scanning session. Upon arrival, the participant's hunger, restraint, trait craving for chocolate, weight and height were measured. After the participant gave informed consent the scanning session started. After the scanning session, the participant did the bogus chocolate taste test and filled in an exit form in an adjacent room. Then she was thanked and compensated for participation. Debriefing was done by e-mail at the end of the study.

\section{fMRI Data Acquisition}

A 3T Siemens Magnetom Allegra head-only scanner was used for image acquisition. Functional T2*-weighted images ( 50 slices, $\mathrm{TR}=3000 \mathrm{~ms}$ ) were acquired using gradientecho planar imaging (EPI) parameters, optimised for reducing susceptibility and distortion artefacts in the OFC (Deichmann, Gottfried, Hutton, \& Turner, 2003; Weiskopf, Hutton, Josephs, Turner, \& Deichmann, 2007): TE $=25 \mathrm{~ms}$, oblique axial slices acquired in interleaved order with a negative (i.e. backward) tilt angle of $30^{\circ}$, voxel size $=2 \mathrm{~mm} \times 2$ $\mathrm{mm} \times 2.5 \mathrm{~mm}$, field of view $(\mathrm{FoV})=256 \mathrm{~mm} \times 192 \mathrm{~mm}$, imaging bandwidth $=2790 \mathrm{~Hz} /$ pixel, echo spacing $=0.42 \mathrm{~ms}$, flip angle $=90^{\circ}$. Each run contained 88 volumes. An optimised anatomical T1-weighted magnetization-prepared rapid gradient-echo sequence (Deichmann, Good, Josephs, Ashburner, \& Turner, 2000; Mugler \& Brookeman, 1990) was used after the last run, with the following settings: $\mathrm{TR}=2250 \mathrm{~ms}$, TE $=2.6 \mathrm{~ms}$, flip angle $=9^{\circ}$, voxel size $=1 \mathrm{~mm} \times 1 \mathrm{~mm} \times 1 \mathrm{~mm}$, echo spacing $=6.9 \mathrm{~ms}$. 


\section{Data Preprocessing}

Preprocessing and first-level analyses were conducted with BrainVoyager QX version 2.4.1.2052 (BrainInnovation, 2001). The first two volumes of each functional run were excluded due to T1 saturation effects. Preprocessing involved slice scan time correction with cubic spline interpolation, low-frequency noise removal with high-pass temporal filtering ( $0.0075 \mathrm{~Hz}$ cut-off), and three-dimensional motion correction, using trilinear interpolation for alignment and sinc interpolation for final resampling. Preprocessed data were then aligned to the anatomical scan, resulting in co-registered three-dimensional space data with a voxel resolution of $2 \mathrm{~mm} \times 2 \mathrm{~mm} \times 2 \mathrm{~mm}$ over the course of time for each run. These data were subsequently spatially normalised using Talairach transformation procedures (Talairach \& Tournoux, 1988) and spatially smoothed with a $6 \mathrm{~mm}$ full-width-at-half-maximum isotropic Gaussian Kernel. For group-level analyses, anatomical Talairach-standardised participant data sets were averaged. On this basis a whole-brain mask was generated.

\section{Data Analysis}

Regarding overall chocolate intake, group differences were analysed with a $t$ test. This was followed by the calculation of Pearson correlations of chocolate intake with self-reported chocolate craving, restraint scale scores, trait chocolate craving scores, hunger and time passed between lunch and the start of the scanning session. For brain responses boxcar predictors were set for chocolate, neutral, and question blocks of the last run (run 7). Question block predictors were considered as confounds. A standard hemodynamic response function (Friston et al., 1998) was used to convolve all predictors. The design matrix of the general linear model was generated after adding $z$-transformed motion correction parameters. This modelling approach considers the response to fixation times between blocks as a baseline.

In three separate whole-brain regression analyses, brain responses in the last run (run 7) to chocolate pictures versus neutral pictures were correlated with the total chocolate intake for the whole sample (first analysis) as well as separately for the exposure group (second analysis) and the control group (third analysis). Only voxels with a $p$ value below 0.05 were considered significant, corrected for multiple comparisons at the cluster level by using a Monte Carlo simulation based approach, with 1000 iterations and a voxel size of $2 \mathrm{~mm} \times 2 \mathrm{~mm} \times 2 \mathrm{~mm}$ (Forman et al., 1995; Goebel, Esposito, \& Formisano, 2006). In total 191443 voxels were contained in the Talairach-standardised whole-brain mask. As a result of these calculations, the minimal cluster size of functional regions of interest (fROIs) was set to $328 \mathrm{~mm}^{3}$ (first analysis, whole sample), $336 \mathrm{~mm}^{3}$ (second analysis, exposure group only), and $352 \mathrm{~mm}^{3}$ (third analysis, control group only). The Talairach Client (www.talairach.org) (Lancaster et al., 2000) was used to indicate the anatomical 
localization of the fROIs. To obtain activation characteristics of these fROIs, average $\beta$ values were extracted for each fROI, run, picture category and participant and were entered into SPSS version 19 for visualization purposes.

\section{Regression Model}

To test the added value of neural activation in run 7 to self-reported chocolate craving for the prediction of chocolate intake, three regression models were tested: one for the whole sample, one for the exposure group, and one for the control group. In each model, in step 1 self-reported craving was entered in the model, and in step 2 neural activation that correlated with chocolate intake was added. The self-reported chocolate craving was the optimal score of self-reported chocolate craving, as determined by the highest correlation between self-reported chocolate craving and intake (see Table 2). The neural activation was centered before it was entered into the model and was based on the response to chocolate pictures versus neutral pictures that correlated with chocolate intake, in clusters larger than the threshold in each regression analysis (whole sample, exposure group and control group).

\section{RESULTS}

\section{Chocolate intake and self-reported craving}

Overall chocolate intake did not differ between groups (see Table 1), although it had been expected that chocolate CERP would lead to reduced intake as compared to the neutral control exposure. Chocolate intake was not correlated with restraint scale scores, trait chocolate craving scores, hunger, or time passed since lunch was eaten, in either the exposure or the control group, or the whole sample (see Table 2). No significant correlations were found between self-reported chocolate craving and subsequent intake in the whole sample or just the control group. However, within the exposure group, chocolate craving showed significant correlations with overall chocolate intake. The craving score that correlated maximally with intake was entered into the regression models.

\section{Neural predictors of chocolate intake}

The whole-brain regression analysis (for the whole sample) yielded one fROI larger than the minimal cluster size, in which the activation (response to chocolate pictures vs. neutral pictures) of all individual voxels was positively correlated with intake. This fROI was located in the right anterior PFC (cluster A in Figure 1 and Table 3). The second analysis, performed with only the exposure group, resulted in two fROIs (clusters B and $\mathrm{C}$ in Figure 1 and Table 3 ) with a positive correlation of activation and subsequent chocolate 
Table 2. Correlations of overall chocolate intake with participant characteristics and measurements

\begin{tabular}{|c|c|c|c|c|}
\hline & & $\begin{array}{l}\text { Whole sample } \\
(\mathrm{n}=34)\end{array}$ & $\begin{array}{l}\text { Exposure group } \\
(n=17)\end{array}$ & $\begin{array}{l}\text { Control group } \\
(n=17)\end{array}$ \\
\hline \multicolumn{2}{|l|}{ Hunger $^{a}$} & -.17 & -.10 & -.20 \\
\hline \multicolumn{2}{|c|}{ Time between lunch and scan session } & .10 & -.02 & .22 \\
\hline \multicolumn{2}{|l|}{ Restraint $^{\mathrm{b}}$} & .24 & .23 & .25 \\
\hline \multicolumn{2}{|c|}{ Trait chocolate craving ${ }^{c}$} & .17 & .18 & .21 \\
\hline \multicolumn{5}{|c|}{ Self-reported chocolate craving ${ }^{d}$} \\
\hline \multirow{2}{*}{ Run 1} & Before & .05 & .01 & .05 \\
\hline & After & .11 & .24 & .04 \\
\hline \multirow{2}{*}{ Run 2} & Before & .09 & .26 & .01 \\
\hline & After & .14 & .25 & .09 \\
\hline \multirow{2}{*}{ Run 3} & Before & .10 & .36 & .01 \\
\hline & After & $.19^{\ddagger}$ & .32 & .14 \\
\hline \multirow{2}{*}{ Run 4} & Before & .16 & $.53^{*}$ & -.03 \\
\hline & After & .08 & .35 & -.05 \\
\hline \multirow{2}{*}{ Run 5} & Before & .07 & .46 & -.10 \\
\hline & After & .07 & .36 & -.08 \\
\hline \multirow{2}{*}{ Run 6} & Before & .07 & $.52^{\star}$ & -.15 \\
\hline & After & .08 & .46 & -.08 \\
\hline \multirow{3}{*}{$\begin{array}{l}\text { During prolonged } \\
\text { exposure }(20 \\
\text { min) }\end{array}$} & At $5 \mathrm{~min}$ & .12 & $.61^{* * \neq}$ & -.14 \\
\hline & At $10 \mathrm{~min}$ & .06 & $.53^{*}$ & -.16 \\
\hline & At $15 \mathrm{~min}$ & .10 & $.57^{*}$ & -.12 \\
\hline \multirow{2}{*}{ Run 7} & Before & .06 & $.50^{*}$ & $-.20^{\ddagger}$ \\
\hline & After & .09 & .42 & -.10 \\
\hline
\end{tabular}

Note. Significance indications: ${ }^{*} p<.05 ;{ }^{* *} p<.01$ (uncorrected).

a-c See notes of Table 1.

${ }^{\mathrm{d} S}$ cored on $100 \mathrm{~mm}$ VAS, ranging from 0 (not craving chocolate at all) to 100 (craving chocolate very much).

${ }^{*}$ Highest correlation coefficient of the respective analysis (see column head) between self-reported chocolate craving and intake.

intake. These were located in the right caudate and the left lateral frontopolar cortex. The third regression analysis with only the control group produced two fROIs (clusters D and $\mathrm{E}$ in Figure 1 and Table 3) with a negative correlation between activation and intake, in the left dorsolateral and the left mid-dorsolateral PFC surrounding the inferior frontal sulcus. Independent samples $t$ tests showed that within each cluster there was no group difference regarding the average activation per group, all $t \mathrm{~s}<.67$, all $p \mathrm{~s}>.51$. 


\section{Regression model with self-reported craving and neural corre- lates of intake}

Step 1 of the regression models showed that the regression of self-reported craving on intake was not significant in the whole sample and in the control group. In the exposure group, the model showed that intake increased with increasing optimal self-reported craving (Table 4), which accounted for more than one third of explained variance. After adding neural activation to the model in step 2, all three regression analyses (one with the whole sample, one with only the exposure group and one with only the control group) were significant. Variance explained by neural activation was above and beyond the variance explained by self-reported craving alone in all three regression analyses. Significant contributions to explained variance were made by the right anterior PFC (cluster A in the analysis with the whole sample), the right caudate (cluster B in the exposure group analysis) and, with a negative sign, the left mid-dorsolateral PFC (cluster $\mathrm{E}$ in the control group analysis).

Table 3. fROIs: Clusters larger than the calculated minimal cluster size of the respective analysis (whole sample, exposure group or control group). In these clusters, all voxels significantly correlated with overall chocolate intake in terms of their activation in response to chocolate pictures versus neutral pictures

\begin{tabular}{|c|c|c|c|c|c|c|c|}
\hline \multirow{2}{*}{ fROI } & \multirow{2}{*}{ Anatomical label } & \multirow{2}{*}{$\begin{array}{c}\text { Sign of } \\
\text { correla- } \\
\text { tion }\end{array}$} & \multirow{2}{*}{$\begin{array}{c}\text { Esti- } \\
\text { mated } \\
\mathrm{BA}^{\mathrm{b}}\end{array}$} & \multirow{2}{*}{ size $\left(\mathrm{mm}^{3}\right)$} & \multicolumn{3}{|c|}{ Peak voxel values ${ }^{\mathrm{a}}$} \\
\hline & & & & & $\mathrm{x}$ & $\mathrm{y}$ & $\mathrm{z}$ \\
\hline \multicolumn{8}{|c|}{ Whole sample $(n=34)$} \\
\hline $\mathrm{A}$ & anterior PFC (MFG) $\mathrm{R}^{\star * *}$ & + & 10,9 & 412 & 35 & 38 & 23 \\
\hline \multicolumn{8}{|c|}{ Exposure group $(n=17)$} \\
\hline B & $\begin{array}{l}\text { caudate body, } \\
\text { claustrum } \mathrm{R}^{* *}\end{array}$ & + & & 501 & 19 & 11 & 23 \\
\hline $\mathrm{C}$ & $\begin{array}{l}\text { lateral frontopolar cortex } \\
(\mathrm{MFG} / \mathrm{SFG}) \mathrm{L}\end{array}$ & + & $10,9,46$ & 412 & -40 & 53 & 23 \\
\hline \multicolumn{8}{|c|}{ Control group $(n=17)$} \\
\hline $\mathrm{D}$ & dlPFC (MFG) L & - & 6 & 379 & -46 & 7 & 51 \\
\hline $\mathrm{E}$ & $\begin{array}{l}\text { mid-dlPFC surrounding the } \\
\text { IFS (MFG/IFG) L } L^{*}\end{array}$ & - & 46,45 & 433 & -55 & 25 & 19 \\
\hline
\end{tabular}

Note. Significance indications for predictors in the regression model: ${ }^{*} p<.05 ;{ }^{* *} p<.01 ;{ }^{* * *} p<.001$ (uncorrected). Abbreviations. $\mathrm{BA}=$ Brodmann Area; $\mathrm{dlPFC}=$ dorsolateral PFC; IFG = inferior frontal gyrus; IFS = inferior frontal sulcus; $\mathrm{L}=$ left hemisphere; $\mathrm{MFG}=$ middle frontal gyrus; $\mathrm{R}=$ right hemisphere; $\mathrm{SFG}$ = superior frontal gyrus. ${ }^{a}$ Voxel coordinates are reported in Talairach space.

'Identified with the "nearest grey matter" option in the Talairach Client (www.talairach.org) (Lancaster et al., 2000). 

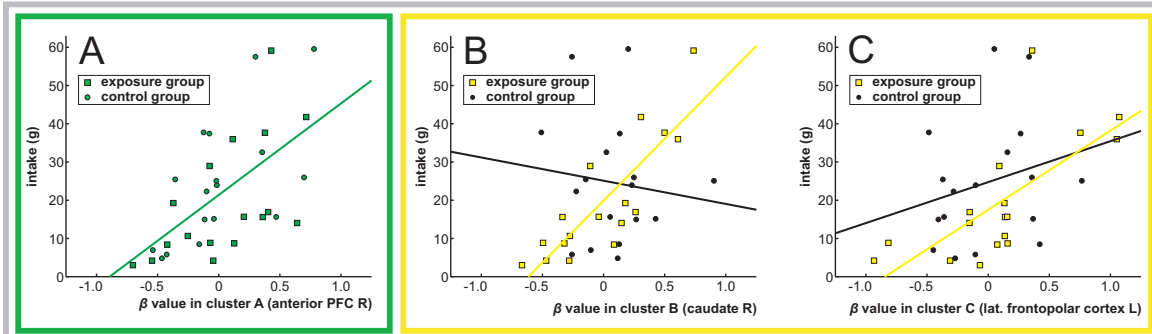

analysis with group as a whole $(n=34)$

analys:5 woth exposure group (n
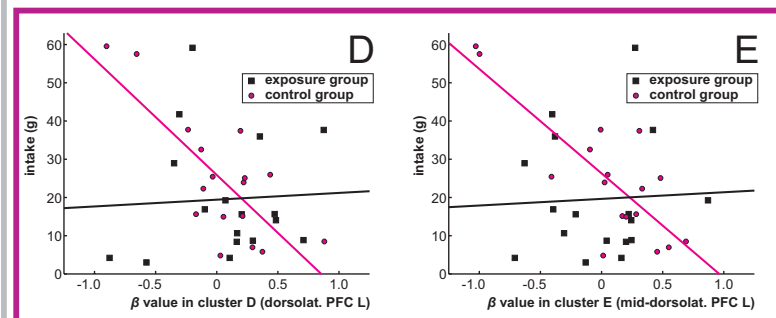

analysis with control group

$\mathrm{E}$ $(n=17)$

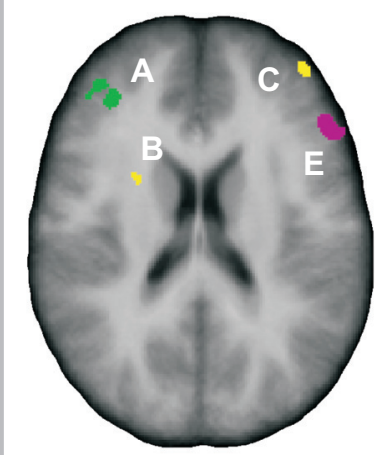

$z=20$

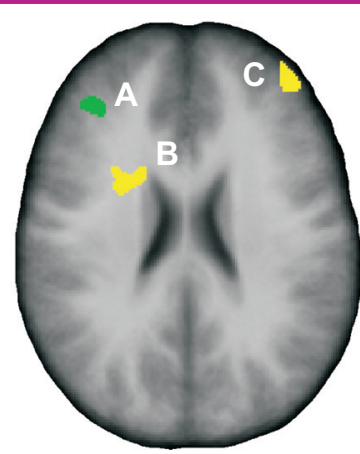

$z=24$

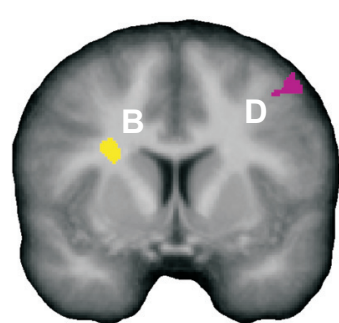

$$
y=5
$$

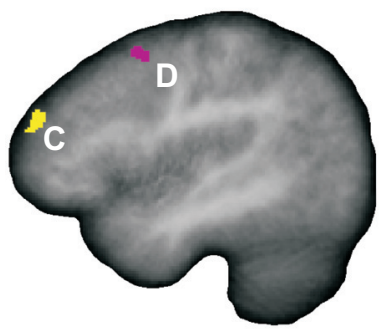

$x=-43$

Figure 1. Upper part: Scatter plots of clusters resulting from three regression analyses of average $\beta$ values, as extracted from neural activation of all voxels in the corresponding cluster in response to chocolate pictures versus neutral pictures, on overall chocolate intake. Lower part: Locations of clusters that significantly correlate with overall chocolate intake after the scanning session. One cluster (A, green) resulted from the regression analysis of the whole sample; the regression analysis of the exposure group (B and C, yellow) and the control group ( $\mathrm{D}$ and E, blue) yielded two clusters each. Only clusters $\mathrm{A}, \mathrm{B}$ and $\mathrm{E}$ were significant predictors of intake in the regression model. 


\section{Discussion}

Building on a previous study (Frankort et al., 2013), the current study investigated the self-reported and neural predictors of chocolate intake after having been exposed during approximately one hour to chocolate (exposure group) or to a control stimulus (control group). Three regression models were tested, that is, for each group separately and for the whole sample. The regression models tested if there are neural correlates in brain regions associated with reward that predict intake, and if the variance explained by these neural correlates is above and beyond the variance explained by self-reported craving. For the whole sample, self-reported chocolate craving did not correlate at any point in time with subsequent intake, which was unexpected. Interestingly, activation in the right anterior PFC was positively correlated with intake and significantly contributed to the explained variance of intake. Although the function of this region is not very specific as it is active during multiple and diverse cognitive tasks (Ramnani \& Owen, 2004), a systematic review of brain lesions in eating disorders (Uher \& Treasure, 2005) found a consistent association of right frontal lobe lesions with weight loss. The same region's response to appetizing food versus water was found to correlate positively with BMI (Stice et al., 2010). Moreover, the activation of this part of the PFC has been associated with the intake of alcohol in abstinent alcoholics who subsequently relapsed (Grüsser et al., 2004). Therefore, the predictive validity of this region's activation on intake is in line with existing literature.

The control group showed no correlation between self-reported craving during the session and chocolate intake after the session. On a neural level, two brain regions in the left dorsolateral and mid-dorsolateral PFC correlated with intake, with the latter contributing significantly to explained variance of intake. Correlation with chocolate intake was negative in both regions, which is not surprising given the fact that both have been associated with cognitive control, self-control and inhibition (Coutlee \& Huettel, 2012; Figner et al., 2010; Petrides, 2005). More specifically, an increase in regional cerebral blood flow in the vicinity of these regions was associated with a decrease in reward value (Small, Zatorre, Dagher, Evans, \& Jones-Gotman, 2001) and in a prospective study, activation in both regions in response to food versus control stimuli was predictive of subsequent weight loss after 12 weeks (Murdaugh et al., 2012). Moreover, a voxel-based morphometry study showed that a reduction of grey matter in almost exactly the same region in the left dorsolateral PFC was predictive of an increase in BMI after one year (Yokum et al., 2011b). The negative correlations in our study with control group intake confirm that these regions are implicated in the inhibition of food reward. Slightly surprising was the fact that only brain inhibitory regions had an influence on intake in the control group (instead of brain reward regions). It appeared that, at the end of the session, inhibitory activation in response to chocolate versus neutral pictures had a larger influence on subsequent intake than reward activation in the control group, although the average activation in the mentioned fROIs did not differ from the average activation in the exposure group. 
The exposure group did show a correlation of self-reported craving with chocolate intake, for several time points in the scanning session. The optimal craving rating accounted for $37 \%$ of the explained variance of intake. Intake also correlated with neural activation in the right caudate and the left lateral frontopolar cortex, with a significant contribution of the caudate activation to explained variance of intake, above and beyond the amount explained by craving. The more active these regions were, the more the chocolate-exposed participants ate after the session. Although the frontopolar cortex has been found to exercise control over highly abstract plans, goals, and response strategies (Coutlee \& Huettel, 2012), regions in left lateral frontopolar cortex close to that identified here have also been implicated in reward and craving, like monetary reward (Pochon et al., 2002) and expectation of or craving for cocaine (Goldstein \& Volkow, 2011; Gorelick et al., 2005), but it also seems to be active during many complex cognitive tasks (Ramnani \& Owen, 2004). Activation of the other cluster in this exposure group analysis, the caudate, has been correlated with the reward value of chocolate (Small et al., 2001) and with meal pleasantness in general (Small, Jones-Gotman, \& Dagher, 2003). In obese participants, the caudate nucleus has been found to be more activated than in healthy-weight controls in response to pictures of food versus neutral pictures (Rothemund et al., 2007; Stoeckel et al., 2008). The increased caudate activation predicting increased chocolate intake after chocolate exposure fits with these findings. It is surprising, though, that the correlation between activation in the caudate and intake was restricted to the exposure group, while average activation levels were comparable in both groups. Similarly, the correlation between self-reported craving and intake was restricted to the exposure group as well. Apparently the chocolate exposure manipulation did influence the way in which the chocolate stimuli were perceived and also influenced self-reported craving in a way that it predicted intake. It could be that the exposure group, because of the chocolate exposure and the instruction to imagine eating the chocolate, was more conscious about their chocolate craving, which may have resulted in a correlation between self-reported craving and subsequent intake.

As these results show, the CERP manipulation did have an influence on the predictive validity of neural correlates of chocolate craving on intake. However, the CERP manipulation was not as effective as it was expected to be, because overall chocolate intake was similar in both groups, although it was expected to be smaller in the exposure group as compared to the control group. This may be due to the fact that extinction of craving was not attained in the chocolate exposure group, which may have been the result of the olfactory exposure being interrupted too often by scans.

In summary, all three regression analyses of our study found that neural correlates predicted subsequent behaviour (i.e., chocolate intake) above and beyond selfreported chocolate craving, which confirmed our hypothesis. The BOLD response in the caudate, which has been associated with long-term weight gain in previous studies, was a significant predictor of intake for the chocolate exposure group. The activation in the 
Table 4. Hierarchical multiple regression analysis predicting chocolate intake from self-reported craving and neural correlates. Variances explained $\left(\Delta R^{2}\right)$ by neural correlates are listed for comparative purposes only

\begin{tabular}{|c|c|c|}
\hline Predictor & $\Delta R^{2}$ & $\beta$ \\
\hline \multicolumn{3}{|l|}{ Whole sample $(n=34)$} \\
\hline Step 1: self-reported craving only & .04 & \\
\hline Optimal self-reported craving rating ${ }^{\ddagger}$ & & .19 \\
\hline Step 2: self-reported plus neural correlates & $.33^{* * *}$ & \\
\hline $\begin{array}{l}\text { Optimal self-reported craving rating } \\
\text { Activation in cluster A in run } 7\end{array}$ & & $\begin{array}{l}.13 \\
.58^{* * *}\end{array}$ \\
\hline Total $R^{2}$ & $.37^{* * *}$ & \\
\hline \multicolumn{3}{|l|}{ Exposure group $(n=17)$} \\
\hline Step 1: self-reported craving only & $.37^{* *}$ & \\
\hline Optimal self-reported craving rating ${ }^{+}$ & & $.61^{* *}$ \\
\hline Step 2: self-reported plus neural correlates & $.45^{* * *}$ & \\
\hline $\begin{array}{l}\text { Optimal self-reported craving rating } \\
\text { Activation in cluster B in run } 7 \\
\text { Activation in cluster C in run } 7\end{array}$ & & $\begin{array}{l}.32^{\star} \\
.58^{\star *} \\
.20\end{array}$ \\
\hline Total $R^{2}$ & $.82^{* * *}$ & \\
\hline \multicolumn{3}{|l|}{ Control group $(n=17)$} \\
\hline Step 1: self-reported craving only & .04 & \\
\hline Optimal self-reported craving rating ${ }^{\ddagger}$ & & -.20 \\
\hline Step 2: self-reported plus neural correlates & $.70^{* * *}$ & \\
\hline $\begin{array}{l}\text { Optimal self-reported craving rating }^{\ddagger} \\
\text { Activation in cluster } D \text { in run } 7 \\
\text { Activation in cluster } E \text { in run } 7\end{array}$ & & $\begin{array}{l}-.25 \\
-.20 \\
-.66^{*}\end{array}$ \\
\hline Total $R^{2}$ & $.74^{* * *}$ & \\
\hline
\end{tabular}

Note. Significance indications: ${ }^{*} p<.05 ;{ }^{* *} p<.01 ;{ }^{* *} p<.001$ (uncorrected).

${ }^{\ddagger}$ Highest correlation coefficient of the respective analysis between self-reported chocolate craving and intake, as reported in Table 2.

mid-dorsolateral PFC negatively predicted subsequent chocolate intake. This region has been linked to weight loss in previous studies. The response in the right anterior PFC significantly predicted intake in the whole sample. Lesions in this region have been associated with weight loss. Therefore, by providing an association with subsequent intake, our findings might provide for a missing link between brain activation and long-term weight change. Furthermore, only reward regions correlated with intake in the exposure group, and only inhibitory regions correlated with intake in the control group. In other words, 
there was a dominant influence of reward in the exposure group, and a dominant influence of inhibition on subsequent chocolate intake in the control group. In conclusion, neural correlates were more predictive of subsequent intake than was self-reported craving, for the chocolate exposure group, the control group and the whole sample. However, the brain regions found to be predictive differed between groups, both in location and in assumed functionality (reward in the exposure group versus inhibition in the control group). This can be considered as an effect of the CERP manipulation, which is noteworthy because of the fact that brain correlates were a better predictor of subsequent intake than self-reported craving. It remains to be investigated if, after an effective CERP, reward region activation would still be predictive of subsequent intake and if activation in reward regions would extinguish together with craving.

\section{ACKnowledgements}

We would like to thank Ing. Charlie Bonnemayer of Maastricht University for programming parts of the stimulus protocol in E-Prime, as well as Dr. Armin Heinecke of Brain Innovation B.V. for his help with the data analysis. This work was supported by the Netherlands Organisation for Scientific Research (NWO) (grant number 400.06.148 to Prof. Dr. Anita Jansen). 

Chapter 6

SUMMARY AND GENERAL DISCUSSION 
In the research presented in this dissertation, the influence of the type of attention (i.e., unbiased viewing without prior instructions versus focus on taste) on the neural processing of high-calorie palatable food cues was investigated in healthy-weight and overweight participants (Chapter 2). Furthermore, the effect of food-cue palatability on brain reward activity in overweight and healthy-weight participants was examined (Chapter 3). In addition, the effects of cue exposure with response prevention both on subjective craving for chocolate and on neural responses to chocolate pictures versus neutral pictures were measured over the course of a one-hour session (Chapter 4). Finally, the predictive characteristics of both subjective craving and brain activation in response to chocolate cues on subsequent chocolate intake were investigated (Chapter 5).

\section{RESEARCH QUESTIONS AND SUMMARY OF THE MAIN FINDINGS}

The aim of the first functional magnetic resonance imaging (fMRI) study (Chapter 2) was to examine reward-related brain activity in overweight versus healthy-weight participants in response to high-calorie palatable food pictures in two conditions of attention. In a first condition participants viewed the pictures without prior instructions (unbiased viewing), while in a taste imagination condition participants were instructed to imagine the taste of the presented food pictures. We predicted that neural activation in brain reward regions would be greater in overweight participants than in healthy-weight ones and that this effect would be most pronounced during unbiased viewing, because the group difference during taste imagination might be less pronounced as all participants would be evaluating food palatability and might thus show resembling brain reward activity. To this end, an event-related design was used, in which palatable and unpalatable high-calorie and low-calorie food pictures were presented during these two conditions (unbiased viewing vs. taste imagination). Participants were satiated overweight and healthy-weight women.

A whole-brain analysis was conducted, testing the group (overweight vs. healthy weight) $\times$ condition (unbiased viewing vs. taste imagination) interaction on the neural response specifically to high-calorie palatable food stimuli. This analysis resulted in 14 functional regions of interest (fROIs) involved in food reward processing. Neural activation in these regions was greater in the overweight participants than in the healthyweight ones during the taste imagination condition. During unbiased viewing, contrary to our expectations, the opposite pattern was observed: in the overweight participants the activation in reward regions was reduced compared to healthy-weight participants. This group $\times$ condition interaction was specific to high-calorie palatable food stimuli in all brain reward regions except for the left amygdala. This was apparent from group $\times$ condition interaction analyses on other types of food stimuli (i.e., high-calorie unpalatable and 
low-calorie palatable). These interaction analyses resulted in clusters that did not overlap with the 14 found fROIs for the high calorie palatable foods, except for the left amygdala. Therefore the remaining 13 fROIs can be considered unique to high-calorie palatable food stimuli. The fROIs that resulted from the interaction analyses on the other stimulus types were not considered further.

So, high-calorie palatable food cues elicited a greater reward response in overweight people than in healthy-weight people, but only during taste imagination. The reduced reward activation during unbiased viewing in overweight as compared to healthyweight people may reflect avoidance of processing of high-calorie palatable food stimuli in terms of how good they taste in overweight people, which is of course easier to achieve when the task does not require a focus on taste. Taken together, this pattern of activation might represent an ambivalence in the overweight group between desire for (in the taste imagination condition) and avoidance of (in the unbiased viewing condition) highcalorie palatable food stimuli.

In Chapter 3 it was investigated if overweight participants were more responsive to taste than were healthy-weight participants, both on a neural and a subjective level. Therefore the data acquired in Chapter 2 were re-analysed after pooling responses to high-calorie and low-calorie stimuli. Further, only responses to palatable and to neutral food cues were taken into account. It was expected that group differences would be found on a neural level, but not in subjective reports. In response to palatable versus neutral food cues, overweight participants as compared to healthy-weight ones were expected to show more reward-related brain activation, and less inhibitory activation. This group difference was expected to be more pronounced during taste imagination than during unbiased viewing.

The results contradicted our hypothesis when participants viewed the stimuli naturally without instructions (unbiased viewing): the response to palatable versus neutral food was smaller in overweight compared with healthy-weight participants in seven fROIs associated with reward. The opposite effect was found in three putative inhibitory fROIs: the response to palatable versus neutral food was larger in the overweight participants than in healthy-weight ones. Results were in line with our hypothesis during taste imagination. In this condition, in overweight compared with healthy-weight participants, seven reward-related fROIs were more active in response to palatable versus neutral food stimuli. These results show that when overweight participants view food stimuli naturally, their brain activation is very different from the activation when they focus on the taste of that food: in overweight people inhibitory processes are dominant during unbiased viewing, whereas reward-related processes are dominant during taste imagination. This study adds to the limited evidence-base that attention focus is highly relevant in the study of food reward and inhibition.

The study described in Chapter 4 investigated the role of cue exposure with response prevention (CERP) on subjective craving and on the neural processing of choco- 
late pictures versus neutral non-food pictures. The chocolate exposure group smelled chocolate, whereas the control group smelled a neutral control object (i.e., a wooden pencil). Cue exposure lasted one hour, during which participants were in the fMRI scanner. CERP was interrupted by seven short scanning sequences during which neutral and chocolate pictures were presented. In the exposure group, subjective chocolate craving was expected to increase until the middle of the session and from then on to decrease and extinguish at the end of the session. This craving curve was hypothesized to be mirrored by activation in brain reward regions.

As expected, a short exposure (30 $\mathrm{min}$ ) caused an increase in subjective chocolate craving in the exposure group, which was mirrored by a corresponding increase in brain reward activation in the exposure group as compared to the control group. Unexpectedly, a long exposure (60 min) did not lead to the extinction of subjective craving in the experimental group, although craving had started to decrease at that point. On a neural level however, activation in regions of interest in the exposure group seemed to have extinguished after the long exposure, because activation levels returned to or fell below control group levels. These results indicate that for a short exposure, brain reward activation during CERP is linked to craving. Regarding the longer exposure, the decline in brain reward activation in the experimental group may be a precursor of a decrease in subjective craving.

The aim of the study in Chapter $\mathbf{5}$ was to examine if brain regions could predict chocolate intake after participants had been either exposed to chocolate or to control stimuli during approximately one hour, and whether the variance in chocolate intake could be better explained by brain activation or by self-reported craving. Five brain regions in total correlated with subsequent chocolate intake. These consisted of two reward regions that correlated positively with intake in the exposure group, one of which was the right caudate. Further, two regions associated with cognitive control correlated negatively with intake in the control group. When the regression analysis was conducted with the exposure and the control group together, an additional region in the anterior prefrontal cortex correlated positively with chocolate intake. In all analyses, the intake variance explained by neural correlates was above and beyond the variance explained by self-reported craving. These results are in line with previous neuroimaging studies that showed that brain responses were a better predictor of subsequent behaviour than selfreported craving or intentions (Falk et al., 2010, 2011; Grüsser et al., 2004; Kosten et al., 2006). Therefore, our findings might provide a missing link by associating brain activation, previously shown to predict weight change, with short-term food intake. 


\section{GENERAL DISCUSSION AND CONCLUSIONS}

\section{Less reward activation during unbiased viewing in overweight than in healthy-weight people: an argument for attentional avoidance?}

Both in Chapters 2 and 3 it was found that, compared to healthy-weight participants, overweight participants showed more reward activation during taste imagination and less reward activation (and, additionally, in chapter 3 even inhibition) in the unbiased viewing condition. This difference between conditions shows how a focus of attention can modulate brain reward responses to food. The activation pattern during taste imagination was in line with our hypothesis and is thought to reflect a stronger motivation to approach or consume high-calorie palatable foods in overweight people, although participants were satiated.

A possible explanation for the reduced reward activation in overweight as compared to healthy-weight participants during unbiased viewing could be that during unbiased viewing, the overweight participants paid attention to health aspects, whereas the healthy-weight participants focused on the good taste of the high-calorie palatable foods. This focus on health may be due to higher scores of overweight participants on a measure of dietary restraint in this study as well as in previous studies (Herman \& Polivy, 1980; Van Strien et al., 2007). Similar results were observed in studies with restrained versus unrestrained women, who showed attentional avoidance of high-calorie food (Veenstra et al., 2010), and in overweight participants versus healthy-weight participants, who showed a reduced maintenance of attention on food pictures (Werthmann et al., 2011). This avoidance may have been aided by the fact that participants were tested while satiated. Maybe this avoidance cannot be sustained under disinhibiting circumstances like depression or distraction (Boon et al., 2002; Roefs et al., 2012), which have been shown to lead to infraction of restrictive intake norms and to overconsumption (Herman \& Polivy, 2007). Further, resources might become depleted over time by chronic self-regulation so that palatable, and often high-calorie, snack food becomes irresistible (Hofmann et al., 2007; Vohs \& Heatherton, 2000).

An explanation of the supposed avoidance could be the activation of inhibitory regions, which indeed was found in Chapter 3. It is not clear why there was no sign of inhibitory activation in Chapter 2. Most likely this was due to the type of analysis that was conducted: In Chapter 2 we only looked at high-calorie palatable stimuli and selected regions with an interaction between group and attention condition. In Chapter 3, both palatable and neutral food stimuli were taken into account and instead the interaction between group and stimulus type (palatable vs. neutral) was analysed. 


\section{The influence of cue exposure with response prevention (CERP)}

In Chapter 4 subjective craving for chocolate was found to increase after a short exposure to real chocolate, which was in accordance with our expectations. After a long exposure however, an extinction of subjective craving was not found, although a tendency towards a decrease in craving was significant at the end of the final prolonged exposure, which might have continued if the exposure had been even longer. Therefore it can be said that the CERP manipulation was not entirely successful, in that an extinction of subjective craving at the end of the session was not attained. This may have been due to the interruption of the olfactory exposure by six scan measurements, even though these measurements were short (only 5 min per scan). As discussed in Chapter 5, we suppose that as a result of this, chocolate intake in the exposure group was similar to that of the control group, although a smaller intake in the exposure group had been expected. If the CERP manipulation had been successful, this would probably have led to a decrease in chocolate intake in the exposure group, as would be expected from previous studies in which cue reactivity extinguished after a successful CERP (Van Gucht et al., 2008; Jansen, Broekmate, \& Heymans, 1992; Jansen, Van den Hout, De Loof, Zandbergen, \& Griez, 1989; Martinez-Mallén et al., 2007; Toro et al., 2003). This could be a subject of future studies. In Chapter 5 it was also shown that the CERP manipulation was influential with respect to the predictive validity of neural correlates of chocolate craving on intake, because the brain regions that predicted subsequent intake were different for each group, both in location and in assumed functionality (reward in the exposure group versus inhibition in the control group).

The comparison of subjective craving ratings and brain reward activation (Chapter 4) shows that for a short exposure, the changes in brain reward activation during CERP were linked to changes in craving. Therefore, a short cue exposure can be considered effective in increasing both craving and brain reward activation. For a longer exposure, however, there was a disaccord between these two measures at the end of the session: brain reward activation levels of the exposed group returned back to control group levels in fROIs associated with reward, whereas subjective chocolate craving was still higher in the exposure group than in the control group. A stricter analysis confirmed these findings. We can conclude that CERP is an effective intervention for the reduction of brain reward activation as a form of food cue reactivity, even though subjective craving did not extinguish in this study.

Interesting in the context of cue exposure is a study on the prevalence of obesity in 41 occupational groups, among which foodservice employees (Caban et al., 2005). It was found that foodservice employees were in general less often obese than employees with other occupations. This was surprising to the author of a recent study (Pizam, 2013), but in the light of the exposure studies of this dissertation this could be inter- 
preted as a continuous cue exposure to food and beverages, while eating and drinking often is not possible because either it is not allowed or there is no time for this in the foodservice industry. As a result, the association between the food and beverage cues and intake may be disrupted in these employees, leading to a lower prevalence of obesity in this occupational group.

\section{Prediction by brain activation is better than prediction by subjective reports}

The findings of Chapter 5 were that the variance in chocolate intake after the cue exposure session could be better explained by brain activation to chocolate cues than by selfreported chocolate craving. This is in line with previous neuroimaging studies showing that brain responses were a better predictor of subsequent behaviour than self-reported craving or intentions (Falk et al., 2010, 2011; Grüsser et al., 2004; Kosten et al., 2006). The reason for this could be that introspection is limited (Nisbett \& Wilson, 1977) and that self-reported craving may have become confounded by socially desirable answering tendencies (Schwarz \& Oyserman, 2001).

\section{Real food is a stronger cue than the image of that food}

In Chapter 4 it was found that the smell of real chocolate (during exposures) was more effective in increasing chocolate craving than the sight of chocolate pictures (during brain scans). This was indicated by a higher average of the six craving ratings after as compared with before each exposure to real chocolate in the chocolate exposure group. An opposite pattern was found in the control group: average chocolate craving ratings were lower after than before the pencil exposure, which indicated that the sight of chocolate pictures during the scan runs was more effective in increasing chocolate craving than the smell of a pencil. This is in line with previous literature, in that craving elicited by a real food cue and by mental imagery of that food cue are related (Kavanagh et al., 2005; Tiggemann \& Kemps, 2005). Our findings in the chocolate exposure group add to this literature by indicating that the perception of real food is a stronger cue than the image of that food with its accompanying imagined smell and taste. 


\section{Methodological issues}

Methodological issues are described in the discussion of every chapter, but the most important, as well as general issues, will be mentioned in the following.

\section{Choice of the design: event-related versus blocked}

The experiment described in Chapters 2 and 3 made use of an event-related design. This type of design is not very common in neuroimaging studies on food reward processing. The majority of studies in this field used a blocked design (e.g., Bruce et al., 2010; Killgore et al., 2003; Martens et al., 2013; Martin et al., 2010; McCaffery et al., 2009; Murdaugh, Cox, Cook, \& Weller, 2012; Rothemund et al., 2007; Stoeckel et al., 2008). The choice of our design may be the reason why the results of the studies described in Chapters 2 and 3 were partly contrary to our expectations and to previous research. For example, in Chapter 3 we found an activation of inhibitory control regions and a lack of activation of reward regions in overweight participants during unbiased (passive) viewing. The majority of studies on food reward with a condition of passive viewing without instructions found the opposite, that is, more activation in reward regions in overweight compared with healthy-weight participants (e.g., Bruce et al., 2010; Martens et al., 2013; Murdaugh, Cox, Cook, \& Weller, 2012; Rothemund et al., 2007; Stoeckel et al., 2008). The reason for this discrepancy could be the type of design: a blocked design in the previous studies versus an event-related design in our study. In a blocked design, multiple palatable food stimuli are shown consecutively. Therefore, craving for the shown food could be stronger in this type of design than in our event-related design in which stimuli of different categories are mixed. Even in a condition of passive viewing without instructions, a blocked design may result in a stronger build-up of craving because of the grouped presentation of high-calorie palatable foods. This would overrule any natural inhibitory response to a single stimulus and this may be the reason why no such responses were found in the majority of food reward studies.

Because in an event-related design the reward value of individual food stimuli is assessed, the measurement of responses to food stimuli in an event-related design gives, in our opinion, more ecologically valid results than measurement in a blocked design. This type of design seems to be closer to reality than a blocked design, because in real life people also view stimuli of different categories almost simultaneously or consecutively (e.g., a food stimulus next to a pair of scissors and a picture postcard on a colourful table cloth). In a blocked design one can only measure the brain activity in response to a group of stimuli. Such settings also occur in real life (e.g., a buffet dinner table, or the supermarket corridor with shelves full of snack food), but less often, because food intake takes mostly place at home. 


\section{Measurement of neural responses during satiation instead of hunger}

Both experiments (Chapters 2 to 5 ) have been conducted with participants who had eaten lunch before they were tested. We chose to test satiated instead of hungry participants because the excess of energy intake in obesity is at least partly due to eating in the absence of hunger, during which hedonic drives override homeostatic ones (Berthoud, 2011; Fisher \& Birch, 2002; Zheng et al., 2009). Therefore we expected that the difference in neural food reward processing between healthy-weight and overweight participants would be found primarily in a state of satiation. Together with the event-related (instead of blocked) design, this may have contributed to our findings of less reward activation in overweight versus healthy-weight participants during unbiased viewing (Chapters 2 and 3), which differ from previous studies. These previous studies found opposite results (i.e., more reward activation in overweight versus healthy-weight participants), but these participants were tested in a hungry state (e.g., Murdaugh, Cox, Cook, \& Weller, 2012; Stice, Spoor, Bohon, Veldhuizen, \& Small, 2008; Stoeckel et al., 2008).

\section{Limitations of "unbiased viewing"}

One of the conditions in the first experiment (Chapters 2 and 3) was unbiased viewing, that is, viewing of the food stimuli without prior instructions. It was assumed that this is the most natural way of presentation of food pictures to participants. However, one may argue that the environment in which the brain responses to these stimuli are recorded is not natural at all: the participant is lying supine in the scanner and is not allowed to move, while noise levels are very high and communication with the researcher is not possible other than by a microphone and an alarm button. Therefore, brain activation in response to real food in a supermarket or a restaurant may differ from brain activation that is recorded during an fMRI session. This drawback is common to all fMRI experiments. An advantage of our first experiment compared to other fMRI studies is that our experiment contained a task (in both the unbiased viewing and the taste imagination condition). In many other studies involving passive viewing, no task is given. Therefore, in those studies it remains largely unknown what participants have been thinking about during the presentation of stimuli, even if this had to be indicated in an exit questionnaire.

\section{Participant characteristics}

In both experiments (Chapters 2 to 5), only female participants were selected to take part, because men and women seem to differ regarding food reward processing both behaviourally (Havermans, Giesen, Houben, \& Jansen, 2011) and neurally (Frank et al., 2010; Haase et al., 2011; Uher et al., 2006; Wang et al., 2009). It remains to be investigated 
what the neural reaction of male participants would be. Furthermore, in the first experiment (Chapters 2 and 3), only four of the participants in the overweight group could be considered obese. Results may have differed if the whole group had been obese instead of overweight, because there is evidence that the relationship between sensitivity to reward and body mass index (BMI) is of an inverse U-shape (Davis \& Fox, 2008), which suggests that brain responses to food stimuli in regions associated with reward might differ in overweight versus obese participants.

\section{Comparison of results with previous fMRI-studies}

For the interpretation of the results of the studies in this dissertation regarding brain activation it was necessary to compare them with previous neuroimaging studies. This comparison turned out to be laborious, as there was a large variation in the naming of brain regions, even if the studies meant the same brain region. For example, a region in the lateral prefrontal cortex can be called "pars triangularis", "Brodmann Area 45", "inferior frontal gyrus" or "ventrolateral prefrontal cortex", depending on the level of precision which is employed by the authors. These varying denominations made it very timeconsuming to systematically search previous papers for results in brain regions similar to the ones found in our experiments. Additionally, the indications of the peak voxel coordinates of the brain region can be given in different coordinate systems, the comparison of which requires an extra conversion step, such as Talairach coordinates (used in this thesis) and Montreal Neurological Institute (MNI) coordinates. Comparison of Talairach and MNI coordinates can only be done after one of the coordinates has been converted into the other system. Apart from this requiring an extra conversion step, a coordinate disparity of around 1 to $2 \mathrm{~mm}$ will remain due to the different scaling, orientation and origin of reference brains of the two coordinate systems (Lancaster et al., 2007). A standardisation of the naming of brain regions, together with a uniform coordinate system which is used for the localisation of voxels, would have been very helpful. Finally, the results of the tool which we used to automatically localize brain regions (Talairach Client (www.talairach.org) (Lancaster et al., 1997, 2000)) regularly needed to be corrected manually because several brain regions determined by the Talairach Client clearly differed from the activated brain regions in our analyses. 
Appendix 



\section{REFERENCES}

Appelhans, B. M. (2009). Neurobehavioral inhibition of reward-driven feeding: implications for dieting and obesity. Obesity (Silver Spring, Md.), 17(4), 640-7. doi:10.1038/oby.2008.638

Arana, F. S., Parkinson, J. A., Hinton, E., Holland, A. J., Owen, A. M., \& Roberts, A. C. (2003). Dissociable contributions of the human amygdala and orbitofrontal cortex to incentive motivation and goal selection. The Journal of neuroscience: the official journal of the Society for Neuroscience, 23(29), 9632-8.

Asterholm, I. W., \& Scherer, P. E. (2012). Metabolic jet lag when the fat clock is out of sync. Nature Medicine, 18(12), 1738-1740. doi:10.1038/nm.3010

Bandettini, P. (2012). Twenty years of functional MRI: the science and the stories. NeuroImage, 62(2), 57588. doi:10.1016/j.neuroimage.2012.04.026

Batterink, L., Yokum, S., \& Stice, E. (2010). Body mass correlates inversely with inhibitory control in response to food among adolescent girls: An fMRI study. NeuroImage, 52(4), 1696-1703. doi:10.1016/j. neuroimage.2010.05.059

Beaver, J. D., Van Ditzhuijzen, J., Lawrence, A. D., Davis, M. H., Woods, A., \& Calder, A. J. (2006). Individual differences in reward drive predict neural responses to images of food. The Journal of neuroscience: the official journal of the Society for Neuroscience, 26(19), 5160-6. doi:10.1523/JNEUROSCI.0350-06.2006

Belliveau, J. W., Kennedy, D. N., McKinstry, R. C., Buchbinder, B. R., Weisskoff, R. M., Cohen, M. S., Vevea, J. M., et al. (1991). Functional mapping of the human visual cortex by magnetic resonance imaging. Science (New York, N.Y.), 254(5032), 716-9.

Bender, G., Veldhuizen, M. G., Meltzer, J. A., Gitelman, D. R., \& Small, D. M. (2009). Neural correlates of evaluative compared with passive tasting. The European journal of neuroscience, 30(2), 327-38. doi:10.1111/j.1460-9568.2009.06819.x

Benton, D., Greenfield, K., \& Morgan, M. (1998). The development of the attitudes to chocolate questionnaire. Personality and Individual Differences, 24(4), 513-520.

Berghöfer, A., Pischon, T., Reinhold, T., Apovian, C. M., Sharma, A. M., \& Willich, S. N. (2008). Obesity prevalence from a European perspective: a systematic review. BMC public health, 8, 200. doi:10.1186/1471-2458-8-200 
Berridge, K. C. (1996). Food reward: brain substrates of wanting and liking. Neuroscience and biobehavioral reviews, 20(1), 1-25. doi:10.1016/0149-7634(95)00033-B

Berridge, K. C. (2004). Motivation concepts in behavioral neuroscience. Physiology \& behavior, 81(2), 179209. doi:10.1016/j.physbeh.2004.02.004

Berridge, K. C., Ho, C.-Y., Richard, J. M., \& DiFeliceantonio, A. G. (2010). The tempted brain eats: pleasure and desire circuits in obesity and eating disorders. Brain research, 1350, 43-64. doi:10.1016/j. brainres.2010.04.003

Berthoud, H.-R. (2011). Metabolic and hedonic drives in the neural control of appetite: who is the boss? Current opinion in neurobiology, 21(6), 888-96. doi:10.1016/j.conb.2011.09.004

Berthoud, H.-R., Lenard, N. R., \& Shin, A. C. (2011). Food reward, hyperphagia, and obesity. American journal of physiology. Regulatory, integrative and comparative physiology, 300(6), R1266-77. doi:10.1152/ajpregu.00028.2011

Boggiano, M. M., Dorsey, J. R., Thomas, J. M., \& Murdaugh, D. L. (2009). The Pavlovian power of palatable food: lessons for weight-loss adherence from a new rodent model of cue-induced overeating. International Journal of Obesity, 33(6), 693-701. doi:10.1038/ijo.2009.57

Bond, D. S., Raynor, H. a, Vithiananthan, S., Sax, H. C., Pohl, D., Roye, G. D., Ryder, B. a, et al. (2009). Differences in salivary habituation to a taste stimulus in bariatric surgery candidates and normal-weight controls. Obesity surgery, 19(7), 873-8. doi:10.1007/s11695-009-9861-3

Boon, B., Stroebe, W., Schut, H., \& Ijntema, R. (2002). Ironic processes in the eating behaviour of restrained eaters. British journal of health psychology, 7(Pt 1), 1-10. doi:10.1348/135910702169303

Boorman, E. D., Behrens, T. E. J., Woolrich, M. W., \& Rushworth, M. F. S. (2009). How green is the grass on the other side? Frontopolar cortex and the evidence in favor of alternative courses of action. Neuron, 62(5), 733-43. doi:10.1016/j.neuron.2009.05.014

BrainInnovation (2001). BrainVoyager QX (Version 2.4.1.2052, 32-bit) [Computer software]. Maastricht, The Netherlands: Brain Innovation. Retrieved from http://www.brainvoyager.com.

Bruce, A. S., Holsen, L. M., Chambers, R. J., Martin, L. E., Brooks, W. M., Zarcone, J. R., Butler, M. G., et al. (2010). Obese children show hyperactivation to food pictures in brain networks linked to motivation, reward and cognitive control. International Journal of Obesity, 34(10), 1494-500. doi:10.1038/ijo.2010.84

Bruce, B., \& Agras, W. S. (1992). Binge eating in females: A population-based investigation. International Journal of Eating Disorders, 12(4), 365-373. doi:10.1002/1098-108X(199212)12:4<365::AIDEAT2260120404>3.0.CO;2-M

Bryant, M., Truesdale, K. P., \& Dye, L. (2006). Modest changes in dietary intake across the menstrual cycle: implications for food intake research. The British journal of nutrition, 96(5), 888-94. doi:10.1017/ BJN20061931

Caban, A. J., Lee, D. J., Fleming, L. E., Gómez-Marín, O., LeBlanc, W., \& Pitman, T. (2005). Obesity in US workers: The National Health Interview Survey, 1986 to 2002. American journal of public health, 95(9), 1614-22. doi:10.2105/AJPH.2004.050112

Carnell, S., Gibson, C., Benson, L., Ochner, C. N., \& Geliebter, A. (2012). Neuroimaging and obesity: current knowledge and future directions. Obesity reviews: an official journal of the International Association for the Study of Obesity, 13(1), 43-56. doi:10.1111/j.1467-789X.2011.00927.x 
Carter, F. A., McIntosh, V. V. W., Joyce, P. R., Frampton, C. M. A., \& Bulik, C. M. (2006). Cue reactivity in bulimia nervosa: A useful self-report approach. The International journal of eating disorders, 39(8), 694-9. doi:10.1002/eat.20331

CBS (2012a). Landelijke Jeugdmonitor - Jaarrapport 2012. Den Haag: Centraal Bureau voor de Statistiek.

CBS (2012b). Gezondheid en zorg in cijfers 2012. Den Haag: Centraal Bureau voor de Statistiek.

CBS (2012c). Smakelijk weten. Trends in voeding en gezondheid. Den Haag: Centraal Bureau voor de Statistiek.

Choi, J.-S., Park, S., Lee, J.-Y., Jung, H.-Y., Lee, H.-W., Jin, C.-H., \& Kang, D.-H. (2011). The effect of repeated virtual nicotine cue exposure therapy on the psychophysiological responses: a preliminary study. Psychiatry investigation, 8(2), 155-60. doi:10.4306/pi.2011.8.2.155

Coelho, J. S., Nederkoorn, C., \& Jansen, A. (2013). Acute versus Repeated Chocolate Exposure: Effects on Intake and Cravings in Restrained and Unrestrained Eaters. Journal of health psychology. doi:10.1177/1359105312473787

Coletta, M., Platek, S., Mohamed, F. B., Van Steenburgh, J. J., Green, D., \& Lowe, M. R. (2009). Brain activation in restrained and unrestrained eaters: an fMRI study. Journal of abnormal psychology, 118(3), 598-609. doi:10.1037/a0016201

Conklin, C. A., \& Tiffany, S. T. (2002). Applying extinction research and theory to cue-exposure addiction treatments. Addiction (Abingdon, England), 97(2), 155-67.

Cornier, M.-A., Rojas, D. C., Salzberg, A. K., Endly, D. C., Bessesen, D. H., \& Tregellas, J. R. (2009). The effects of overfeeding on the neuronal response to visual food cues in thin and reduced-obese individuals. PloS one, 4(7), e6310. doi:10.1371/journal.pone.0006310

Coutlee, C. G., \& Huettel, S. A. (2012). The functional neuroanatomy of decision making: prefrontal control of thought and action. Brain research, 1428, 3-12. doi:10.1016/j.brainres.2011.05.053

Craig, A. D. B. (2004). Human feelings: why are some more aware than others? Trends in cognitive sciences, 8(6), 239-41. doi:10.1016/j.tics.2004.04.004

Craig, A. D. B. (2009). How do you feel--now? The anterior insula and human awareness. Supplementary information. Nature reviews. Neuroscience, 10(1), 59-70. doi:10.1038/nrn2555

Cramer, K., \& Hartleib, M. (2001). The attitudes to chocolate questionnaire: a psychometric evaluation. Personality and Individual Differences, 31(6), 931-942. doi:10.1016/S0191-8869(00)00195-1

Custers, R., \& Aarts, H. (2010). The unconscious will: how the pursuit of goals operates outside of conscious awareness. Science (New York, N.Y.), 329(5987), 47-50. doi:10.1126/science.1188595

Davis, C., \& Fox, J. (2008). Sensitivity to reward and body mass index (BMI): evidence for a non-linear relationship. Appetite, 50(1), 43-9. doi:10.1016/j.appet.2007.05.007

Davis, C., Patte, K., Levitan, R., Reid, C., Tweed, S., \& Curtis, C. (2007). From motivation to behaviour: a model of reward sensitivity, overeating, and food preferences in the risk profile for obesity. Appetite, 48(1), 12-9. doi:10.1016/j.appet.2006.05.016

De Zwaan, M., \& Mitchell, J. E. (1992). Binge eating in the obese. Annals of medicine, 24(4), 303-8.

Deichmann, R., Good, C. D., Josephs, O., Ashburner, J., \& Turner, R. (2000). Optimization of 3-D MP-RAGE sequences for structural brain imaging. NeuroImage, 12(1), 112-27. doi:10.1006/nimg.2000.0601 
Deichmann, R., Gottfried, J. A., Hutton, C., \& Turner, R. (2003). Optimized EPI for fMRI studies of the orbitofrontal cortex. NeuroImage, 19(2 Pt 1), 430-41. doi:10.1016/S1053-8119(03)00073-9

DelParigi, A., Chen, K., Salbe, A. D., Hill, J. O., Wing, R. R., Reiman, E. M., \& Tataranni, P. A. (2007). Successful dieters have increased neural activity in cortical areas involved in the control of behavior. International Journal of Obesity, 31(3), 440-8. doi:10.1038/sj.ijo.0803431

Demos, K. E., Heatherton, T. F., \& Kelley, W. M. (2012). Individual differences in nucleus accumbens activity to food and sexual images predict weight gain and sexual behavior. The Journal of neuroscience: the official journal of the Society for Neuroscience, 32(16), 5549-52. doi:10.1523/JNEUROSCI.5958-11.2012

Dimitropoulos, A., Tkach, J., Ho, A., \& Kennedy, J. (2012). Greater corticolimbic activation to high-calorie food cues after eating in obese vs. normal-weight adults. Appetite, 58(1), 303-12. doi:10.1016/j.appet.2011.10.014

DiQuattro, N. E., \& Geng, J. J. (2011). Contextual knowledge configures attentional control networks. The Journal of neuroscience: the official journal of the Society for Neuroscience, 31(49), 18026-35. doi:10.1523/JNEUROSCI.4040-11.2011

Dreher, J.-C., Schmidt, P. J., Kohn, P., Furman, D., Rubinow, D., \& Berman, K. F. (2007). Menstrual cycle phase modulates reward-related neural function in women. Proceedings of the National Academy of Sciences of the United States of America, 104(7), 2465-70. doi:10.1073/pnas.0605569104

Drewnowski, A. (2007). The real contribution of added sugars and fats to obesity. Epidemiologic reviews, 29, 160-71. doi:10.1093/epirev/mxm011

Epstein, L. H., \& Leddy, J. J. (2006). Food reinforcement. Appetite, 46(1), 22-5. doi:10.1016/j.appet.2005.04.006

Epstein, L. H., Leddy, J. J., Temple, J. L., \& Faith, M. S. (2007). Food reinforcement and eating: a multilevel analysis. Psychological bulletin, 133(5), 884-906. doi:10.1037/0033-2909.133.5.884

Epstein, L. H., Robinson, J. L., Temple, J. L., Roemmich, J. N., Marusewski, A., \& Nadbrzuch, R. (2008). Sensitization and habituation of motivated behavior in overweight and non-overweight children. Learning

Epstein, L. H., Temple, J. L., Neaderhiser, B. J., Salis, R. J., Erbe, R. W., \& Leddy, J. J. (2007). Food reinforcement, the dopamine D2 receptor genotype, and energy intake in obese and nonobese humans. Behavioral neuroscience, 121(5), 877-86. doi:10.1037/0735-7044.121.5.877

Falk, E. B., Berkman, E. T., Mann, T., Harrison, B., \& Lieberman, M. D. (2010). Predicting persuasioninduced behavior change from the brain. The Journal of neuroscience: the official journal of the Society for Neuroscience, 30(25), 8421-4. doi:10.1523/JNEUROSCI.0063-10.2010

Falk, E. B., Berkman, E. T., Whalen, D., \& Lieberman, M. D. (2011). Neural activity during health messaging predicts reductions in smoking above and beyond self-report. Health psychology: official journal of the Division of Health Psychology, American Psychological Association, 30(2), 177-85. doi:10.1037/a0022259

Ferriday, D., \& Brunstrom, J. M. (2011). “I just can”t help myself': effects of food-cue exposure in overweight and lean individuals. International Journal of Obesity, 35(1), 142-9. doi:10.1038/ijo.2010.117

Fisher, J. O., \& Birch, L. L. (2002). Eating in the absence of hunger and overweight in girls from 5 to $7 \mathrm{y}$ of age. The American journal of clinical nutrition, 76(1), 226-31. 
Forman, S. D., Cohen, J. D., Fitzgerald, M., Eddy, W. F., Mintun, M. A., \& Noll, D. C. (1995). Improved assessment of significant activation in functional magnetic resonance imaging (fMRI): use of a clustersize threshold. Magnetic resonance in medicine: official journal of the Society of Magnetic Resonance in Medicine / Society of Magnetic Resonance in Medicine, 33(5), 636-47.

Fox, M. D., Snyder, A. Z., Vincent, J. L., Corbetta, M., Van Essen, D. C., \& Raichle, M. E. (2005). The human brain is intrinsically organized into dynamic, anticorrelated functional networks. Proceedings of the National Academy of Sciences of the United States of America, 102(27), 9673-8. doi:10.1073/ pnas.0504136102

Frank, S., Laharnar, N., Kullmann, S., Veit, R., Canova, C., Hegner, Y. L., Fritsche, A., et al. (2010). Processing of food pictures: influence of hunger, gender and calorie content. Brain research, 1350, 159-66. doi:10.1016/j.brainres.2010.04.030

Franken, I. H. A., \& Muris, P. (2005). Individual differences in reward sensitivity are related to food craving and relative body weight in healthy women. Appetite, 45(2), 198-201. doi:10.1016/j.appet.2005.04.004

Frankort, A., Roefs, A., Siep, N., Roebroeck, A., Havermans, R., \& Jansen, A. (2012). Reward activity in satiated overweight women is decreased during unbiased viewing but increased when imagining taste: an event-related fMRI study. International Journal of Obesity, 36(5), 627-37. doi:10.1038/ijo.2011.213

Frankort, A., Roefs, A., Siep, N., Roebroeck, A., Havermans, R., \& Jansen, A. (2013). The Craving Stops Before You Feel It: Neural Correlates of Chocolate Craving During Cue Exposure with Response Prevention. Cerebral Cortex. doi:10.1093/cercor/bht016

Friedman, M. I., Ulrich, P., \& Mattes, R. D. (1999). A figurative measure of subjective hunger sensations. Appetite, 32(3), 395-404. doi:10.1006/appe.1999.0230

Friston, K. J., Fletcher, P., Josephs, O., Holmes, A., Rugg, M. D., \& Turner, R. (1998). Event-related fMRI: characterizing differential responses. NeuroImage, 7(1), 30-40. doi:10.1006/nimg.1997.0306

Führer, D., Zysset, S., \& Stumvoll, M. (2008). Brain activity in hunger and satiety: an exploratory visually stimulated FMRI study. Obesity (Silver Spring, Md.), 16(5), 945-50. doi:10.1038/oby.2008.33

García-García, I., Jurado, M. Á., Garolera, M., Segura, B., Marqués-Iturria, I., Pueyo, R., Vernet-Vernet, M., et al. (2013). Functional connectivity in obesity during reward processing. NeuroImage, 66, 232-239. doi:10.1016/j.neuroimage.2012.10.035

García-García, I., Narberhaus, A., Marqués-Iturria, I., Garolera, M., Rădoi, A., Segura, B., Pueyo, R., et al. (2013). Neural responses to visual food cues: insights from functional magnetic resonance imaging. European eating disorders review: the journal of the Eating Disorders Association, 21(2), 89-98. doi:10.1002/erv.2216

Gauggel, S., Heusinger, A., Forkmann, T., Boecker, M., Lindenmeyer, J., Cox, W. M., \& Staedtgen, M. (2010). Effects of alcohol cue exposure on response inhibition in detoxified alcohol-dependent patients. $\mathrm{Al}$ coholism, clinical and experimental research, 34(9), 1584-9. doi:10.1111/j.1530-0277.2010.01243.x

Gearhardt, A. N., Yokum, S., Orr, P. T., Stice, E., Corbin, W. R., \& Brownell, K. D. (2011). Neural correlates of food addiction. Archives of general psychiatry, 68(8), 808-16. doi:10.1001/archgenpsychiatry.2011.32

Giesen, J. C. A. H., Havermans, R. C., Douven, A., Tekelenburg, M., \& Jansen, A. (2010). Will work for snack food: the association of BMI and snack reinforcement. Obesity (Silver Spring, Md.), 18(5), 966-70. doi:10.1038/oby.2010.20 
Goebel, R., Esposito, F., \& Formisano, E. (2006). Analysis of functional image analysis contest (FIAC) data with brainvoyager QX: From single-subject to cortically aligned group general linear model analysis and self-organizing group independent component analysis. Human brain mapping, 27(5), 392-401. doi:10.1002/hbm.20249

Gottfried, J. A., O’Doherty, J. P., \& Dolan, R. J. (2003). Encoding predictive reward value in human amygdala and orbitofrontal cortex. Science (New York, N.Y.), 301(5636), 1104-7. doi:10.1126/science.1087919

Greenberg, A. S., Esterman, M., Wilson, D., Serences, J. T., \& Yantis, S. (2010). Control of spatial and feature-based attention in frontoparietal cortex. The Journal of neuroscience: the official journal of the Society for Neuroscience, 30(43), 14330-9. doi:10.1523/JNEUROSCI.4248-09.2010

Grüsser, S. M., Wrase, J., Klein, S., Hermann, D., Smolka, M. N., Ruf, M., Weber-Fahr, W., et al. (2004). Cueinduced activation of the striatum and medial prefrontal cortex is associated with subsequent relapse in abstinent alcoholics. Psychopharmacology, 175(3), 296-302. doi:10.1007/s00213-004-1828-4

Guerrieri, R., Nederkoorn, C., \& Jansen, A. (2008). The interaction between impulsivity and a varied food environment: its influence on food intake and overweight. International Journal of Obesity, 32(4), 708-14. doi:10.1038/sj.ijo.0803770

Guillebaud, J. (1987). The mechanism of the pill. In A. D. G. Gunn (Ed.), Oral Contraception in Perspective. Thirty Years of Clinical Experience with the Pill. (pp. 75-84). New Jersey, USA: The Parthenon Publishing Group.

Haase, L., Green, E., \& Murphy, C. (2011). Males and females show differential brain activation to taste when hungry and sated in gustatory and reward areas. Appetite, 57(2), 421-434. doi:10.1016/j.appet.2011.06.009

Harris, J. L., Bargh, J. a, \& Brownell, K. D. (2009). Priming effects of television food advertising on eating behavior. Health psychology: official journal of the Division of Health Psychology, American Psychological Association, 28(4), 404-13. doi:10.1037/a0014399

Havermans, R., Giesen, J. C. a H., Houben, K., \& Jansen, A. (2011). Weight, gender, and snack appeal. Eating behaviors, 12(2), 126-30. doi:10.1016/j.eatbeh.2011.01.010

Havermans, R., \& Jansen, A. (2003). Increasing the efficacy of cue exposure treatment in preventing relapse of addictive behavior. Addictive behaviors, 28(5), 989-94. doi:10.1016/S0306-4603(01)00289-1

Hepworth, R., Mogg, K., Brignell, C., \& Bradley, B. P. (2010). Negative mood increases selective attention to food cues and subjective appetite. Appetite, 54(1), 134-42. doi:10.1016/j.appet.2009.09.019

Herbert, B. M., \& Pollatos, O. (2012). The body in the mind: on the relationship between interoception and embodiment. Topics in cognitive science, 4(4), 692-704. doi:10.1111/j.1756-8765.2012.01189.x

Herman, C. P., \& Polivy, J. (1980). Restrained eating. In A. J. Stunkard (Ed.), Obesity (pp. 208-225). Philadelphia: Saunders.

Herman, C. P., \& Polivy, J. (2007). Norm-violation, norm-adherence, and overeating. Collegium antropologicum, 31(1), 55-62.

Herman, C. P., Roth, D. A., \& Polivy, J. (2003). Effects of the presence of others on food intake: a normative interpretation. Psychological bulletin, 129(6), 873-86. doi:10.1037/0033-2909.129.6.873 
Hermans, R. C. J., Larsen, J. K., Herman, C. P., \& Engels, R. C. M. E. (2012). How much should I eat? Situational norms affect young women's food intake during meal time. The British journal of nutrition, 107(4), 588-94. doi:10.1017/S0007114511003278

Higgs, S., \& Jones, A. (2013). Prolonged chewing at lunch decreases later snack intake. Appetite, 62, 91-5. doi:10.1016/j.appet.2012.11.019

Higgs, S., Williamson, A. C., Rotshtein, P., \& Humphreys, G. W. (2008). Sensory-specific satiety is intact in amnesics who eat multiple meals. Psychological science, 19(7), 623-8. doi:10.1111/j.14679280.2008.02132.x

Higgs, S., \& Woodward, M. (2009). Television watching during lunch increases afternoon snack intake of young women. Appetite, 52(1), 39-43. doi:10.1016/j.appet.2008.07.007

Hill, A. J. (2007). The psychology of food craving. The Proceedings of the Nutrition Society, 66(2), 277-85. doi:10.1017/S0029665107005502

Hill, J. O., Catenacci, V., \& Wyatt, H. R. (2005). Obesity: overview of an epidemic. The Psychiatric clinics of North America, 28(1), 1-23, vii. doi:10.1016/j.psc.2004.09.010

Ho, A., Kennedy, J., \& Dimitropoulos, A. (2012). Neural correlates to food-related behavior in normalweight and overweight/obese participants. PloS one, 7(9), e45403. doi:10.1371/journal.pone.0045403

Hofmann, W., Rauch, W., \& Gawronski, B. (2007). And deplete us not into temptation: Automatic attitudes, dietary restraint, and self-regulatory resources as determinants of eating behavior. Journal of Experimental Social Psychology, 43(3), 497-504. doi:10.1016/j.jesp.2006.05.004

Holsen, L. M., Zarcone, J. R., Thompson, T. I., Brooks, W. M., Anderson, M. F., Ahluwalia, J. S., Nollen, N. L., et al. (2005). Neural mechanisms underlying food motivation in children and adolescents. NeuroImage, 27(3), 669-76. doi:10.1016/j.neuroimage.2005.04.043

Hutcherson, C., Plassmann, H., Gross, J. J., \& Rangel, A. (2012). Cognitive Regulation during Decision Making Shifts Behavioral Control between Ventromedial and Dorsolateral Prefrontal Value Systems. The Journal of neuroscience: the official journal of the Society for Neuroscience, 32(39), 13543-54. doi:10.1523/JNEUROSCI.6387-11.2012

Jansen, A. (1998). A learning model of binge eating: cue reactivity and cue exposure. Behaviour research and therapy, 36(3), 257-72.

Jansen, A., Broekmate, J., \& Heymans, M. (1992). Cue-exposure vs self-control in the treatment of binge eating: a pilot study. Behaviour research and therapy, 30(3), 235-41.

Jansen, A., Havermans, R., \& Nederkoorn, C. (2011). Cued Overeating. In V. R. Preedy, R. R. Watson, \& C. R. Martin (Eds.), Handbook of Behavior, Food and Nutrition (pp. 1431-1443). New York, NY: Springer New York. doi:10.1007/978-0-387-92271-3

Jansen, A., Stegerman, S., Roefs, A., Nederkoorn, C., \& Havermans, R. (2010). Decreased salivation to food cues in formerly obese successful dieters. Psychotherapy and psychosomatics, 79(4), 257-8. doi: $10.1159 / 000315131$

Jansen, A., Theunissen, N., Slechten, K., Nederkoorn, C., Boon, B., Mulkens, S., \& Roefs, A. (2003). Overweight children overeat after exposure to food cues. Eating behaviors, 4(2), 197-209. doi:10.1016/ S1471-0153(03)00011-4 
Jansen, A., Van den Hout, M. A., De Loof, C., Zandbergen, J., \& Griez, E. (1989). A case of bulimia successfully treated by cue exposure. Journal of behavior therapy and experimental psychiatry, 20(4), 327-32.

Kavanagh, D. J., Andrade, J., \& May, J. (2005). Imaginary relish and exquisite torture: the elaborated intrusion theory of desire. Psychological review, 112(2), 446-67. doi:10.1037/0033-295X.112.2.446

Kaye, W. H., Fudge, J. L., \& Paulus, M. P. (2009). New insights into symptoms and neurocircuit function of anorexia nervosa. Nature reviews. Neuroscience, 10(8), 573-84. doi:10.1038/nrn2682

Kelly, T., Yang, W., Chen, C.-S., Reynolds, K., \& He, J. (2008). Global burden of obesity in 2005 and projections to 2030. International Journal of Obesity, 32(9), 1431-7. doi:10.1038/ijo.2008.102

Killgore, W. D. S., Schwab, Z. J., Weber, M., Kipman, M., Deldonno, S. R., Weiner, M. R., \& Rauch, S. L. (2013). Daytime sleepiness affects prefrontal regulation of food intake. NeuroImage, 71C, 216-223. doi:10.1016/j.neuroimage.2013.01.018

Killgore, W. D. S., Young, A. D., Femia, L. A., Bogorodzki, P., Rogowska, J., \& Yurgelun-Todd, D. A. (2003). Cortical and limbic activation during viewing of high- versus low-calorie foods. NeuroImage, 19(4), 1381-94. doi:10.1016/S1053-8119(03)00191-5

Killgore, W. D. S., \& Yurgelun-Todd, D. A. (2006). Affect modulates appetite-related brain activity to images of food. The International journal of eating disorders, 39(5), 357-63. doi:10.1002/eat.20240

Kishinevsky, F. I., Cox, J. E., Murdaugh, D. L., Stoeckel, L. E., Cook, E. W., \& Weller, R. E. (2012). fMRI reactivity on a delay discounting task predicts weight gain in obese women. Appetite, 58(2), 582-92. doi:10.1016/j.appet.2011.11.029

Kober, H., Mende-Siedlecki, P., Kross, E. F., Weber, J., Mischel, W., Hart, C. L., \& Ochsner, K. N. (2010). Prefrontal-striatal pathway underlies cognitive regulation of craving. Proceedings of the National Academy of Sciences of the United States of America, 107(33), 14811-6. doi:10.1073/pnas.1007779107 Koppelstaetter, F., Poeppel, T. D., Siedentopf, C. M., Ischebeck, A., Kolbitsch, C., Mottaghy, F. M., Felber, S. R., et al. (2010). Caffeine and cognition in functional magnetic resonance imaging. Journal of Alzheimer's disease: JAD, 20 Suppl 1, S71-84. doi:10.3233/JAD-2010-1417

Kosten, T. R., Scanley, B. E., Tucker, K. A., Oliveto, A., Prince, C., Sinha, R., Potenza, M. N., et al. (2006). Cue-induced brain activity changes and relapse in cocaine-dependent patients. Neuropsychopharmacology: official publication of the American College of Neuropsychopharmacology, 31(3), 644-50. doi:10.1038/sj.npp.1300851

Kringelbach, M. L. (2009). The hedonic brain: a functional neuroanatomy of human pleasure. In M. L. Kringelbach \& K. C. Berridge (Eds.), Pleasures of the brain (1st ed., pp. 202-221). New York: Oxford University Press.

Kringelbach, M. L., De Araujo, I. E., \& Rolls, E. T. (2004). Taste-related activity in the human dorsolateral prefrontal cortex. NeuroImage, 21(2), 781-8. doi:10.1016/j.neuroimage.2003.09.063

LaBar, K. S., Gitelman, D. R., Parrish, T. B., Kim, Y. H., Nobre, A. C., \& Mesulam, M. M. (2001). Hunger selectively modulates corticolimbic activation to food stimuli in humans. Behavioral neuroscience, 115(2), 493-500. doi:10.1037/0735-7044.115.2.493 
Lancaster, J. L., Rainey, L. H., Summerlin, J. L., Freitas, C. S., Fox, P. T., Evans, a C., Toga, a W., et al. (1997). Automated labeling of the human brain: a preliminary report on the development and evaluation of a forward-transform method. Human brain mapping, 5(4), 238-42. doi:10.1002/(SICI)10970193(1997)5:4<238::AID-HBM6>3.0.CO;2-4

Lancaster, J. L., Tordesillas-Gutiérrez, D., Martinez, M., Salinas, F., Evans, A., Zilles, K., Mazziotta, J. C., et al. (2007). Bias between MNI and Talairach coordinates analyzed using the ICBM-152 brain template. Human brain mapping, 28(11), 1194-205. doi:10.1002/hbm.20345

Lancaster, J. L., Woldorff, M. G., Parsons, L. M., Liotti, M., Freitas, C. S., Rainey, L., Kochunov, P. V, et al. (2000). Automated Talairach atlas labels for functional brain mapping. Human brain mapping, 10(3), 120-31.

Lawrence, N. S., Hinton, E. C., Parkinson, J. a, \& Lawrence, A. D. (2012). Nucleus accumbens response to food cues predicts subsequent snack consumption in women and increased body mass index in those with reduced self-control. NeuroImage, 63(1), 415-422. doi:10.1016/j.neuroimage.2012.06.070

Libet, B., Gleason, C. A., Wright, E. W., \& Pearl, D. K. (1983). Time of conscious intention to act in relation to onset of cerebral activity (readiness-potential). The unconscious initiation of a freely voluntary act. Brain: a journal of neurology, 106 (Pt 3), 623-42.

Mack, A., \& Rock, I. (1998). Inattentional blindness. Cambridge: MIT Press.

Mainz, V., Drüke, B., Boecker, M., Kessel, R., Gauggel, S., \& Forkmann, T. (2012). Influence of cue exposure on inhibitory control and brain activation in patients with alcohol dependence. Frontiers in human neuroscience, 6(May), 92. doi:10.3389/fnhum.2012.00092

Malik, S., McGlone, F., Bedrossian, D., \& Dagher, A. (2008). Ghrelin modulates brain activity in areas that control appetitive behavior. Cell metabolism, 7(5), 400-9. doi:10.1016/j.cmet.2008.03.007

Martens, M. J. I., Born, J. M., Lemmens, S. G. T., Karhunen, L., Heinecke, A., Goebel, R., Adam, T. C., et al. (2013). Increased sensitivity to food cues in the fasted state and decreased inhibitory control in the satiated state in the overweight. The American journal of clinical nutrition, 97(3), 471-9. doi:10.3945/ ajcn.112.044024

Martin, L. E., Holsen, L. M., Chambers, R. J., Bruce, A. S., Brooks, W. M., Zarcone, J. R., Butler, M. G., et al. (2010). Neural mechanisms associated with food motivation in obese and healthy weight adults. Obesity (Silver Spring, Md.), 18(2), 254-60. doi:10.1038/oby.2009.220

Martinez-Mallén, E., Castro-Fornieles, J., Lázaro, L., Moreno, E., Morer, A., Font, E., Julien, J., et al. (2007). Cue exposure in the treatment of resistant adolescent bulimia nervosa. The International journal of eating disorders, 40(7), 596-601. doi:10.1002/eat.20423

McCaffery, J. M., Haley, A. P., Sweet, L. H., Phelan, S., Raynor, H. A., DelParigi, A., Cohen, R., et al. (2009). Differential functional magnetic resonance imaging response to food pictures in successful weightloss maintainers relative to normal-weight and obese controls. The American journal of clinical nutrition, 90(4), 928-34. doi:10.3945/ajcn.2009.27924

McCrory, M. A., Fuss, P. J., McCallum, J. E., Yao, M., Vinken, A. G., Hays, N. P., \& Roberts, S. B. (1999). Dietary variety within food groups: association with energy intake and body fatness in men and women. The American journal of clinical nutrition, 69(3), 440-7. 
Metcalfe, J., \& Mischel, W. (1999). A hot/cool-system analysis of delay of gratification: dynamics of willpower. Psychological review, 106(1), 3-19.

Moore, T., \& Armstrong, K. M. (2003). Selective gating of visual signals by microstimulation of frontal cortex. Nature, 421(6921), 370-3. doi:10.1038/nature01341

Morewedge, C. K., Huh, Y. E., \& Vosgerau, J. (2010). Thought for food: imagined consumption reduces actual consumption. Science (New York, N.Y.), 330(6010), 1530-3. doi:10.1126/science.1195701

Mufson, E. J., \& Mesulam, M. M. (1984). Thalamic connections of the insula in the rhesus monkey and comments on the paralimbic connectivity of the medial pulvinar nucleus. The Journal of comparative neurology, 227(1), 109-20. doi:10.1002/cne.902270112

Muggleton, N. G., Chen, C.-Y., Tzeng, O. J. L., Hung, D. L., \& Juan, C.-H. (2010). Inhibitory control and the frontal eye fields. Journal of cognitive neuroscience, 22(12), 2804-12. doi:10.1162/jocn.2010.21416

Mugler, J. P., \& Brookeman, J. R. (1990). Three-dimensional magnetization-prepared rapid gradient-echo imaging (3D MP RAGE). Magnetic resonance in medicine: official journal of the Society of Magnetic Resonance in Medicine / Society of Magnetic Resonance in Medicine, 15(1), 152-7.

Müller, J., Dettmer, D., \& Macht, M. (2008). The Attitudes to Chocolate Questionnaire: psychometric properties and relationship to dimensions of eating. Appetite, 50(2-3), 499-505. doi:10.1016/j.appet.2007.10.008

Muraven, M., \& Shmueli, D. (2006). The self-control costs of fighting the temptation to drink. Psychology of addictive behaviors: journal of the Society of Psychologists in Addictive Behaviors, 20(2), 154-60. doi:10.1037/0893-164X.20.2.154

Murdaugh, D. L., Cox, J. E., Cook, E. W., \& Weller, R. E. (2012). fMRI reactivity to high-calorie food pictures predicts short- and long-term outcome in a weight-loss program. NeuroImage, 59(3), 2709-21. doi:10.1016/j.neuroimage.2011.10.071

Nisbett, R. E., \& Wilson, T. D. (1977). Telling more than we can know: Verbal reports on mental processes. Psychological Review, 84(3), 231-259. doi:10.1037/0033-295X.84.3.231

Nummenmaa, L., Hirvonen, J., Hannukainen, J. C., Immonen, H., Lindroos, M. M., Salminen, P., \& Nuutila, P. (2012). Dorsal striatum and its limbic connectivity mediate abnormal anticipatory reward processing in obesity. PloS one, 7(2), e31089. doi:10.1371/journal.pone.0031089

Pavlov, I. P. (1927). Conditioned reflexes. London: Oxford University Press.

Pelchat, M. L. (1997). Food cravings in young and elderly adults. Appetite, 28(2), 103-13. doi:10.1006/ appe.1996.0063

Pessoa, L., \& Adolphs, R. (2010). Emotion processing and the amygdala: from a "low road" to "many roads" of evaluating biological significance. Nature reviews. Neuroscience, 11(11), 773-83. doi:10.1038/ nrn2920

Pessoa, L., Padmala, S., \& Morland, T. (2005). Fate of unattended fearful faces in the amygdala is determined by both attentional resources and cognitive modulation. NeuroImage, 28(1), 249-55. doi:10.1016/j. neuroimage.2005.05.048

Pizam, A. (2013). The prevalence of obesity among foodservice employees. International Journal of Hospitality Management, 32, 1. doi:10.1016/j.ijhm.2012.09.002 
Pliner, P., Herman, C. P., \& Polivy, J. (1990). Palatability as a determinant of eating: Finickiness as a function of taste, hunger, and the prospect of good food. In E. D. Capaldi \& T. L. Powley (Eds.), Taste, experience, and feeding. (pp. 210-226). Washington, DC, US: American Psychological Association.

PST (1996). E-Prime (Version 2.0.8.90) [Computer software]. Pittsburgh, PA, USA: Psychology Software Tools Inc. Retrieved from http://www.pstnet.com.

Remick, A. K., Polivy, J., \& Pliner, P. (2009). Internal and external moderators of the effect of variety on food intake. Psychological bulletin, 135(3), 434-51. doi:10.1037/a0015327

Roefs, A., Huijding, J., Smulders, F. T. Y., MacLeod, C. M., De Jong, P. J., Wiers, R. W., \& Jansen, A. (2011). Implicit measures of association in psychopathology research. Psychological bulletin, 137(1), 149-93. doi:10.1037/a0021729

Roefs, A., Jansen, A., Dijk, F., Hofstra, L., Martijn, C., Van Breukelen, G., \& Nederkoorn, C. (2012). The Role of Depressive Symptoms in the Relation between Dieting Motivation and Weight Change. Journal of Social and Clinical Psychology, 31(9), 1007-1021. doi:10.1521/jscp.2012.31.9.1007

Rogers, P. J., \& Smit, H. J. (2000). Food craving and food "addiction": a critical review of the evidence from a biopsychosocial perspective. Pharmacology, biochemistry, and behavior, 66(1), 3-14.

Rolls, B. J. (2000). The role of energy density in the overconsumption of fat. The Journal of nutrition, 130(2S Suppl), 268S-271S.

Rolls, E. T. (2011). Taste, olfactory and food texture reward processing in the brain and obesity. International Journal of Obesity, 35(4), 550-61. doi:10.1038/ijo.2010.155

Rosen, B. R., \& Savoy, R. L. (2012). fMRI at 20: has it changed the world? NeuroImage, 62(2), 1316-24. doi:10.1016/j.neuroimage.2012.03.004

Rotenberg, K. J., Lancaster, C., Marsden, J., Pryce, S., Williams, J., \& Lattimore, P. (2005). Effects of priming thoughts about control on anxiety and food intake as moderated by dietary restraint. Appetite, 44(2), 235-41. doi:10.1016/j.appet.2004.09.001

Rothemund, Y., Preuschhof, C., Bohner, G., Bauknecht, H.-C., Klingebiel, R., Flor, H., \& Klapp, B. F. (2007). Differential activation of the dorsal striatum by high-calorie visual food stimuli in obese individuals. NeuroImage, 37(2), 410-21. doi:10.1016/j.neuroimage.2007.05.008

Rozin, P., Dow, S., Moscovitch, M., \& Rajaram, S. (1998). What Causes Humans to Begin and End a Meal? A Role for Memory for What Has Been Eaten, as Evidenced by a Study of Multiple Meal Eating in Amnesic Patients. Psychological Science, 9(5), 392-396. doi:10.1111/1467-9280.00073

Rutters, F., Nieuwenhuizen, A. G., Lemmens, S. G. T., Born, J. M., \& Westerterp-Plantenga, M. S. (2009). Acute stress-related changes in eating in the absence of hunger. Obesity (Silver Spring, Md.), 17(1), 72-7. doi:10.1038/oby.2008.493

Sassi, F., Cecchini, M., \& Devaux, M. (2010). Obesity and the economics of prevention: Fit not fat. (F. Sassi, Ed.). Paris, France: OECD Publishing.

Sassi, F., \& Devaux, M. (2012). OBESITY UPDATE 2012 (pp. 1-7). Paris, France: OECD Publishing.

Scagliusi, F. B., Polacow, V. O., Cordás, T. A., Coelho, D., Alvarenga, M., Philippi, S. T., \& Lancha, A. H. (2005). Test-retest reliability and discriminant validity of the Restraint Scale translated into Portuguese. Eating behaviors, 6(1), 85-93. doi:10.1016/j.eatbeh.2004.06.001 
Scharmüller, W., Übel, S., Ebner, F., \& Schienle, A. (2012). Appetite regulation during food cue exposure: a comparison of normal-weight and obese women. Neuroscience letters, 518(2), 106-10. doi:10.1016/j. neulet.2012.04.063

Schienle, A., Schäfer, A., Hermann, A., \& Vaitl, D. (2009). Binge-eating disorder: reward sensitivity and brain activation to images of food. Biological psychiatry, 65(8), 654-61. doi:10.1016/j.biopsych.2008.09.028

Schloegl, H., Percik, R., Horstmann, A., Villringer, A., \& Stumvoll, M. (2011). Peptide hormones regulating appetite--focus on neuroimaging studies in humans. Diabetes/metabolism research and reviews, 27(2), 104-12. doi:10.1002/dmrr.1154

Schoeller, D. A. (2009). The energy balance equation: looking back and looking forward are two very different views. Nutrition reviews, 67(5), 249-54. doi:10.1111/j.1753-4887.2009.00197.x

Schubert, D. S. (1975). Increase of personality response consistency by prior response. Journal of clinical psychology, 31(4), 651-8.

Schubert, D. S., \& Fiske, D. W. (1973). Increase of Item Response Consistency By Prior Item Response. Educational and Psychological Measurement, 33(1), 113-121. doi:10.1177/001316447303300112

Schwarz, N., \& Oyserman, D. (2001). Asking questions about behavior: cognition, communication, and questionnaire construction. The American Journal of Evaluation, 22(2), 127-160. doi:10.1016/S10982140(01)00133-3

Serences, J. T. (2004). A comparison of methods for characterizing the event-related BOLD timeseries in rapid fMRI. NeuroImage, 21(4), 1690-700. doi:10.1016/j.neuroimage.2003.12.021

Siep, N., Roefs, A., Roebroeck, A., Havermans, R., Bonte, M., \& Jansen, A. (2012). Fighting food temptations: the modulating effects of short-term cognitive reappraisal, suppression and up-regulation on mesocorticolimbic activity related to appetitive motivation. NeuroImage, 60(1), 213-20. doi:10.1016/j.neuroimage.2011.12.067

Siep, N., Roefs, A., Roebroeck, A., Havermans, R., Bonte, M. L., \& Jansen, A. (2009). Hunger is the best spice: an fMRI study of the effects of attention, hunger and calorie content on food reward processing in the amygdala and orbitofrontal cortex. Behavioural brain research, 198(1), 149-58. doi:10.1016/j. bbr.2008.10.035

Simmons, W. K., Martin, A., \& Barsalou, L. W. (2005). Pictures of appetizing foods activate gustatory cortices for taste and reward. Cerebral Cortex, 15(10), 1602-8. doi:10.1093/cercor/bhi038

Small, D. M. (2009). Individual differences in the neurophysiology of reward and the obesity epidemic. International Journal of Obesity, 33 Suppl 2, S44-8. doi:10.1038/ijo.2009.71

Small, D. M., Gregory, M. D., Mak, Y. E., Gitelman, D., Mesulam, M. M., \& Parrish, T. (2003). Dissociation of neural representation of intensity and affective valuation in human gustation. Neuron, 39(4), 701-11.

Small, D. M., Jones-Gotman, M., \& Dagher, A. (2003). Feeding-induced dopamine release in dorsal striatum correlates with meal pleasantness ratings in healthy human volunteers. NeuroImage, 19(4), 1709-15. doi:10.1016/S1053-8119(03)00253-2

Small, D. M., Zatorre, R. J., Dagher, A., Evans, A. C., \& Jones-Gotman, M. (2001). Changes in brain activity related to eating chocolate: from pleasure to aversion. Brain: a journal of neurology, 124(Pt 9), 1720-33. 
Smeets, P., De Graaf, C., Stafleu, A., Van Osch, M. J. P., Nievelstein, R. a J., \& Van der Grond, J. (2006). Effect of satiety on brain activation during chocolate tasting in men and women. The American journal of clinical nutrition, 83(6), 1297-305.

Sobik, L., Hutchison, K., \& Craighead, L. (2005). Cue-elicited craving for food: a fresh approach to the study of binge eating. Appetite, 44(3), 253-61. doi:10.1016/j.appet.2004.12.001

Soon, C. S., Brass, M., Heinze, H.-J., \& Haynes, J.-D. (2008). Unconscious determinants of free decisions in the human brain. Nature neuroscience, 11(5), 543-5. doi:10.1038/nn.2112

Stice, E., Spoor, S., Bohon, C., Veldhuizen, M. G., \& Small, D. M. (2008). Relation of reward from food intake and anticipated food intake to obesity: a functional magnetic resonance imaging study. Journal of abnormal psychology, 117(4), 924-35. doi:10.1037/a0013600

Stice, E., Yokum, S., Bohon, C., Marti, N., \& Smolen, A. (2010). Reward circuitry responsivity to food predicts future increases in body mass: moderating effects of DRD2 and DRD4. NeuroImage, 50(4), 1618-25. doi:10.1016/j.neuroimage.2010.01.081

Stice, E., Yokum, S., Burger, K. S., Epstein, L. H., \& Small, D. M. (2011). Youth at risk for obesity show greater activation of striatal and somatosensory regions to food. The Journal of neuroscience: the official journal of the Society for Neuroscience, 31(12), 4360-6. doi:10.1523/JNEUROSCI.6604-10.2011

Stoeckel, L. E., Kim, J., Weller, R. E., Cox, J. E., Cook, E. W., \& Horwitz, B. (2009). Effective connectivity of a reward network in obese women. Brain research bulletin, 79(6), 388-95. doi:10.1016/j.brainresbull.2009.05.016

Stoeckel, L. E., Murdaugh, D. L., Cox, J. E., Cook, E. W., \& Weller, R. E. (2012). Greater impulsivity is associated with decreased brain activation in obese women during a delay discounting task. Brain imaging and behavior. doi:10.1007/s11682-012-9201-4

Stoeckel, L. E., Weller, R. E., Cook, E. W., Twieg, D. B., Knowlton, R. C., \& Cox, J. E. (2008). Widespread reward-system activation in obese women in response to pictures of high-calorie foods. NeuroImage, 41(2), 636-47. doi:10.1016/j.neuroimage.2008.02.031

St-Onge, M.-P., Sy, M., Heymsfield, S. B., \& Hirsch, J. (2005). Human cortical specialization for food: a functional magnetic resonance imaging investigation. The Journal of nutrition, 135(5), 1014-8.

Stubbs, R. J., Hughes, D., Johnstone, A., Rowley, E., Reid, C., Elia, M., Stratton, R., et al. (2000). The use of visual analogue scales to assess motivation to eat in human subjects: a review of their reliability and validity with an evaluation of new hand-held computerized systems for temporal tracking of appetite ratings. The British journal of nutrition, 84(4), 405-15. doi:10.1017/S0007114500001719

Swinburn, B. a, Sacks, G., Hall, K. D., McPherson, K., Finegood, D. T., Moodie, M. L., \& Gortmaker, S. L. (2011). The global obesity pandemic: shaped by global drivers and local environments. Lancet, 378(9793), 804-14. doi:10.1016/S0140-6736(11)60813-1

Talairach, J., \& Tournoux, P. (1988). Co-Planar Stereotaxic Atlas of the Human Brain: 3-Dimensional Proportional System - an Approach to Cerebral Imaging. New York: Thieme Medical Publishers.

Tang, D. W., Fellows, L. K., Small, D. M., \& Dagher, A. (2012). Food and drug cues activate similar brain regions: a meta-analysis of functional MRI studies. Physiology \& behavior, 106(3), 317-24. doi:10.1016/j.physbeh.2012.03.009 
Tiggemann, M., \& Kemps, E. (2005). The phenomenology of food cravings: the role of mental imagery. Appetite, 45(3), 305-13. doi:10.1016/j.appet.2005.06.004

Toro, J., Cervera, M., Feliu, M. H., Garriga, N., Jou, M., Martinez, E., \& Toro, E. (2003). Cue exposure in the treatment of resistant bulimia nervosa. The International journal of eating disorders, 34(2), 227-34. doi:10.1002/eat.10186

Uher, R., Treasure, J., Heining, M., Brammer, M. J., \& Campbell, I. C. (2006). Cerebral processing of foodrelated stimuli: effects of fasting and gender. Behavioural brain research, 169(1), 111-9. doi:10.1016/j. bbr.2005.12.008

Van der Laan, L. N., De Ridder, D., Viergever, M., \& Smeets, P. (2011). The first taste is always with the eyes: a meta-analysis on the neural correlates of processing visual food cues. NeuroImage, 55(1), 296-303. doi:10.1016/j.neuroimage.2010.11.055

Van Gucht, D., Vansteenwegen, D., Beckers, T., Hermans, D., Baeyens, F., \& Van den Bergh, O. (2008). Repeated cue exposure effects on subjective and physiological indices of chocolate craving. Appetite, 50(1), 19-24. doi:10.1016/j.appet.2007.05.003

Van Gucht, D., Vansteenwegen, D., Beckers, T., \& Van den Bergh, O. (2008). Return of experimentally induced chocolate craving after extinction in a different context: divergence between craving for and expecting to eat chocolate. Behaviour research and therapy, 46(3), 375-91. doi:10.1016/j. brat.2008.01.003

Van Strien, T., Herman, C. P., Engels, R. C. M. E., Larsen, J. K., \& Van Leeuwe, J. F. J. (2007). Construct validation of the Restraint Scale in normal-weight and overweight females. Appetite, 49(1), 109-21. doi:10.1016/j.appet.2007.01.003

Veenstra, E. M., De Jong, P. J., Koster, E. H. W., \& Roefs, A. (2010). Attentional avoidance of high-fat food in unsuccessful dieters. Journal of behavior therapy and experimental psychiatry, 41(3), 282-8. doi:10.1016/j.jbtep.2010.02.006

Viskaal-van Dongen, M., Kok, F. J., \& De Graaf, C. (2011). Eating rate of commonly consumed foods promotes food and energy intake. Appetite, 56(1), 25-31. doi:10.1016/j.appet.2010.11.141

Vohs, K. D., \& Heatherton, T. F. (2000). Self-regulatory failure: a resource-depletion approach. Psychological science: a journal of the American Psychological Society / APS, 11(3), 249-54.

Volkow, N. D., Wang, G.-J., Wang, G., Telang, F., Fowler, J. S., Goldstein, R. Z., Alia-klein, N., et al. (2009). Inverse association between BMI and prefrontal metabolic activity in healthy adults. Obesity (Silver Spring, Md.), 17(1), 60-5. doi:10.1038/oby.2008.469

Vollstädt-Klein, S., Loeber, S., Kirsch, M., Bach, P., Richter, A., Bühler, M., Von der Goltz, C., et al. (2011). Effects of cue-exposure treatment on neural cue reactivity in alcohol dependence: a randomized trial. Biological psychiatry, 69(11), 1060-6. doi:10.1016/j.biopsych.2010.12.016

Vuilleumier, P., Richardson, M. P., Armony, J. L., Driver, J., \& Dolan, R. J. (2004). Distant influences of amygdala lesion on visual cortical activation during emotional face processing. Nature neuroscience, 7(11), 1271-8. doi:10.1038/nn1341

Vuilleumier, P., Schwartz, S., Duhoux, S., Dolan, R. J., \& Driver, J. (2005). Selective attention modulates neural substrates of repetition priming and "implicit" visual memory: suppressions and enhancements revealed by FMRI. Journal of cognitive neuroscience, 17(8), 1245-60. doi:10.1162/0898929055002409 
Wallner-Liebmann, S., Koschutnig, K., Reishofer, G., Sorantin, E., Blaschitz, B., Kruschitz, R., Unterrainer, H. F., et al. (2010). Insulin and hippocampus activation in response to images of high-calorie food in normal weight and obese adolescents. Obesity (Silver Spring, Md.), 18(8), 1552-7. doi:10.1038/ oby. 2010.26

Walther, K., Birdsill, A. C., Glisky, E. L., \& Ryan, L. (2009). Structural brain differences and cognitive functioning related to body mass index in older females. Human brain mapping, 1064, 1052-1064. doi:10.1002/hbm.20916

Wang, G.-J., Volkow, N. D., Telang, F., Jayne, M., Ma, Y., Pradhan, K., Zhu, W., et al. (2009). Evidence of gender differences in the ability to inhibit brain activation elicited by food stimulation. Proceedings of the National Academy of Sciences of the United States of America, 106(4), 1249-54. doi:10.1073/pnas.0807423106

Wansink, B., Painter, J. E., \& North, J. (2005). Bottomless bowls: why visual cues of portion size may influence intake. Obesity research, 13(1), 93-100. doi:10.1038/oby.2005.12

Watson, D., Clark, L. A., \& Tellegen, A. (1988). Development and validation of brief measures of positive and negative affect: the PANAS scales. Journal of personality and social psychology, 54(6), 1063-70.

Weierich, M. R., Wright, C. I., Negreira, A., Dickerson, B. C., \& Barrett, L. F. (2010). Novelty as a dimension in the affective brain. NeuroImage, 49(3), 2871-8. doi:10.1016/j.neuroimage.2009.09.047

Weiskopf, N., Hutton, C., Josephs, O., Turner, R., \& Deichmann, R. (2007). Optimized EPI for fMRI studies of the orbitofrontal cortex: compensation of susceptibility-induced gradients in the readout direction. Magma (New York, N.Y.), 20(1), 39-49. doi:10.1007/s10334-006-0067-6

Werthmann, J., Roefs, A., Nederkoorn, C., Mogg, K., Bradley, B. P., \& Jansen, A. (2011). Can(not) take my eyes off it: Attention bias for food in overweight participants. Health psychology: official journal of the Division of Health Psychology, American Psychological Association. doi:10.1037/a0024291

Williamson, D. A., Martin, C. K., York-Crowe, E., Anton, S. D., Redman, L. M., Han, H., \& Ravussin, E. (2007). Measurement of dietary restraint: validity tests of four questionnaires. Appetite, 48(2), 183-92. doi:10.1016/j.appet.2006.08.066

World Health Organization. (2007). The challenge of obesity in the WHO European Region and the strategies for response: summary. (F. Branca, H. Nikogosian, \& T. Lobstein, Eds.). Copenhagen: World Health Organization.

Yokum, S., Ng, J., \& Stice, E. (2011a). Relation of regional gray and white matter volumes to current BMI and future increases in BMI: a prospective MRI study. International Journal of Obesity, 1-9. doi:10.1038/ijo.2011.175

Yokum, S., Ng, J., \& Stice, E. (2011b). Attentional Bias to Food Images Associated With Elevated Weight and Future Weight Gain: An fMRI Study. Obesity (Silver Spring, Md.), 19(9), 1775-1783. doi:10.1038/ oby. 2011.168

Zheng, H., Lenard, N. R., Shin, A. C., \& Berthoud, H.-R. (2009). Appetite control and energy balance regulation in the modern world: reward-driven brain overrides repletion signals. International Journal of Obesity, 33 Suppl 2, S8-13. doi:10.1038/ijo.2009.65

Zweynert, S., Pade, J. P., Wüstenberg, T., Sterzer, P., Walter, H., Seidenbecher, C. I., Richardson-Klavehn, A., et al. (2011). Motivational salience modulates hippocampal repetition suppression and functional connectivity in humans. Frontiers in human neuroscience, 5(November), 144. doi:10.3389/ fnhum.2011.00144 



\section{DANKWOORD}

Dit proefschrift kon slechts tot stand komen met de hulp en medewerking van heel veel mensen. Daarom op deze plek een woord van dank aan allen die hiertoe hebben bijgedragen. Met name wil ik de volgende personen noemen.

Allereerst een heel groot woord van dank aan mijn promotor, Anita Jansen, en mijn copromotor en supervisor, Anne Roefs, voor het in mij gestelde vertrouwen om mij deze promotieplek überhaupt te geven, alhoewel ik een heel andere achtergrond had. Bovendien had ik zonder jullie hulp in de laatste maanden dit proefschrift niet zo snel kunnen afmaken. Vooral de snelle feedbackrondes door Anne in de laatste weken voor de deadline waren onmisbaar en hebben mij bij het schrijven enorm ondersteund. Meestal was de feedback op de nieuwe versies van de hoofdstukken weer sneller terug dan ik de commentaren kon inwerken $(-)$. Ook Anita zette flink vaart achter het geven van feedback. Dank jullie wel voor het geven van zo'n hoge prioriteit aan het nakijken van mijn proefschrift! Omdat Anne en Anita de eersten in Maastricht waren die van mijn zwangerschap wisten, waren onze meetings de laatste maanden heel persoonlijk, wat ik erg leuk vond. Heel veel dank dus ook voor alle zwangerschapstips! :;

Ook bedank ik Alard, mijn tweede co-promotor, voor zijn hulp bij het ontwerpen van de paradigma's en de soms moeilijke keuze wat betreft de meest geschikte analyse van de resultaten.

De co-auteurs van mijn papers, waartoe naast mijn (co-)promotoren ook Nicolette Siep en Remco Havermans behoorden, dank ik voor hun ideeën bij het opzetten van de studies en voor hun feedback op de manuscripten van de papers. Afgezien van co-auteur was Nicolette zowel mijn kamergenootje alsook mijn voorganger in het doen van fMRI-studies binnen de eetgroep. Hierdoor heeft ze vooral in het begin heel veel vragen van mijn kant moeten verduren. Heel erg bedankt voor je geduld en voor het terugvinden van de rode draad, als ik me bij de analyses weer eens te zeer door veelvoudige details liet afleiden! 
I would like to thank the members of the promotion committee, Prof. dr. B. Jansma, Prof. dr. C. de Graaf, Dr. S. Higgs, Dr. R. Ruiter, and Prof. dr. A. Schols, for the time and effort they have spent reading and evaluating this thesis and for their questions during the defense.

I thank all participants in my experiments for being very patient and for conscientiously following the instructions. My fMRI-backup-buddies made it possible to scan: Tobias, Christine, Charlotte, Luciana, Fritz, Judith, Christianne, Eva, Silke, Zoe and Linda. Thanks for your help!

Die Analysen der fMRI-Ergebnisse waren nicht immer ganz einfach. Hierbei war Armin Heinecke mir sehr behilflich, wofür ich ihm herzlich danke. Ook de jongens (zoals ik ze noem) van de afdeling instrumentatie waren altijd heel behulpzaam en attent, waarbij ik vooral Charlie Bonnemayer apart zou willen noemen als onmisbare hulp bij het programmeren van het eerste taakje in E-Prime. Het is jammer dat de collega's bij mijn volgende baan het Bocholtzer Plat waarschijnlijk helemáál niet zullen verstaan ... ;-). Bij de doorvoering van de fMRI-experimenten heb ik veel hulp gehad van Sven en en Lotty. Bedankt!

Beste collega's van CPS en van de eetgroep (het A-team!) in het bijzonder: bedankt voor alle inspiratie en collegiale uitwisseling, wekelijkse eet-meetings, voor de gezellige EPPcursussen, lunchpauzes, tuinfeest-avonden en vla-momenten (met dank aan Angela!). Onze secretaresses Jessie, Truus \& Lindy, bedankt voor alle ondersteuning en alle ongecompliceerde en snelle hulp! Johanna, schade dass wir nun nicht mehr gemeinsam zur Arbeit fahren können, mit Dir war es immer sehr gemütlich im Auto! UM-girls, zullen we voor eind september nog een keertje uitgaan? Ik ken nu een adresje in Düsseldorf;-). $\mathrm{Nu}$ ik wegga bij de UM kan ik helaas ook niet meer gaan lunchen met collega's van andere afdelingen. Ewout, Joost, Judith, Sanae, Tobias en Luciana, het was altijd heel gezellig!

Aan mijn vroegere en huidige kamergenootjes, ik zal jullie missen! Charlotte, je was mijn eerste collegaatje op de UM en ik werd uiteindelijk je paranimf! Dat was een heel leuke en eervolle ervaring. Eva (W.), ik stond er versteld van hoe snel jij Nederlands kon spreken! Janneke, zonder jou als scout had ik nooit gedurfd om in zo'n vroeg stadium van mijn studie op dit project te solliciteren. Bedankt! Katrijn, om jouw verhalen over je kat Uschi heb ik altijd erg moeten lachen. Ramona, als ik ooit nog eens zo'n lieve collega tegenkom dan heb ik het wel héél erg getroffen. Nicolette, afgezien van alle goede fMRIraad, bedankt voor de leuke gesprekken en de zwangerschapstips! Dankzij jou neem ik nu visolie-capsules $;$. Suzanne, het is alsof we al jaren collega's zijn, terwijl je er nog niet zo heel lang bent. Jammer dat ik binnenkort moet gaan! Eva (A.), you have been a lovely colleague and I wish you good luck with your little girl! :; 
Bei meinen Freunden und Bekannten möchte ich mich für ihre Unterstützung und Geduld bedanken und dafür, dass sie oft den Kontakt mit mir gehalten haben, obwohl ich selber in Sachen Freundschaftspflege manchmal eine treuelose Tomate bin und häufig erst nach großer Verzögerung zurückrufe oder zurückmaile. Ich habe trotzdem an Euch gedacht und Euch nicht vergessen!!! Insbesondere meinen Freunden Giusy, Diep, Mahmoud, Olaf und Sophie, möchte ich danken für ihre seelische (oft leider nur telefonische) Unterstützung, en dat geldt natuurlijk ook en zeker niet in de laatste plaats voor Iris, Birgit en Esther. Dear friends overseas, thank you for staying in touch even though it is difficult for me to reply in time ... I'm sorry for that, and please believe me when I say that I didn't forget you! Chers amis en France, il faut que nous nous rencontrons rapidement... il y a longtemps.

Lieve paranimfjes, Iris en Jessica, ik ben trots dat jullie op mijn promotiedag achter me staan, ondanks de stress die jullie zelf al genoeg hebben. Jullie zijn allebei op jullie eigen manier ontzettend open en optimistisch en ik hoop dat ik daar ook in de toekomst nog vaak iets van mag leren. En Iris, die meteorietinslag komt misschien pas op ons $73^{\mathrm{e}} \ldots$ tot die tijd kunnen we nog genieten ;-)

Lieve papa en mama: hoe ouder ik word, hoe meer ik besef dat het niet vanzelfsprekend is dat ik bij jullie niet hoef na te denken over wat ik wel en niet kan zeggen. Jullie denken steeds constructief en in alle vertrouwen na over mogelijke oplossingen voor problemen die soms zó uit een soapserie zouden kunnen komen. Zo'n geluk heeft niet iedereen. Dat geldt ook voor jou Essie, mijn kleine grote zus, en voor jou Arndt. Danke dass Du immer ohne Murren hilfst, auch wenn Umzüge Dir gar keinen Spaß machen ;-). Philip en Daniel, ik verheug me erop dat ik na de promotie weer wat meer tijd heb en we weer af en toe leuke dingen samen kunnen gaan doen (ook al zit een klimparcours er met die dikke buik nu even niet in, ben ik bang).

Bovendien nog een grote loftrompet voor mama en Rob. Mama heeft me de laatste maanden bijna dagelijks met maaltijden verzorgd en de was gedaan, zodat ik me helemaal kon concentreren op mijn proefschrift. In de weekenden nam Rob dat van haar over, deed boodschappen en ruimde ook nog het aanrecht op. Wat had en zou ik zonder jullie moeten doen?

Lieve Rob, dankjewel voor je motivatie in de vorm van meerdere dozen Leonidas-bonbons en kadootjes, die ik pas mocht openmaken naarmate het proefschrift vorderde. Je ziet het, het heeft geholpen (:)! Natuurlijk is dat niet alles wat je voor me betekent, maar dat schrijf ik liever in een persoonlijke Widmung in jouw exemplaar van dit boekje (en spätestens im Oktober motte ver ooch jit doë aan os taalgebruik...;-)

Dag klein mokkeltje, je hebt mama hard laten werken de laatste tijd ... maar gelukkig heeft het zich geloond en heb ik nu eindelijk tijd om me te verheugen op jouw komst. 



\section{Curriculum Vitae}

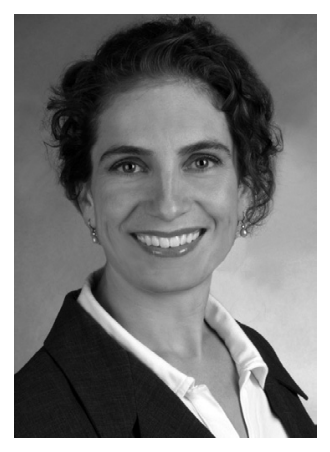

Astrid Frankort was born on February 20, 1975 in Waalre, The Netherlands. In 1992 she graduated cum laude from secondary school (Bernardinuscollege in Heerlen). Her first studies of electrical engineering at RWTH Aachen University, with a major in telecommunication engineering and electives in biomedical engineering, were successfully completed in 2000. She spent several months at Yamagata University, Japan, for a student research project in biomedical engineering. After her graduation, she continued to work at the Institute of Communication Systems and Data Processing of RWTH Aachen University as a scientific assistant. In 2001, she moved to Singapore and worked there for a couple of months as a technical consultant at Siemens Information and Communication Mobile. Later in the same year, she enrolled in an MBA management program at the Collège des Ingénieurs in Paris, France, which was combined with an internship at Siemens Medical Solutions in Erlangen, Germany. After obtaining her MBA in 2002, she joined her father's engineering company in Heerlen as an executive manager and head of sales. In 2007 she started her studies of psychology at Maastricht University and entered the Master in Cognitive Neuroscience in 2008, which she completed cum laude in August 2009. During her studies, in January 2009, she started her PhD position in the group of Eating Disorders at Maastricht University, under the supervision of Prof. dr. Anita Jansen and the co-supervision of Dr. Anne Roefs and Dr. Alard Roebroeck. The results of her $\mathrm{PhD}$ research are described in this thesis. 


\section{Publications}

Frankort, A., Roefs, A., Siep, N., Roebroeck, A., Havermans, R., \& Jansen, A. (n.d.). Reward or inhibition when seeing palatable food? It all depends on your weight and your focus of attention. Manuscript in preparation.

Frankort, A., Roefs, A., Siep, N., Roebroeck, A., Havermans, R., \& Jansen, A. (n.d.). Neural predictors of chocolate intake following chocolate exposure. Manuscript in revision at NeuroImage.

Frankort, A., Roefs, A., Siep, N., Roebroeck, A., Havermans, R., \& Jansen, A. (in press). The Craving Stops Before You Feel It: Neural Correlates of Chocolate Craving During Cue Exposure with Response Prevention. Cerebral Cortex. doi:10.1093/cercor/bht016

Frankort, A., Roefs, A., Siep, N., Roebroeck, A., Havermans, R., \& Jansen, A. (2012). Reward activity in satiated overweight women is decreased during unbiased viewing but increased when imagining taste: an event-related fMRI study. International journal of obesity, 36(5), 627-37. doi:10.1038/ijo.2011.213

Havermans, R. C., Heijens, T., Frankort, A., \& Jansen, A. (2010). Exercise-induced flavour aversion learning in humans. Appetite, 55(1), 170-170. doi:10.1016/j.appet.2010.05.071.

Hoelper, C., Frankort, A., Erdmann, C., \& Vary, P. (2002). A novel voiced / unvoiced / silence classification scheme for offline speech coding. Proceedings of European Signal Processing Conference (EUSIPCO) 2002. Toulouse. 


\section{Presentations}

Frankort, A., Roefs, A., Siep, N., Roebroeck, A., Havermans, R., \& Jansen, A. (2013, planned). Neural correlates of chocolate craving during cue exposure with response prevention. Poster presentation at the "19th Annual Meeting of the Organization for $\mathrm{Hu}$ man Brain Mapping" in Seattle, USA.

Frankort, A., Roefs, A., Siep, N., Roebroeck, A., Havermans, R., \& Jansen, A. (2012). Neural Correlates of Craving during Cue Exposure with Response Prevention (CERP) with Chocolate. Invited oral presentation at the "10th Dutch Endo-Neuro-Psycho Meeting", Lunteren, The Netherlands.

Frankort, A., Roefs, A., Siep, N., Roebroeck, A., Havermans, R., \& Jansen, A. (2012). Neural correlates of craving during cue exposure with response prevention (CERP) with chocolate. Poster presented at the "1st Conference of the European Society for Cognitive and Affective Neuroscience" in Marseille, France.

Frankort, A., Roefs, A., Siep, N., Roebroeck, A., Havermans, R., \& Jansen, A. (2012). Reward activity in satiated overweight women is decreased during unbiased viewing but increased when imagining taste: an event-related fMRI study. Poster presented at the "1st Conference of the European Society for Cognitive and Affective Neuroscience" in Marseille, France.

Frankort, A., Roefs, A., Siep, N., Roebroeck, A., Havermans, R., \& Jansen, A. (2011). Reward activity in satiated overweight people is decreased during unbiased viewing, but increased during explicit taste evaluation. Invited oral presentation at the "9th Dutch Endo-Neuro-Psycho Meeting", Lunteren, The Netherlands.

Frankort, A., Roefs, A., Siep, N., Roebroeck, A., Havermans, R., \& Jansen, A. (2010). Food reward processing in overweight and healthy-weight participants during unbiased viewing and taste evaluation. Poster presented at the Expert Meeting "Experimental Research into the Psychopathology of Obesity" of the Royal Netherlands Academy of Arts and Sciences (KNAW) in Amsterdam, The Netherlands.

Frankort, A., Roefs, A., Siep, N., Roebroeck, A., Havermans, R., \& Jansen, A. (2010). Food reward processing in overweight and healthy-weight participants during unbiased viewing and taste evaluation. Poster presented at the " 34 th Annual Meeting of the British Feeding and Drinking Group" in Maastricht, The Netherlands. 
